\author{
UNIVERSIDADE DE SÃO PAULO \\ FACULDADE DE ODONTOLOGIA DE BAURU
}

VINICIUS LARANJEIRA BARBOSA DA SILVA

Long-term stability of surgical-orthodontic open bite treatment:

Le Fort I versus 4-piece segmental Le Fort I osteotomy

Estabilidade em longo prazo do tratamento cirúrgico da mordida aberta anterior por osteotomia tipo Le Fort I com e sem segmentação maxilar

BAURU 

Long-term stability of surgical-orthodontic open bite treatment:

Le Fort I versus 4-piece segmental Le Fort I osteotomy

Estabilidade em longo prazo do tratamento cirúrgico da mordida aberta anterior por osteotomia tipo Le Fort I com e sem segmentação maxilar

Tese constituída por artigos apresentada a Faculdade de Odontologia de Bauru da Universidade de São Paulo para obtenção do título de Doutor em Ciências no Programa de Ciências Odontológicas Aplicadas, na área de concentração Ortodontia.

Orientador: Prof. Dr. Guilherme Janson

Versão Corrigida

BAURU 


\begin{tabular}{|l|}
\hline Silva, Vinicius Laranjeira Barbosa da Silva \\
Si38L $\quad$ Long-term stability of surgical-orthodontic open \\
bite treatment: Le Fort I versus 4-piece segmental \\
Le Fort I osteotomy/ Vinicius Laranjeira Barbosa \\
da Silva. -- Bauru, 2018. \\
111p. : il. ; $31 \mathrm{~cm}$. \\
Tese. (Doutorado) -- Faculdade de Odontologia \\
de Bauru. Universidade de São Paulo. \\
Orientador: Prof. Dr. Guilherme Janson
\end{tabular}

Nota: A versão original desta tese encontra-se disponível no Serviço de Biblioteca e Documentação da Faculdade de Odontologia de Bauru - FOB/USP.

Autorizo, exclusivamente, para fins acadêmicos e científicos, a reprodução total ou parcial desta dissertação, por processos fotocopiadores e outros meios eletrônicos.

Assinatura:

Data: $23 / 01 / 2018$

Comitê de Ética da FOB-USP Protocolo no: 71636716.5 .0000 .5417 Data: $23 / 01 / 2018$ 
FOLHA DE APROVAÇÃO 



\section{DEDICATÓRIA}

Dedico este trabalho:

Aos meus amores, Eliane e Laura, pois todo o sacrifício que fizemos e a compreensão que tiveram por minha ausência nessa jornada em busca do conhecimento, nos tornaram mais fortes e unidos, ampliando infinitamente nossa capacidade de sonhar e realizar! 



\section{AGRADECIMENTOS ESPECIAIS}

> Agradeço a todos os professores por terem contribuído para minha formação, em especial, à minha eterna Professora e Mãe, Maria Inês Laranjeira, a qual está sempre me orientando e inspirando com seu conhecimento, tenacidade e dedicação ao exercer essas nobres funções;

Ao meu Pai, Ruy, e meus irmãos, Gustavo e Henrique, pelo carinho, paciência, suporte e incentivo constantes. 



\section{AGRADECIMENTOS}

$>$ Ao meu orientador, Prof. Dr. Guilherme Janson, e a todos os professores da Disciplina de Ortodontia da FOB-USP, pela orientação e ensinamentos ao longo desses mais de 5 anos de convivência;

> A todos meus colegas de Doutorado: Angie, Arthur, Bruna, Bruno, Camilla, Karine, Louise, Mayara, Melissa, Murilo, Rodrigo, Sérgio e Waleska.

Ao meu amigo Arón, que representa todas as outras turmas de especialização, mestrado e doutorado com as quais convivi na FOB-USP.

Aos funcionários da FOB-USP: Vera, Cléo, Sérgio e Wagner pela excelente convivência nessa última meia década;

Ao amigo Daniel pelo suporte técnico e humorístico durante esses anos;

Ao Dr. Francisco Ajalmar Maia por permitir a utilização de seu arquivo de imagens;

Aos cirurgiões Buco-Maxilo-Faciais: Dr. Eduardo Sant'Ana, Dr. Roberto Macoto, Dr. João Roberto Gonçalves e Dr. Renato Maia pela inestimável contribuição ao, gentilmente, compartilharem suas amostras;

> Ao Dr. Marcos Janson e sua fiel escudeira Karina Ducatti, por também, permitirem livre acesso aos seus arquivos de pacientes.

Ao professor Dr. Aiello pela receptividade e contribuição na amostra.

- Ao colega e novo grande amigo Jonas Bianchi pelo auxílio com a amostra do Prof. João Roberto Gonçalves;

Aos colegas Victor Sakima e Letícia Chihara pela ajuda com a amostra do Prof. Dr. Eduardo Sant'Ana; 

Ao amigo e Prof. Dr. Aleysson Olympio Pazza por, gentilmente, ceder seu consultório e computador durante a fase final da pesquisa;

As secretárias: Letícia, Fátima, Leila e Maristela pela paciência e auxílio nas tarefas burocráticas;

$>$ À Faculdade de Odontologia de Bauru, por ser esse centro de referência internacional na formação de grandes acadêmicos, clínicos, pesquisadores e professores.

À CAPES pelo incentivo através da bolsa de Doutorado.

$>\mathrm{E}$ a todos os demais que, direta ou indiretamente, ajudaram a tornar realidade esse grande sonho. 

"A melhor maneira de prever nosso futuro é construí-lo".

Abraham Lincoln 

ABSTRACT 



\section{ABSTRACT}

\section{Long-term stability of surgical-orthodontic open bite treatment: Le Fort I versus 4-piece segmental Le Fort I osteotomy}

Introduction: This vertical malocclusion has the potential of causing functional and esthetic impairment, impacting patients' quality of life negatively. The long-term stability of anterior open bite surgical-orthodontic treatment is an important and controversial issue. A variety of factors such as surgery type, surgery fixation, and the anteroposterior discrepancy is related to and can influence long-term overbite stability. The controversy of stability arises in the inherent difficulty of collecting a homogeneous sample, with considerable sample size and adequate long-term followup, leading the current literature to an inconclusive status. Therefore this thesis aimed to test 2 null hypotheses: 1 - There is no difference in the long-term stability of the surgical-orthodontic correction of anterior open bite when comparing Le Fort I to 4-piece segmental Le Fort I osteotomies. 2- To test the null hypothesis that there is no difference in the long-term stability of the surgical-orthodontic correction of anterior open bite when comparing Class II to Class III patients. Materials and Methods: The sample of the first investigation comprised the lateral cephs of 29 open bite subjects treated with 1-piece Lefort I osteotomy compared to the lateral cephs of 24 open bite subjects treated with 4-piece Lefort I osteotomy; in both groups Class I, II and III subjects were included. The groups were matched regarding age and were compared with t-tests. To test the second null hypothesis, lateral cephs of 21 Class II open bite subjects were compared to lateral cephs of 25 Class III open bite subjects. Overbite changes were compared at 3-time points: T1 (pretreatment), T2 (posttreatment) and T3 (follow-up) by using t-test. Overbite clinical stability percentage at T3 was assessed with the chi-square test. Results: In 1-piece Le Fort I Group $65.52 \%$ of patients presented with clinically significant overbite stability, while in 4-piece Group $83.33 \%$ remained stable in the long-term, however it was not statistically significant. In Class II Group $57.14 \%$ of patients presented with clinically significant overbite stability, while in Class III Group $88 \%$ remained clinically stable, and it was statistically significant. Conclusions: The first null hypothesis regarding 

maxillary segmentation was accepted because there was no significant intergroup difference regarding the percentage of clinically stable patients. The type of fixation seems to influence the long-term stability of open bite surgical-orthodontic correction more than maxillary segmentation. The second null hypothesis was rejected because clinical stability of Class II malocclusion open bite surgical-orthodontic treatment was significantly smaller than in Class III malocclusions.

Keywords: Open bite. Orthognathic surgery. Angle class II. Angle class III 




\section{RESUMO}

\section{Estabilidade em longo prazo do tratamento cirúrgico da mordida aberta anterior por osteotomia tipo Le Fort I com e sem segmentação maxilar}

Introdução: A estabilidade em longo prazo do tratamento orto-cirúrgico da mordida aberta anterior é um assunto relevante e controverso. Esta má oclusão vertical tem o potencial de causar importantes alterações tanto funcionais quanto estéticas, impactando negativamente a qualidade de vida dos pacientes. Vários fatores, como o tipo de cirurgia, a fixação cirúrgica e a discrepância ântero-posterior, estão relacionados e podem influenciar a estabilidade da sobremordida em longo prazo. A controvérsia na estabilidade surge na dificuldade inerente de coletar uma amostra homogênea, com um tamanho de amostra considerável e acompanhamento adequado em longo prazo, levando esse assunto na literatura atual a um status inconclusivo. Portanto, esta tese teve como objetivos testar 2 hipóteses nulas: 1Não há diferença na estabilidade em longo prazo da correção orto-cirúrgica da mordida aberta anterior ao comparar as osteotomias tipo Le Fort I com e sem segmentação maxilar. 2- Não há diferença na estabilidade em longo prazo da correção orto-cirúrgica da mordida aberta anterior ao comparar pacientes com má oclusão de Classe II e Classe III. Materiais e Métodos: A amostra da primeira investigação compreendeu as telerradiografias de 29 indivíduos com mordida aberta tratados por osteotomia Lefort I sem segmentação maxilar, às quais foram comparadas às telerradiografias laterais de 24 indivíduos com mordida aberta tratados por osteotomia Lefort I com segmentação maxilar; pacientes Classe I, II e III foram incluídos. Os grupos foram compatibilizados pela idade e foram comparados com testes $t$ e qui-quadrado. Para testar a segunda hipótese nula, telerradiografias laterais de 21 indivíduos com mordida aberta e má oclusão de Classe II foram comparadas às telerradiografias laterais de 25 indivíduos com mordida aberta e má oclusão de Classe III. As alterações do overbite foram comparadas em três tempos: T1 (pré-tratamento), T2 (pós-tratamento) e T3 (longo-prazo) usando o teste t. 

A taxa de estabilidade clínica em T3 foi avaliada com teste de qui-quadrado. Resultados: No Grupo Le Fort I sem segmentação maxilar 65,52\% dos pacientes apresentaram estabilidade clínica da sobremordida, enquanto que no Grupo Le Fort I com segmentação maxilar, 83,33\% mantiveram-se estáveis em longo prazo, porém essa diferença não se apresentou estatisticamente significante. No Grupo Classe II, $57,14 \%$ dos pacientes permaneceram clinicamente estáveis, enquanto que no Grupo Classe III, a porcentagem clínica de estabilidade foi de $88 \%$, e a diferença apresentou significância estatística. Conclusões: A primeira hipótese nula em relação à segmentação maxilar foi aceita porque não houve diferença estatisticamente significante entre os grupos em relação à porcentagem de pacientes clinicamente estáveis. O tipo de fixação parece influenciar a estabilidade em longo prazo da correção orto-cirúrgica da mordida aberta mais do que a segmentação maxilar. A segunda hipótese nula foi rejeitada porque a estabilidade clínica do tratamento orto-cirúrgico da mordida aberta em pacientes com má oclusão de Classe II foi significativamente menor do que nos pacientes com má oclusão de Classe III.

Palavras-chave: Mordida aberta. Cirurgia ortognática. Classe II de Angle. Classe III de Angle 



\section{TABLE OF CONTENTS}

1 INTRODUCTION

2 PURPOSES

$3 \quad$ ARTICLES

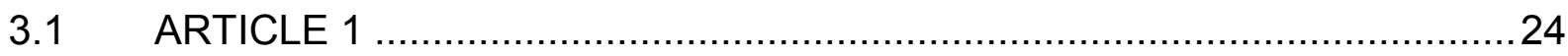

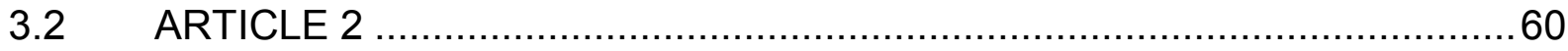

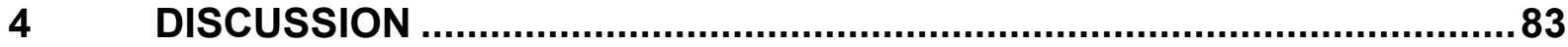

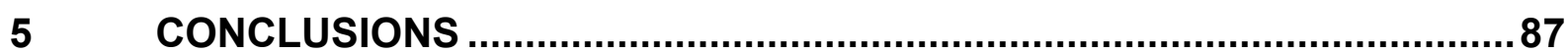

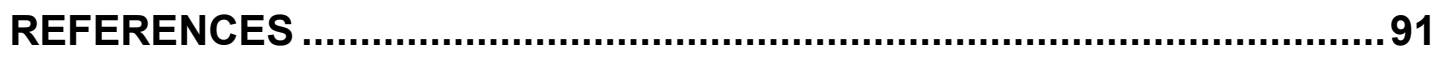

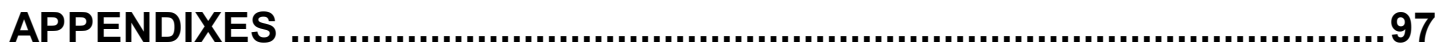

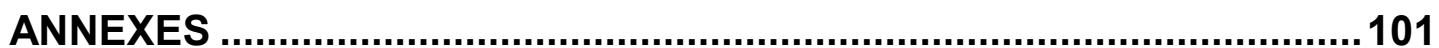



1 INTRODUCTION 



\section{INTRODUCTION}

Orthodontic treatment must be directed to, not only, correct the malocclusion to address patients' esthetic and functional impairments, but also maintaining its results in the long-term.

Treatment of anterior open bite, as well as many other malocclusions, depends on its aetiology, severity and the time that is set, ranging from the simple abandonment of a deleterious habit to an orthognathic surgery involving both the maxilla and mandible (SUBTELNY; SAKUDA, 1964; REYNEKE, 1988; HUANG, 2002; REYNEKE; FERRETTI, 2007; GREENLEE et al., 2011; FONTES et al., 2012).

Even in surgical approaches for open bite treatment, a considerable high relapse rate has been reported in the literature.(HUANG, 2002; MAIA et al., 2010) Besides that, a wide variety of factors are considered to explain the unsuccessful long-term surgical-orthodontic treatment of open bite patients, such as surgery type and anteroposterior discrepancy (HOPPENREIJS et al., 1997; IANNETTI et al., 2007).

Le Fort I impaction approach is a well-established procedure in open bite surgical correction (EPKER; FISH, 1977; BAILEY et al., 1994b; ISMAIL; LEUNG, 2017). This procedure, advantages, and disadvantages have been extensively reviewed in the literature (OBWEGESER, 1970; PROFFIT; WHITE, 2015). Another option is the multi-segmented Le Fort I osteotomy (REYNEKE, 1988; KAHNBERG, 2007; ISMAIL; LEUNG, 2017), allowing the impaction of the maxilla associated with its advance simultaneously correcting transversal, vertical and anteroposterior discrepancies. There is no consensus regarding the stability when these two approaches are compared in the treatment of skeletal open bites (FISCHER; VON KONOW; BRATTSTRÖM, 2000; ARPORNMAEKLONG; HEGGIE; SHAND, 2003; KRETSCHMER et al., 2010; ISMAIL; LEUNG, 2017). In general, segmentation is indicated when there are transversal impairments. Particularly in open bites, some surgeons consider the presence of a 2-step maxillary occlusal plane as a chief-factor to choose the segmental approach. Multi-segmental osteotomies requires more 
surgeon expertise due to the higher risks of damaging dental structure and necrosis at the osteotomy surrounded areas (KAHNBERG; VANNAS-LOFQVIST; ZELLIN, 2005; POSNICK; ADACHIE; CHOI, 2016)

Regarding anteroposterior discrepancy, some studies point out that open bite Class II patients treated surgical-orthodontic ly present a higher overbite relapse rate in the long-term when compared to Class III open bite patients (SWINNEN et al., 2001; MAIA et al., 2010). However, some study design issues, mainly related to sample size limitations and follow-up time, have to lead us to weak evidence on this topic and so motivating these investigations.

There are few consistent and specific studies evaluating the long-term stability of anterior open bite surgical correction (FONTES et al., 2012). Some literature reviews (HUANG, 2002), systematic reviews (SOLANO-HERNANDEZ et al., 2013), and even meta-analyses (GREENLEE et al., 2011; MEDEIROS et al., 2012) were made over the last decade, highlighting the lack of well-designed studies with proper sample sizes and adequate long-term observation period in order to establish a reliable evidence-based status of the stability of the surgical approach when treating open bites. The main problems found in the available studies are related to small sample size, the absence of method error assessment, sample heterogeneity, absence of initial records and short posttreatment time follow-up (SOLANOHERNANDEZ et al., 2013).

Therefore it is of paramount importance to the orthodontist and to the oral surgeon as well, to know if any difference exists regarding the clinical long-term over bite behavior when different surgical approaches are employed in open bite malocclusion surgical-orthodontic treatment, and if the anteroposterior discrepancy also plays a role in the overbite stability. 
2 PURPOSES 



\section{PURPOSES}

The purposes of this thesis are:

1- To test the null hypothesis that there is no difference in the long-term stability of the surgical-orthodontic correction of anterior open bite when comparing Le Fort I to 4-piece segmental Le Fort I osteotomies.

2- To test the null hypothesis that there is no difference in the long-term stability of the surgical-orthodontic correction of anterior open bite when comparing Class II to Class III patients. 

3 ARTICLES 



\section{ARTICLES}

The articles presented in this Thesis were written according to the American Journal of Orthodontics and Dentofacial Orthopedics instructions and guidelines for article submission (Annex E).

- ARTICLE 1 - Long-term stability of surgical-orthodontic open bite treatment: Le Fort I versus 4-piece segmental Le Fort I osteotomy

- ARTICLE 2 - Long-term stability of surgical-orthodontic open bite treatment: Class II versus Class III 


\title{
3.1 ARTICLE 1
}

\section{LONG-TERM STABILITY OF SURGICAL-ORTHODONTIC OPEN BITE TREATMENT: LE FORT I VERSUS 4-PIECE SEGMENTAL LE FORT I OSTEOTOMY}

\begin{abstract}
Introduction: This study aims to test the null hypothesis that there is no difference in the long-term stability of the surgical correction of anterior open bite when comparing Le Fort I to 4-piece segmental Le Fort I osteotomies. Methods: Lateral cephs of 29 open-bite subjects treated with 1-piece Lefort I osteotomy were compared to the lateral cephs of 24 open-bite subjects treated with 4-piece Lefort I osteotomy. Class I, Class II, and Class III subjects were included. The groups were matched regarding age and were compared with t-tests and chi-square tests. Results: In the 1-piece Le Fort I group $65.52 \%$ of patients presented with clinically significant overbite stability, while in the 4-piece group $83.33 \%$ remained stable in the long-term. Conclusions: Despite the higher clinically significant overbite stability percentage on 4-piece Le Fort I group than in 1-piece Le Fort I group, the null hypothesis was accepted because it was not statistically significant. Moreover, the type of fixation seems to influence overbite stability more than the type of maxillary segmentation.
\end{abstract}

\section{INTRODUCTION}

Skeletal anterior open bite often requires surgical correction due to the extreme hyper divergency between the bone bases and its impact on occlusion, phonation, and esthetics, regardless of the severity of this vertical malocclusion. ${ }^{1-4}$ The difficulties of treatment and low stability in cases of anterior open bite, even in surgically treated cases, can lead patients and professionals to frustration. ${ }^{3,5}$

The advancement and development of new surgical techniques can, in theory, bring greater stability in the long-term when treating this challenging malocclusion. ${ }^{6}$ The most severe skeletal discrepancies in anterior open bite malocclusion should be 
corrected surgically, allowing an adequate maxillomandibular position and dentoalveolar interdigitation. ${ }^{7-11}$

A variety of surgical techniques has been used to correct anterior open bite..$^{8,11-13}$ Le Fort I impaction approach often requires the prior execution of surgically assisted rapid maxillary expansion (SARME). Another option is the multi-segmented Le Fort I osteotomy ${ }^{10,14}$, allowing the impaction of the maxilla associated with its advance simultaneously correcting transversal, vertical and anteroposterior discrepancies. In general, segmentation is indicated when there are transversal impairments. Particularly in open bites, the presence of a 2-step maxillary occlusal plane is a chief-factor to choose the segmental approach. There is no consensus in the literature regarding the long-term stability when these two approaches are compared in the treatment of skeletal open bites. ${ }^{12,15}$

There are few consistent and specific studies evaluating the long-term stability of anterior open bite surgical correction ${ }^{8}$. Some literature reviews ${ }^{16}$, systematic reviews $^{17}$, and even meta-analyses ${ }^{5,18}$ were made over the last decade, highlighting the lack of well-designed studies with proper sample sizes and adequate long-term follow-up period to establish a reliable, evidence-based status of the stability of the surgical approach when treating open bites. The main problems found in the available studies are related to small sample size, the absence of method error assessment, sample heterogeneity and absence of initial records. ${ }^{17,19}$

Therefore it is of paramount importance to the orthodontist and to the oral surgeon as well, to know if any difference exists regarding the long-term over bite behavior when different surgical approaches are employed in open bite malocclusion surgical-orthodontic treatment.

The purposes of this study are 1- To test the null hypothesis that there is no difference in the long-term stability of the surgical correction of anterior open bite when comparing Le Fort I (1-piece) to Segmental Le Fort I (4-piece) osteotomies. 2Evaluate skeletal and dentoalveolar changes that can influence open bite relapse. 


\section{MATERIAL AND METHODS}

This study was submitted to and approved by the institutional review board of Bauru Dental School, University of Sao Paulo, Brazil, under the protocol number: CAAE 71636716.5.0000.5417.

Sample size calculation, considering an $80 \%$ test power at a significance level of $5 \%$, with a minimum mean difference to be detected of $1.2 \mathrm{~mm}$ and a mean standard deviation of $1.45 \mathrm{~mm}$, showed that 24 subjects in each of the two groups were the minimum for providing reliable results. The minimum mean difference to be detected and the standard deviation of the variable overbite relapse were based on a previous study. ${ }^{13}$

Inclusion criteria comprised: initial anterior open-bite malocclusion of at least $1 \mathrm{~mm}$, treated orthodontically and surgically with 1-piece Le Fort I or 4-piece Le Fort I osteotomies, no previous orthodontic treatment; positive overbite after treatment; comparable retention protocols; facial trauma absence; buccofacial pathology absence, genetic syndromes/ congenital deformities absence;

Lateral cephs were taken at three stages: pretreatment (T1), posttreatment (T2 - immediately after debonding), and long-term follow-up (T3 - at least two years after debonding). The presurgical orthodontic treatment comprised leveling and alignment with fixed preadjusted appliances. Intermaxillary fixation with elastics was indicated in the postsurgical phases during seven weeks. Seven different professional teams treated patients. Regarding retention protocol, a Hawley retainer was recommended during the first 12 posttreatment months, and a canine-to-canine retainer to control the mandibular incisor alignment for a mean period of 2 years. Open bite relapse was considered as a negative overbite showed at T3. Only six patients presented with TMD symptoms and three required TMJ disc repositioning. ${ }^{20,21}$

The sample was divided into two groups:

Group 1: 1-piece Le Fort I comprised 29 patients (11 male, 18 female) with a mean age of 23.22 years (ranging from 16.83 years to 37.91 years). Regarding anteroposterior discrepancy, this group presented 4 Class I, 12 Class II and 13 Class III patients. In only 6 patients orthognathic surgery was restricted to the maxilla, while the remaining 23 patients were treated by combining 1-piece Le Fort I to BSSO 
(Bilateral Sagittal Split Osteotomy). Surgical fixation with intraosseous wires (WF) was performed in 17 patients (58.62\%), and $12(41.38 \%)$ received rigid internal fixation. Patients in this group were treated by the same professional team, including only two surgeons. The posttreatment follow-up had a mean time of 7.74 years (ranging from 2 years to 18.83 years). Due to the presence of two different types of bone fixation (Wires and RIF) in Group 1 and the possible influence that it could have in the long-term stability, ${ }^{22}$ this group was divided into two subgroups, described as follows:

Subgroup 1 (WF): comprising 17 patients (5 male, 12 female) with a mean age of 22.50 years (ranging from 16.83 years to 37.91 years). Regarding anteroposterior discrepancy, this group presented 3 Class I, 8 Class II and 6 Class III patients. In 4 patients, orthognathic surgery was restricted to the maxilla, while the remaining 13 patients were treated by combining 1-piece Le Fort I to BSSO (Bilateral Sagittal Split Osteotomy). Patients in this group were treated by the same professional team, including only two surgeons. The posttreatment follow-up interval had a mean time of 10.65 years (ranging from 4 years to 18.83 years).

Subgroup 2 (RIF): comprising 12 patients (6 male, 6 female) with a mean age of 24.25 years (ranging from 14.83 years to 37.91 years). Regarding anteroposterior discrepancy, this group presented 1 Class I, 4 Class II and 7 Class III patients. In 2 patients, orthognathic surgery was restricted to the maxilla, while the remaining 10 patients were treated by combining 1-piece Le Fort I to BSSO (Bilateral Sagittal Split Osteotomy). Patients in this group were treated by the same professional team, including only two surgeons. The posttreatment follow-up interval had a mean time of 3.61 years (ranging from 2 years to 6.84 years).

Group 2: 4-piece Le fort I group comprised 24 patients (16 male, eight female) with a mean age of 26.30 years (ranging from 14.83 years to 48.66 years). Regarding anteroposterior discrepancy, this group presented 3 Class I, 9 Class II and 12 Class III patients. In only 2 patients orthognathic surgery was restricted to the maxilla, while the remaining 22 patients were treated by combining 4-piece Le Fort I to BSSO (Bilateral Sagittal Split Osteotomy). Surgical fixation with rigid internal fixation (RIF) was performed in all patients in this group. Patients in this group were treated by 5 different professional teams, including 5 surgeons, as described: surgeon \# 1(3 patients), surgeon \# 2 (7 patients), surgeon \# 3 (6 patients), surgeon \# 
4 (4 patients) and surgeon \# 5 (4 patients). Retention included a maxillary Hawley plate during the first posttreatment year and a bonded mandibular canine-to-canine retainer for a mean period of 2 years. The posttreatment follow-up had a mean time of 3.71 years (ranging from 2 years to 16.75 years).

\section{Methods}

Lateral cephs of each patient were obtained at T1, T2, and T3, and due to a long time passed between patient recalls, several x-ray machines and magnification factors were present, varying from $6 \%$ to $11.6 \%$. Cephalometric tracings and magnification adjustment were performed by using Dolphin $®$ Imaging 11.0 (Dolphin Imaging and Management Solutions, Chatsworth, Calif., EUA).

In total, 23 measurements (11 angulars, 12 linear), divided between the maxillary component, mandibular component, maxillomandibular relationship, growth pattern, maxillary dentoalveolar component, mandibular dentoalveolar component and dental relationships were performed (Table I) to evaluate skeletal and dentoalveolar features.

\section{Error study}

Error study assessment comprised 32 of the 159 Lateral cephs which were randomly selected and measured again, by the same operator (VLBS), after a minimum 30-day interval. Random errors were calculated according to Dahlberg's formula $\left(\mathrm{Se} 2=\sum \mathrm{d} 2 / 2 \mathrm{n}\right),{ }^{23}$, and the systematic errors with dependent t-tests, at $P<0.05 .^{24-26}$

\section{Statistical analyses}

To assess normal distribution of data in the sample, Kolmogorov-Smirnov tests were performed. All variables showed normal distributions in the two groups.

Chi-square test was used to compare the groups regarding sex distribution, and the percentages of patients with clinically significant stability/relapse in each group and subgroups. 
T-tests were used to evaluate the intergroup comparability of age, and all variables at T1, T2-T1, T2, T3-T2, and T3. Posttreatment time of those subjects that presented overbite clinical stability was compared to those who relapsed in Group 1 (1-piece) by using t-tests.

Results were considered significant at $P<0.05$. All statistical analyses were performed with Statistica software (Statistica for Windows 10.0; StatSoft, Tulsa, Okla.).

\section{RESULTS}

Three variables presented with significant systematic error (Mx1.SN; Mx1-NA; Interincisal angle), requiring caution when taking these measurements into account. Random errors varied within acceptable limits from $0.20 \mathrm{~mm}$ (overbite) to $3.28 \mathrm{~mm}$ $(\mathrm{CoA}){ }^{25}$ Sex distribution presented a statistically significant difference between groups at T1 (Table II).

\section{Group 1 (1-piece) x Group 2 (4-piece):}

Group 1 (1-piece) presented longer treatment and posttreatment times than Group 2 (4-piece) at T1 (Table III) significantly.

Treatment changes results showed, in both groups, that A-Nperp (mm), SNB angle and Inter-incisal angle increased with treatment, but to a lesser extent in Group 1(1-piece) than in Group 2 (4-piece) (Table IV). FMA, SNGoGn, and Md1-Apo (mm) decreased with treatment, in both groups, but to a lesser extent in Group 1(1-piece) than in Group 2 (4-piece) (Table IV). Md1-MP (mm) and Md6-MP (mm) measurements increased in Group 1(1-piece) while decreased in Group 2 (4-piece) (Table IV).

At the end of treatment, IMPA, ANB, and overjet presented higher values in Group 1(1-piece) than in Group 2 (4-piece) at T2 (Table V). Inter-incisal angle presented smaller value in Group 1(1-piece) than in Group 2 (4-piece) at T2 (Table $\mathrm{V})$.

Posttreatment changes were similar between groups.

After follow-up, IMPA, ANB, and Md6-MP ( $\mathrm{mm}$ ) presented greater values in Group 1(1-piece) than in Group 2 (4-piece) at T3 (Table VII). Mx1-NA, and interincisal angle presented positive smaller values in Group 1(1-piece) than in Group 2 (4-piece) at T3 (Table VII). 


\section{Overbite behavior}

Overbite remained positive at T3. Group 1 (1-piece) presented a smaller positive and statistically significant mean of $0.14 \mathrm{~mm}$ than Group 2 (4-piece) which was $1 \mathrm{~mm}$ at T3. Although the difference in overbite mean, stability percentages were not statistically significant different: Group 1 (1-piece) $65.52 \%$ and Group 2 (4-piece) $83.33 \%$.

\section{Subgroup 1 (WF) x Group 2 (4-piece)}

Regarding treatment time and posttreatment time duration, Subgroup 1(WF) presented a significantly longer period than Group 2 (4-piece) at T1 (Table VIII).

Treatment changes results showed, in both groups, that SNA, A-Nperp $(\mathrm{mm})$, Inter-incisal angle increased with treatment, but to a lesser extent in Subgroup 1(WF) than in Group 2 (4-piece) (Table IX). FMA and SNGoGn decreased with treatment, but to a lesser extent in Subgroup 1(WF) than in Group 2 (4-piece) (Table IX). Md1MP $(\mathrm{mm})$ and Md6-MP $(\mathrm{mm})$ measurements increased with treatment in Subgroup 1(WF) while decreased in Group 2 (4-piece) (Table IX).

At the end of treatment, IMPA, ANB, FMA, and Md1-Apo $(\mathrm{mm})$ presented higher values in Subgroup 1(WF) than in Group 2 (4-piece) at T2 (Table X). Interincisal angle presented smaller value in Subgroup 1(WF) than in Group 2 (4-piece) at T2 (Table X).

Posttreatment changes were similar between groups.

After follow-up, ANB and FMA presented greater in Subgroup 1(WF) than in Group 2 (4-piece) at T3 (Table XII). Pog-NB (mm), Mx1-NA (mm) and inter-incisal angle presented smaller values in Subgroup 1(WF) than in Group 2 (4-piece) at T3 (Table XII).

\section{Overbite behavior}

Overbite returned to a negative mean only in Subgroup 1 (WF) at T3. Subgroup 1 (WF) presented a negative and statistically significant mean of $-0.18 \mathrm{~mm}$ than Group 2 (4-piece) which was $1 \mathrm{~mm}$ at T3. Moreover, clinical stability percentage in Subgroup 1 (WF) (52.94\%) was also statistically significant smaller than Group 2 (4-piece) (83.33\%) (Table XII). 


\section{Subgroup 2 (RIF) x Group 2 (4-piece):}

Regarding treatment time duration, Subgroup 2 (RIF) presented a significantly longer period than Group 2 (4-piece) at T1 (Table XIII).

Treatment changes results showed, in both groups, that FMA, MP-SN, and Md1-APo decreased with treatment, but to a lesser extent in Subgroup 2 (RIF) than in Group 2 (4-piece) (Table XIV). SN-PP, SN-OP, Md1-MP (mm) and Md6-MP (mm) measurements increased with treatment in Subgroup 2 (RIF) while decreased in Group 2 (4-piece) (Table XIV).

At the end of treatment, Mx6-PTV $(\mathrm{mm})$ presented higher value in Subgroup 2 (RIF) than in Group 2 (4-piece) at T2 (Table XV). Inter-incisal angle presented smaller value in Subgroup 2 (RIF) than in Group 2 (4-piece) at T2 (Table XV).

Posttreatment changes were similar between groups.

After follow-up, IMPA presented higher value in Subgroup 2 (RIF) than in Group 2 (4-piece) at T3 (Table XVII). Inter-incisal angle presented smaller value in Subgroup 2 (RIF) than in Group 2 (4-piece) at T3 (Table XVII).

\section{Overbite behavior}

Overbite remained positive at T3 in Subgroup 2 (RIF), with a mean of $0.60 \mathrm{~mm}$ and in Group 2 (4-piece) it was $1 \mathrm{~mm}$. Besides, clinical stability percentages were the same in both groups (83.33\%) (Table XVII).

\section{Posttreatment time comparison in Group 1 (1-piece)}

Posttreatment time comparison of those subjects who remained clinically stable to those who relapsed showed no statistically significant difference (Table $\mathrm{XVIII).}$

\section{DISCUSSION}

\section{Sample}

The groups were not comparable regarding sex distribution. However, this is not a problem since overbite behavior shows no difference between sexes. ${ }^{27,28}$ Our sample comprises a considerable number of specific open bite subjects distributed 
among groups and subgroups, while other studies had not that degree of specification. ${ }^{29,30}$ Such characteristic, allows the isolation of long-term stability potential influencing factors, such as maxillary segmentation, type of fixation, anteroposterior discrepancy, and operator bias with a proper number of individuals. Besides, at T1, mostly initial records were included, in spite of pre-surgical ones, ${ }^{12,31}$ this is important because the initial open bite severity must be measured, avoiding the possible influence of pre-surgical orthodontics (posterior tooth intrusion/anterior tooth extrusion). According to most authors, ${ }^{4,32-34}$ orthodontic preparation can influence post-surgical stability, while others disagree. ${ }^{35,36}$. Due to the variability in the duration of post-surgical orthodontic treatment and the influence of the use of intermaxillary anterior elastics exerts on overbite maintenance, a mean long-term follow-up of 5.92 years after appliance removal and not immediately after surgery, as we find in some studies, ${ }^{12,31}$ is also a strong feature present in this sample.

Operator bias (number and expertise of professionals) is regarded as a factor which could influence treatment outcome when surgical procedures are assessed, however just a few studies really evaluated this condition. According to this rationale, in our study, Group 1 (1-piece), which presented more homogeneity on this issue (only two oral surgeons), should demonstrate higher rates of stability than in Group 2 (4-piece), comprising five different oral surgeons. Thus our results contradict this tendency. The expertise of the operators is more important than the number of professionals involved in the research. ${ }^{37}$ Despite a considerable high number of different professionals have participated in the sample, only experienced orthodontists and oral surgeons with academic background conducted all treatments. Again, is imperative to highlight the difficulty of retrospectively select a sample with such specific characteristics. Moreover, this diversity regarding operators can show us a more realistic Prisma on the long-term stability of surgical-orthodontic open bite treatment, since data was collected in private practices across the country.

Another characteristic of our sample that could have influenced the results is that the patients in Group 1 (1-piece) presented a statistically significant greater mean in the posttreatment time of 7.74 years, than Group 2 (4-piece) which was 3.71 years (Table III). This fact, give us the chance to speculate that, based on the literature, ${ }^{9,38}$ the patients in Group 2 (4-piece) have a higher chance of relapse over time than those in Group 1 (1-piece) because the posttreatment time was less than 
five years. Moreover, if we take a closer look at the posttreatment time of those subjects who relapsed in Group 1 (1-piece), we found an increase in the mean to 9.01 years when compared to the whole Group 1 (1-piece) mean which was 7.74 years. Conversely, looking at those in Group 1 (1-piece) who remained stable we found a mean of 7.07 years, which is closer to the whole Group 1 (1-piece) mean which was 7.74 years (Table XVIII). However, there was no statistically significant difference when the posttreatment times of those patients who relapsed were compared to those who remained stable in Group 1 (1-piece) (Table XVIII). In Group 2 (4-piece), contrary to the results of Group 1 (1-piece), the relapsed subjects had a smaller posttreatment time of 2.64 years than the whole Group 2 (4-piece) mean which was 3.71 years. Whereas the stable ones presented with a greater mean value of 4.87 years, no statistically significant difference was detected (Table XVIII). So, the available data does not allow us to clarify this speculation. Hence, specific studies about this issue should be performed to assess this tendency.

Treatment changes regarding growth pattern measurements (FMA and SN.GoGn) and SNB were smaller in Group 1(1-piece) than in Group 2 (4-piece) (Table IV), hence the relapse tendency should be lesser in Group 1 (1-piece) than in Group 2 (4-piece), however, the posttreatment phase was similar between groups (Table VI).

\section{Overbite stability}

Due to the variability of results found in previous studies, ${ }^{9,29,31,35,39,40}$ the main objective of this study was to compare the long-term clinical stability/relapse of surgical-orthodontic treatment in a specific sample of open bite patients between two different surgical approaches. Regarding the skeletal stability, some authors concluded that no difference was found on the vertical plane between 1-piece Le Fort I and 3-piece Le Fort I osteotomies. ${ }^{7,35,39}$ While others, considering the final overbite result regardless of what led to the overbite stability/instability, found that 1-piece was more stable ${ }^{31,41}$ and some others considered 1-piece less stable than segmental ones such as 3-piece. ${ }^{12,19,29}$

Our results, although demonstrated a statistically significant greater overbite value of $1 \mathrm{~mm}$ at T3 (Table VII) in Group 2 (4-piece) than in Group 1 (1-piece) which was $0.06 \mathrm{~mm}$, showed that overbite means in both groups remained positive after 
follow-up. When the percentages of stable subjects are observed, despite the higher stability percentage in Group $2(83.33 \%)$ than in Group 1(65.52\%), results were not statistically significant (Table VII). In Group 1 (1-piece), 19 out of 29 patients remained clinically stable (overbite greater than $0 \mathrm{~mm}$ ) and 10 presented with a clinically overbite relapse (overbite measurement smaller than $0 \mathrm{~mm}$ ), whereas 20 out of 24 patients remained clinically stable (overbite greater than $0 \mathrm{~mm}$ ) after the longterm observation period and 4 presented with a clinical overbite relapse (overbite measurement smaller than $0 \mathrm{~mm}$ ) in Group 2 (4-piece) (Table VII).

Because in Group 2 (4-piece) all patients received R.I.F whereas in Group 1 (1-piece) patients were treated with different types of fixation (intra-osseous wires/rigid internal fixation) and there is strong evidence that it can influence longterm stability of surgical procedures, ${ }^{7}$ Group 1 (1-piece) was divided into two subgroups (Subgroup 1 (WF) and Subgroup 2 (RIF)). The Subgroup 1 (WF) to Group 2 (4-piece) comparison produced quite different results. Nine out of 17 patients remained clinically stable (overbite greater than $0 \mathrm{~mm}$ ) after the long-term observation period and 8 presented with a clinical overbite relapse (overbite measurement smaller than $0 \mathrm{~mm}$ ). Overbite mean in Subgroup 1 (WF) became negative after followup $(-0.14 \mathrm{~mm})$, increasing the existing statistically significant difference in the overbite at T3 (Table XII). Moreover, regarding the percentages of stable subjects in Subgroup 1 (WF), it dropped to $52.94 \%$ turning the difference statistically significant (Table XII).

The Subgroup 2 (RIF) to Group 2 (4-piece) comparison indicated that the type of fixation exerts an influence on the long-term stability of open bite surgicalorthodontic correction corroborating other investigations. ${ }^{2,4,7}$ Both groups presented positive and similar overbite means (Subgroup $2(0.35 \mathrm{~mm})$ and Group $2(1 \mathrm{~mm}))$ at T3 (Table XVII). The percentage of clinically stable overbite was the same in both groups $(83.33 \%)$ hence results were not statistically significant (Table XVII). Ten out of 12 patients remained clinically stable (overbite greater than $0 \mathrm{~mm}$ ) after the longterm observation period and only 2 presented with a clinical overbite relapse (overbite measurement smaller than $0 \mathrm{~mm}$ ) (Table XVII).

Posttreatment changes were similar among all groups and subgroups, however, in the Subgroup 1 (WF) to Group 2 (4-piece) comparison, a stronger tendency in overbite decrease in Group $1(-0.92 \mathrm{~mm})$ was observed compared to 
Group $2(-0.10 \mathrm{~mm})$ with a $\mathrm{p}=0.095$ (Table XI). Even though, Subgroup 2 (RIF) has only 12 patients whereas Group 2 (4-piece) has 24, they presented very similar results in all treatment phases, mainly in the posttreatment follow-up (Tables XII to $\mathrm{XVII}$ ), revealing the strong influence of the type of fixation in the overall results of Group 1 (1-piece). 


\section{CONCLUSIONS}

The null hypothesis was accepted because:

- There was no significant intergroup difference regarding the percentage of clinically stable patients;

- The type of fixation seems to influence the long-term stability of open bite surgical-orthodontic correction more than maxillary segmentation. 


\section{REFERENCES}

1-Epker BN. Modifications in the saggital osteotomy of the mandible. J Oral Surg 1977;35:157-159.

2-Bailey LTJ, Cevidanes LHS, Proffit WR. Stability and predictability of orthognathic surgery. Am J Orthod Dentofacial Orthop 2004;126:273-277.

3-Maia FA, Janson G, Barros SE, Maia NG, Chiqueto K, Nakamura AY. Longterm stability of surgical-orthodontic open-bite correction. Am J Orthod Dentofacial Orthop 2010;138:254 e251-254 e210; discussion 254-256.

4-Proffit WR, White J. Combined surgical-orthodontic treatment: How did it evolve and what are the best practices now? Am J Orthod Dentofacial Orthop 2015;147:205-215.

5-Greenlee GM, Huang GJ, Chen SS, Chen J, Koepsell T, Hujoel P. Stability of treatment for anterior open-bite malocclusion: a meta-analysis. Am J Orthod Dentofacial Orthop 2011;139:154-169.

6-Silva I, Suska F, Cardemil C, Rasmusson L. Stability after maxillary segmentation for correction of anterior open bite: a cohort study of 33 cases. J Craniomaxillofac Surg 2013;41:e154-158.

7-Hoppenreijs TJM, Freihofer HPM, Stoelinga PJW, Tuinzig DB, van't Hof MA, Nottet SJAM. Skeletal and dentoalveolar stability of Le Fort I intrusion osteotomies and bimaxillary osteotomies in anterior open bite deformities. A retrospective threecentre study. Int J Oral Maxillofac Surg 1997;26:161-175.

8-Fontes AM, Joondeph DR, Bloomquist DS, Greenlee GM, Wallen TR, Huang GJ. Long-term stability of anterior open-bite closure with bilateral sagittal split osteotomy. Am J Orthod Dentofacial Orthop 2012;142:792-800. 
9-Proffit WR, Turvey TA, Phillips C. The hierarchy of stability and predictability in orthognathic surgery with rigid fixation: an update and extension. Head Face Med $2007 ; 3: 21$.

10-Reyneke JP. Vertical variation in skeletal open bite: a classification for surgical planning. J Dent Ass S Africa 1988;43:465-472.

11-Reyneke JP, Ferretti C. Anterior open bite correction by Le Fort I or bilateral sagittal split osteotomy. Oral Maxillofacial Surg Clin N Am 2007;19:321-338.

12-Arpornmaeklong P, Heggie AA, Shand JM. A comparison of the stability of single-piece and segmental Le Fort I maxillary advancements. J Craniofac Surg 2003;14:3-9.

13-Espeland L, Dowling PA, Mobarak KA, Stenvik A. Three-year stability of open-bite correction by 1-piece maxillary osteotomy. Am J Orthod Dentofacial Orthop 2008;134:60-66.

14-Kahnberg KE. Transverse expansion of the maxilla using a multisegmentation technique. Scand J Plast Reconstr Surg Hand Surg 2007;41:103108.

15-Fischer K, von Konow L, Brattström V. Open-bite: stability after bimaxillary surgery-2-year treatment outcomes in 58 patients. Eur J Orthod 2000;22:711-718.

16-Huang GJ. Long-term stability of anterior open-bitetherapy: A review Semin Orthod: Elsevier; 2002: p. 162-172.

17-Solano-Hernandez B, Antonarakis GS, Scolozzi P, Kiliaridis S. Combined orthodontic and orthognathic surgical treatment for the correction of skeletal anterior open-bite malocclusion: a systematic review on vertical stability. J Oral Maxillofac Surg 2013;71:98-109. 
18-Medeiros RB, de Araújo LFC, Mucha JN, Motta AT. Stability of open-bite treatment in adult patients: A systematic review. World J Orthod 2012;1:e97-e101.

19-Ismail I, Leung Y. Anterior open bite correction by Le Fort I osteotomies with or without anterior segmentation: which is more stable? Int $\mathrm{J}$ Oral Maxillofac Surg 2017;46:159.

20-Goncalves JR, Cassano DS, Wolford LM, Santos-Pinto A, Marquez IM. Postsurgical stability of counterclockwise maxillomandibular advancement surgery: affect of articular disc repositioning. J Oral Maxillofac Surg 2008;66:724-738.

21-Bianchi J, Pinto ADS, Ignacio J, Obelenis Ryan DP, Goncalves JR. Effect of temporomandibular joint articular disc repositioning on anterior open-bite malocclusion: An orthodontic-surgical approach. Am J Orthod Dentofacial Orthop 2017;152:848-858.

22-Forssell K, Turvey TA, Phillips C, Proffit WR. Superior repositioning of the maxilla combined with mandibular advancement: mandibular RIF improves stability. Am J Orthod Dentofacial Orthop 1992;102:342-350.

23-Dahlberg G. Statistical methods for medical and biological students. New York: Interscience; 1940.

24-Houston W. The analysis of errors in orthodontic measurements. Am J Orthod 1983;83.

25-Baumrind S, Frantz RC. The reliability of head film measurements: 1. Landmark identification. Am J Orthod 1971;60.

26-Richardson A. A classification of open bites. Eur J Orthod 1981;3.

27-Bishara SE, Jakobsen JR. Changes in overbite and face height from 5 to 45 years of age in normal subjects. Angle Orthod 1998;68:209-216. 
28-Bergersen EO. A Longitudinal Study of Anterior Vertical Overbite from Eight to Twenty Years of Age. Angle Orthod 1988;58:237-256.

29-Turvey TA, Philips C, Zaytoun HS, Proffit WR. Simultaneous superior repositioning of the maxilla and mandibular advancement. Am J Orthod 1988;94:372383.

30-McCance A, Moss J, James D. Stability of surgical correction of patients with skeletal III and skeletal II anterior open bite, with increased maxillary mandibular planes angle. Eur J Orthod 1992;14:198-206.

31-Kretschmer WB, Baciut G, Baciut M, Zoder W, Wangerin K. Stability of Le Fort I osteotomy in bimaxillary osteotomies: single-piece versus 3-piece maxilla. J Oral Maxillofac Surg 2010;68:372-380.

32-Ellis E, 3rd. The nature of vertical maxillary deformities: implications for surgical intervention. J Oral Maxillofac Surg 1985;43:756-762.

33-Lines PA, Steinhauser EW. Diagnosis and treatment planning in surgical orthodontic therapy. Am J Orthod 1974;66:378-397.

34-Nemeth RB, Isaacson RJ. Vertical anterior relapse. Am J Orthod 1974;65:565-585.

35-Perez MMC, Sameshima GT, Sinclair PM. The long-term stability of LeFort I maxillary downgrafts with rigid fixation to correct vertical maxillary deficiency. Am J Orthod Dentofacial Orthop 1997;112:104-108.

36-Lo FM, Shapiro PA. Effect of presurgical incisor extrusion on stability of anterior open bite malocclusion treated with orthognathic surgery. Int J Adult Orthodon Orthognath Surg 1998;13:23-34. 
37-Porter GA, Soskolne CL, Yakimets WW, Newman SC. Surgeon-related factors and outcome in rectal cancer. Annals of surgery 1998;227:157.

38-Maia FA, Janson G, Barros SE, Maia NG, Chiqueto K, Nakamura AY. Editor's Comment and Q\&A: Long-term stability of surgical-orthodontic open-bite correction. Am J Orthod Dentofac Orthop 2010;138:254-256.

39-Bailey L, Phillips C, Proffit W, Turvey T. Stability following superior repositioning of the maxilla by Le Fort I osteotomy: five-year follow-up. Int $\mathrm{J}$ Adult Orthod Orthognath Surg 1994;9:163-173.

40-Proffit WR, Bailey LJ, Phillips C, Turvey TA. Long-term stability of surgical open-bite correction by Le Fort I osteotomy. Angle Orthod 2000;70:112-117.

41-Fischer K, von Konow L, Brattstrom V. Open bite: stability after bimaxillary surgery--2-year treatment outcomes in 58 patients. Eur J Orthod 2000;22:711-718. 
Table I. Cephalometric variables description.

\begin{tabular}{|c|}
\hline Maxillary component \\
\hline 1. SNA angle $\left(^{\circ}\right)$ SN to NA. \\
\hline 2. Co-A (mm) Condylion to A-point. \\
\hline 3. A-Nperp (mm) A-point to nasion-perpendicular. \\
\hline Mandibular component \\
\hline 4. SNB angle $\left({ }^{\circ}\right)$ SN to $N B$. \\
\hline 5. IMPA $\left({ }^{\circ}\right)$ Incisor mandibular plane angle \\
\hline 6. Pog-NB (mm) Pogonion to NB \\
\hline 7. $\mathrm{GoGn} . \mathrm{OP}\left({ }^{\circ}\right)$ Mandibular plane to occlusal plane \\
\hline Maxillomandibular relationship \\
\hline 8. ANB angle $\left(^{\circ}\right)$ NA to NB. \\
\hline $\begin{array}{l}\text { 9. Wits appraisal }(\mathrm{mm}) \text { Distance between perpendicular projections of Points } A \text { and } B \\
\text { on the functional occlusal plane (plane drawn through points of occlusal contact } \\
\text { between the first permanent molars and the first and second premolars). }\end{array}$ \\
\hline Growth pattern \\
\hline 10. FMA $\left(^{\circ}\right)$ Frankfort mandibular plane angle. \\
\hline 11. SN.GoGn $\left(^{\circ}\right)$ SN to GoGn angle. \\
\hline 12. SN.PP $\left(^{\circ}\right)$ SN to palatal plane angle. \\
\hline 13. SN.OP $\left(^{\circ}\right)$ SN to functional occlusal plane angle. \\
\hline Maxillary dentoalveolar component \\
\hline 14. Mx1.SN $\left(^{\circ}\right)$ Angle between maxillary incisor long axis and SN \\
\hline 15. Mx1-NA (mm) Distance from incisal edge of maxillary incisor to NA \\
\hline 16. Mx1Tip-VRP $(\mathrm{mm})$ Distance from incisal edge of maxillary incisor to SN \\
\hline 17. Mx6-PTV $(\mathrm{mm})$ Distance from mesial cusp of maxillary first molar to PTV \\
\hline Mandibular dentoalveolar component \\
\hline $\begin{array}{l}\text { 18. Md1-MP }(\mathrm{mm}) \text { Perpendicular distance between incisal edge of mandibular incisor and } \\
\text { mandibular plane. }\end{array}$ \\
\hline $\begin{array}{l}\text { 19. Md6-MP }(\mathrm{mm}) \text { Perpendicular distance between mesial cusp of mandibular first molar } \\
\text { and mandibular plane. }\end{array}$ \\
\hline $\begin{array}{l}\text { 20. Md1-Pogperp (mm) Perpendicular distance between incisal edge of mandibular } \\
\text { central incisor and Pogperp (line perpendicular to mandibular plane, passing through } \\
\text { pogonion). }\end{array}$ \\
\hline Dental relationships \\
\hline $\begin{array}{l}\text { 21. Overbite }(\mathrm{mm}) \text { Distance between incisal edges of maxillary and mandibular central } \\
\text { incisors, perpendicular to functional occlusal plane. }\end{array}$ \\
\hline $\begin{array}{l}\text { 22. Overjet (mm) Distance between incisal edges of maxillary and mandibular central } \\
\text { incisors, parallel to functional occlusal plane. }\end{array}$ \\
\hline $\begin{array}{l}\text { 23. Interincisal angle }\left({ }^{\circ}\right) \text { Angle between the intersection of maxillary and mandibular } \\
\text { incisors long axis }\end{array}$ \\
\hline
\end{tabular}


Table II. Intergroup comparability regarding sex (chi-square).

\begin{tabular}{|c|c|c|c|}
\hline Group & Male & Female & Total \\
\hline G1(1-piece) & 11 & 18 & 29 \\
\hline G2(4-piece) & 16 & 8 & 24 \\
\hline Total & 27 & 26 & 53 \\
\hline \multicolumn{2}{|c|}{$\mathbf{P = 0 . 0 0 0 ^ { * }}$} \\
\hline
\end{tabular}

${ }^{*}$ Statistically significant at $P<0.05$ 
Table III. Group 1 (1-Piece) to Group 2 (4-piece) comparability regarding age, treatment time, posttreatment time and cephalometric variables at T1 (t test).

\begin{tabular}{|c|c|c|c|c|c|}
\hline \multirow[t]{2}{*}{ Variables } & \multicolumn{2}{|c|}{$\begin{array}{c}\text { Group } 1 \text { (1-Piece) } \\
(\mathrm{N}=29)\end{array}$} & \multicolumn{2}{|c|}{$\begin{array}{c}\text { Group } 2 \text { (4-Piece) } \\
(\mathrm{N}=24)\end{array}$} & \multirow[t]{2}{*}{$\mathbf{P}$} \\
\hline & Mean & s.d & Mean & s.d & \\
\hline Age $(y)$ & 23.22 & 5.79 & 26.30 & 9.92 & 0.164 \\
\hline Treatment time $(\mathrm{y})$ & 3.54 & 2.56 & 1.27 & 1.23 & $0.000^{*}$ \\
\hline Posttreatment time $(\mathrm{y})$ & 7.74 & 4.55 & 3.71 & 3.20 & $0.000^{*}$ \\
\hline \multicolumn{6}{|l|}{ Sex } \\
\hline \multicolumn{6}{|l|}{ Maxillary component } \\
\hline SNA $\left(^{\circ}\right)$ & 82.64 & 4.67 & 78.17 & 5.63 & $0.002^{*}$ \\
\hline CoA (mm) & 80.06 & 3.86 & 77.80 & 8.61 & 0.210 \\
\hline A-Nperp (mm) & 1.92 & 4.42 & -0.58 & 4.06 & $0.038^{*}$ \\
\hline \multicolumn{6}{|l|}{ Mandibular component } \\
\hline SNB $\left(^{\circ}\right)$ & 78.93 & 5.49 & 76.52 & 7.95 & 0.198 \\
\hline $\operatorname{IMPA}\left({ }^{\circ}\right)$ & 87.89 & 9.55 & 84.09 & 7.54 & 0.119 \\
\hline Pog-NB (mm) & 1.37 & 1.73 & 1.76 & 1.89 & 0.438 \\
\hline GoGn.OP( $\left.{ }^{\circ}\right)$ & 23.53 & 4.93 & 25.70 & 6.73 & 0.182 \\
\hline \multicolumn{6}{|c|}{ Maxillomandibular relationship } \\
\hline ANB $\left(^{\circ}\right)$ & 3.71 & 5.93 & 1.70 & 5.33 & 0.205 \\
\hline Wits appraisal (mm) & -1.59 & 8.05 & -2.36 & 6.87 & 0.711 \\
\hline \multicolumn{6}{|l|}{ Growth pattern } \\
\hline $\mathrm{FMA}\left({ }^{\circ}\right)$ & 31.35 & 4.90 & 32.90 & 10.07 & 0.468 \\
\hline SN.GoGn $\left({ }^{\circ}\right)$ & 40.82 & 5.82 & 44.22 & 11.48 & 0.169 \\
\hline SN.PP( $\left(^{\circ}\right)$ & -0.50 & 3.48 & 0.29 & 4.36 & 0.459 \\
\hline SN.OP $\left(^{\circ}\right)$ & 17.28 & 4.42 & 18.52 & 7.67 & 0.464 \\
\hline \multicolumn{6}{|c|}{ Maxillary dentoalveolar component } \\
\hline $\mathrm{M} \times 1 . \mathrm{SN}\left({ }^{\circ}\right)$ & 107.94 & 7.59 & 106.66 & 9.21 & 0.583 \\
\hline Mx1-NA (mm) & 4.83 & 3.36 & 6.65 & 3.16 & $0.048^{*}$ \\
\hline Mx1Tip-VRP (mm) & 69.25 & 6.97 & 65.67 & 10.78 & 0.150 \\
\hline Mx6-PTV (mm) & 21.50 & 5.28 & 21.14 & 5.82 & 0.813 \\
\hline \multicolumn{6}{|c|}{ Mandibular dentoalveolar component } \\
\hline $\mathrm{Md} 1-\mathrm{MP}(\mathrm{mm})$ & 39.76 & 3.97 & 40.35 & 4.64 & 0.623 \\
\hline $\mathrm{Md6}-\mathrm{MP}(\mathrm{mm})$ & 31.25 & 3.54 & 31.57 & 4.03 & 0.760 \\
\hline Md1-Apo (mm) & 4.30 & 2.79 & 4.81 & 2.41 & 0.485 \\
\hline \multicolumn{6}{|l|}{ Dental relationships } \\
\hline Overbite (mm) & -3.27 & 1.66 & -3.68 & 2.39 & 0.474 \\
\hline Overjet (mm) & 2.72 & 4.94 & 2.23 & 4.66 & 0.711 \\
\hline Interincisal angle $\left(^{\circ}\right)$ & 123.35 & 13.04 & 125.01 & 10.12 & 0.611 \\
\hline
\end{tabular}

${ }^{*}$ Statistically significant at $P<0.05$ 
Table IV. Treatment changes comparison between Group 1 (1-Piece) and Group 2 (4-piece) from T2 to T1 (t test).

\begin{tabular}{|c|c|c|c|c|c|}
\hline \multirow[t]{2}{*}{ Variables } & \multicolumn{2}{|c|}{$\begin{array}{c}\text { Group } 1 \text { (1-Piece) } \\
(\mathrm{N}=29)\end{array}$} & \multicolumn{2}{|c|}{$\begin{array}{c}\text { Group } 2 \text { (4-Piece) } \\
(\mathrm{N}=24)\end{array}$} & \multirow[t]{2}{*}{$\mathbf{P}$} \\
\hline & Mean & s.d & Mean & s.d & \\
\hline \multicolumn{6}{|l|}{ Maxillary component } \\
\hline SNA $\left(^{\circ}\right)$ & 2.37 & 4.82 & 4.75 & 3.64 & 0.052 \\
\hline $\mathrm{CoA}(\mathrm{mm})$ & 2.76 & 4.94 & 3.85 & 8.62 & 0.567 \\
\hline A-Nperp (mm) & 1.55 & 4.32 & 3.83 & 2.96 & $0.032^{*}$ \\
\hline \multicolumn{6}{|c|}{ Mandibular component } \\
\hline SNB $\left(^{\circ}\right)$ & 1.75 & 3.60 & 4.29 & 4.10 & $0.020^{*}$ \\
\hline IMPA $\left({ }^{\circ}\right)$ & 0.99 & 7.38 & -0.05 & 5.77 & 0.574 \\
\hline Pog-NB (mm) & 1.99 & 2.37 & 2.52 & 3.02 & 0.476 \\
\hline GoGn.OP( $\left(^{\circ}\right)$ & -2.80 & 5.25 & -4.18 & 4.86 & 0.327 \\
\hline \multicolumn{6}{|c|}{ Maxillomandibular relationship } \\
\hline ANB $\left({ }^{\circ}\right)$ & 0.62 & 3.65 & 0.41 & 4.72 & 0.857 \\
\hline Wits appraisal (mm) & -0.67 & 6.88 & -0.08 & 5.23 & 0.730 \\
\hline \multicolumn{6}{|l|}{ Growth pattern } \\
\hline $\mathrm{FMA}\left({ }^{\circ}\right)$ & -2.13 & 4.69 & -6.81 & 5.59 & $0.001^{*}$ \\
\hline SN.GoGn( $\left.{ }^{\circ}\right)$ & -2.80 & 5.24 & -7.42 & 5.38 & $0.002^{*}$ \\
\hline SN.PP( $\left(^{\circ}\right)$ & 0.07 & 4.25 & -2.49 & 5.21 & 0.053 \\
\hline SN.OP( $\left(^{\circ}\right)$ & 0.01 & 5.98 & -3.25 & 5.72 & 0.050 \\
\hline \multicolumn{6}{|c|}{ Maxillary dentoalveolar component } \\
\hline Mx1.SN $\left({ }^{\circ}\right)$ & -2.03 & 7.51 & -2.23 & 5.87 & 0.917 \\
\hline Mx1-NA (mm) & -1.14 & 3.75 & -1.68 & 3.42 & 0.590 \\
\hline Mx1Tip-VRP (mm) & 2.63 & 4.13 & 3.99 & 8.26 & 0.440 \\
\hline Mx6-PTV $(\mathrm{mm})$ & 3.11 & 5.38 & 3.86 & 4.19 & 0.580 \\
\hline \multicolumn{6}{|c|}{ Mandibular dentoalveolar component } \\
\hline Md1-MP (mm) & 2.18 & 3.41 & -1.04 & 3.01 & $0.000^{*}$ \\
\hline Md6-MP (mm) & 1.33 & 3.46 & -1.62 & 3.29 & $0.002^{*}$ \\
\hline Md1-Apo (mm) & -0.56 & 3.69 & -2.50 & 2.51 & $0.033^{*}$ \\
\hline \multicolumn{6}{|l|}{ Dental relationships } \\
\hline Overbite $(\mathrm{mm})$ & 4.22 & 1.90 & 4.84 & 2.21 & 0.280 \\
\hline Overjet (mm) & -0.39 & 5.08 & 0.62 & 4.71 & 0.456 \\
\hline Interincisal angle $\left(^{\circ}\right)$ & 3.84 & 10.05 & 9.73 & 7.04 & $0.019^{*}$ \\
\hline
\end{tabular}


Table V. Group 1 (1-Piece) to Group 2 (4-piece) comparison regarding cephalometric variables at $\mathrm{T} 2$ ( $\mathrm{t}$ test).

\begin{tabular}{|c|c|c|c|c|c|}
\hline \multirow[t]{2}{*}{ Variables } & \multicolumn{2}{|c|}{$\begin{array}{c}\text { Group } 1 \text { (1-Piece) } \\
(\mathrm{N}=29)\end{array}$} & \multicolumn{2}{|c|}{$\begin{array}{c}\text { Group } 2 \text { (4-Piece) } \\
(\mathrm{N}=24)\end{array}$} & \multirow[t]{2}{*}{$\mathbf{P}$} \\
\hline & Mean & s.d & Mean & s.d & \\
\hline \multicolumn{6}{|l|}{ Maxillary component } \\
\hline SNA $\left({ }^{\circ}\right)$ & 85.02 & 4.25 & 83.13 & 6.22 & 0.196 \\
\hline $\mathrm{CoA}(\mathrm{mm})$ & 82.83 & 6.12 & 81.64 & 10.81 & 0.618 \\
\hline A-Nperp (mm) & 3.47 & 4.01 & 3.30 & 4.01 & 0.880 \\
\hline \multicolumn{6}{|c|}{ Mandibular component } \\
\hline SNB $\left({ }^{\circ}\right)$ & 80.69 & 5.39 & 80.92 & 5.52 & 0.880 \\
\hline IMPA $\left({ }^{\circ}\right)$ & 88.88 & 9.08 & 83.65 & 6.55 & $0.022^{*}$ \\
\hline Pog-NB (mm) & 3.36 & 2.42 & 4.27 & 2.33 & 0.173 \\
\hline GoGn.OP( $\left(^{\circ}\right)$ & 20.73 & 4.64 & 21.80 & 5.88 & 0.464 \\
\hline \multicolumn{6}{|c|}{ Maxillomandibular relationship } \\
\hline ANB $\left({ }^{\circ}\right)$ & 4.33 & 3.95 & 2.21 & 2.53 & $0.028^{*}$ \\
\hline Wits appraisal (mm) & -2.26 & 3.59 & -2.88 & 3.95 & 0.553 \\
\hline \multicolumn{6}{|l|}{ Growth pattern } \\
\hline $\mathrm{FMA}\left({ }^{\circ}\right)$ & 29.22 & 5.11 & 27.05 & 6.71 & 0.187 \\
\hline SN.GoGn $\left({ }^{\circ}\right)$ & 38.02 & 6.41 & 37.61 & 8.71 & 0.846 \\
\hline SN.PP $\left({ }^{\circ}\right)$ & -0.43 & 4.28 & -1.94 & 5.19 & 0.251 \\
\hline SN.OP( $\left(^{\circ}\right)$ & 17.27 & 6.32 & 15.80 & 6.20 & 0.398 \\
\hline \multicolumn{6}{|c|}{ Maxillary dentoalveolar component } \\
\hline Mx1.SN $\left({ }^{\circ}\right)$ & 105.90 & 8.93 & 104.10 & 7.53 & 0.438 \\
\hline Mx1-NA (mm) & 3.68 & 3.94 & 5.02 & 2.39 & 0.152 \\
\hline Mx1Tip-VRP (mm) & 71.88 & 7.21 & 70.15 & 11.64 & 0.510 \\
\hline Mx6-PTV (mm) & 24.62 & 5.48 & 24.72 & 5.38 & 0.947 \\
\hline \multicolumn{6}{|c|}{ Mandibular dentoalveolar component } \\
\hline Md1-MP (mm) & 41.95 & 4.41 & 39.65 & 5.45 & 0.096 \\
\hline Md6-MP (mm) & 32.58 & 4.32 & 30.07 & 5.32 & 0.063 \\
\hline Md1-Apo (mm) & 3.73 & 2.67 & 2.51 & 1.96 & 0.068 \\
\hline \multicolumn{6}{|l|}{ Dental relationships } \\
\hline Overbite (mm) & 0.94 & 0.58 & 1.08 & 0.72 & 0.432 \\
\hline Overjet (mm) & 2.33 & 0.61 & 2.82 & 1.04 & $0.040^{*}$ \\
\hline Interincisal angle $\left(^{\circ}\right)$ & 127.19 & 8.74 & 134.63 & 7.62 & $0.001^{*}$ \\
\hline
\end{tabular}

*Statistically significant at $P<0.05$ 
Table VI. Posttreatment changes comparison between Group 1 (1-Piece) and Group 2 (4piece) from $T 3$ to $T 2$ ( $t$ test).

\begin{tabular}{|c|c|c|c|c|c|}
\hline \multirow[t]{2}{*}{ Variables } & \multicolumn{2}{|c|}{$\begin{array}{c}\text { Group } 1 \text { (1-Piece) } \\
(\mathrm{N}=29)\end{array}$} & \multicolumn{2}{|c|}{$\begin{array}{c}\text { Group } 2 \text { (4-Piece) } \\
(\mathrm{N}=24)\end{array}$} & \multirow[t]{2}{*}{$\mathbf{P}$} \\
\hline & Mean & s.d & Mean & s.d & \\
\hline \multicolumn{6}{|l|}{ Maxillary component } \\
\hline SNA $\left({ }^{\circ}\right)$ & 0.17 & 2.98 & -0.19 & 3.33 & 0.670 \\
\hline CoA (mm) & 2.68 & 3.29 & 0.41 & 8.25 & 0.179 \\
\hline A-Nperp (mm) & -0.15 & 2.61 & -0.09 & 2.55 & 0.938 \\
\hline \multicolumn{6}{|c|}{ Mandibular component } \\
\hline SNB $\left({ }^{\circ}\right)$ & -0.46 & 2.25 & -0.22 & 2.79 & 0.725 \\
\hline $\operatorname{IMPA}\left({ }^{\circ}\right)$ & 0.83 & 4.88 & 0.42 & 3.62 & 0.732 \\
\hline Pog-NB (mm) & -0.07 & 0.79 & -0.07 & 1.10 & 0.996 \\
\hline GoGn.OP $\left(^{\circ}\right)$ & 0.80 & 3.20 & 0.06 & 2.91 & 0.389 \\
\hline \multicolumn{6}{|c|}{ Maxillomandibular relationship } \\
\hline ANB $\left({ }^{\circ}\right)$ & 0.63 & 2.20 & 0.02 & 1.76 & 0.275 \\
\hline Wits appraisal (mm) & 1.02 & 3.02 & -0.45 & 2.95 & 0.080 \\
\hline \multicolumn{6}{|l|}{ Growth pattern } \\
\hline $\mathrm{FMA}\left({ }^{\circ}\right)$ & 0.97 & 3.17 & 0.64 & 2.27 & 0.675 \\
\hline SN.GoGn( $\left.{ }^{\circ}\right)$ & 0.64 & 3.29 & 0.59 & 3.14 & 0.955 \\
\hline SN.PP( $\left(^{\circ}\right)$ & -0.53 & 2.51 & 0.55 & 3.72 & 0.213 \\
\hline SN.OP( $\left(^{\circ}\right)$ & -0.15 & 2.19 & 0.54 & 4.08 & 0.429 \\
\hline \multicolumn{6}{|c|}{ Maxillary dentoalveolar component } \\
\hline $\mathrm{M} \times 1 . \mathrm{SN}\left({ }^{\circ}\right)$ & 0.15 & 4.77 & -0.65 & 5.00 & 0.554 \\
\hline Mx1-NA (mm) & -0.53 & 2.54 & -0.32 & 2.35 & 0.750 \\
\hline Mx1Tip-VRP (mm) & 1.35 & 2.74 & 0.63 & 8.25 & 0.658 \\
\hline Mx6-PTV (mm) & 1.00 & 2.92 & 0.04 & 3.65 & 0.290 \\
\hline \multicolumn{6}{|c|}{ Mandibular dentoalveolar component } \\
\hline Md1-MP (mm) & 0.44 & 1.31 & 0.52 & 3.48 & 0.908 \\
\hline Md6-MP (mm) & 0.68 & 1.43 & 0.66 & 3.41 & 0.973 \\
\hline Md1-Apo (mm) & -0.08 & 1.69 & 0.11 & 1.20 & 0.632 \\
\hline \multicolumn{6}{|l|}{ Dental relationships } \\
\hline Overbite $(\mathrm{mm})$ & -0.88 & 1.66 & -0.09 & 1.25 & 0.062 \\
\hline Overjet $(\mathrm{mm})$ & 0.22 & 1.93 & -0.43 & 1.30 & 0.162 \\
\hline Interincisal angle $\left(^{\circ}\right)$ & -1.63 & 6.68 & -0.36 & 5.69 & 0.464 \\
\hline
\end{tabular}


Table VII. Group 1 (1-Piece) to Group 2 (4-piece) comparison of cephalometric variables at T3 ( $t$ test) and percentages of patients presenting with clinically significant open bite relapse between groups (chi-square test).

\begin{tabular}{|c|c|c|c|c|c|}
\hline \multirow[t]{2}{*}{ Variables } & \multicolumn{2}{|c|}{$\begin{array}{c}\text { Group } 1 \text { (1-Piece) } \\
(\mathrm{N}=29)\end{array}$} & \multicolumn{2}{|c|}{$\begin{array}{c}\text { Group } 2 \text { (4-Piece) } \\
(\mathrm{N}=24)\end{array}$} & \multirow[t]{2}{*}{$\mathbf{P}$} \\
\hline & Mean & s.d & Mean & s.d & \\
\hline \multicolumn{6}{|l|}{ Maxillary component } \\
\hline SNA $\left({ }^{\circ}\right)$ & 85.21 & 4.35 & 82.77 & 6.40 & 0.106 \\
\hline $\mathrm{CoA}(\mathrm{mm})$ & 85.48 & 5.86 & 82.73 & 9.34 & 0.198 \\
\hline A-Nperp (mm) & 3.29 & 3.07 & 3.09 & 4.80 & 0.851 \\
\hline \multicolumn{6}{|l|}{ Mandibular component } \\
\hline SNB $\left(^{\circ}\right)$ & 80.30 & 5.76 & 80.86 & 6.06 & 0.734 \\
\hline $\operatorname{IMPA}\left({ }^{\circ}\right)$ & 89.70 & 8.92 & 84.14 & 6.16 & $0.012^{*}$ \\
\hline Pog-NB (mm) & 3.30 & 2.48 & 4.24 & 2.06 & 0.143 \\
\hline GoGn.OP( $\left(^{\circ}\right)$ & 21.40 & 5.31 & 21.84 & 5.12 & 0.760 \\
\hline \multicolumn{6}{|l|}{ Maxillomandibular relationship } \\
\hline ANB $\left(^{\circ}\right)$ & 4.90 & 4.48 & 1.90 & 2.51 & $0.005^{*}$ \\
\hline Wits appraisal $(\mathrm{mm})$ & -1.40 & 4.47 & -3.09 & 3.53 & 0.137 \\
\hline \multicolumn{6}{|l|}{ Growth pattern } \\
\hline $\mathrm{FMA}\left({ }^{\circ}\right)$ & 30.14 & 6.02 & 26.80 & 7.33 & 0.074 \\
\hline SN.GoGn $\left({ }^{\circ}\right)$ & 38.56 & 7.46 & 37.32 & 8.16 & 0.565 \\
\hline SN.PP( $\left(^{\circ}\right)$ & -1.07 & 3.36 & -1.73 & 5.21 & 0.577 \\
\hline SN.OP( $\left(^{\circ}\right)$ & 17.16 & 5.92 & 15.48 & 6.24 & 0.322 \\
\hline \multicolumn{6}{|l|}{ Maxillary dentoalveolar component } \\
\hline $\mathrm{M} \times 1 . \mathrm{SN}\left({ }^{\circ}\right)$ & 106.16 & 8.61 & 104.4 & 9.55 & 0.484 \\
\hline $\mathrm{Mx1-NA}(\mathrm{mm})$ & 3.18 & 3.07 & 4.87 & 2.15 & $0.027^{*}$ \\
\hline Mx1Tip-VRP (mm) & 73.22 & 7.36 & 71.02 & 11.69 & 0.408 \\
\hline Mx6-PTV $(\mathrm{mm})$ & 25.66 & 5.29 & 24.99 & 6.04 & 0.668 \\
\hline \multicolumn{6}{|l|}{ Mandibular dentoalveolar component } \\
\hline Md1-MP (mm) & 42.39 & 4.79 & 40.07 & 4.42 & 0.075 \\
\hline Md6-MP (mm) & 33.24 & 4.81 & 30.64 & 4.50 & $0.049^{*}$ \\
\hline Md1-Apo (mm) & 3.65 & 2.68 & 2.54 & 1.83 & 0.089 \\
\hline \multicolumn{6}{|l|}{ Dental relationships } \\
\hline Overbite (mm) & 0.14 & 1.61 & 1.00 & 1.21 & $0.036^{*}$ \\
\hline Overjet $(\mathrm{mm})$ & 2.53 & 1.82 & 2.35 & 1.11 & 0.679 \\
\hline Interincisal angle $\left({ }^{\circ}\right)$ & 125.56 & 11.47 & 134.15 & 8.13 & $0.003^{*}$ \\
\hline Clinically significant stability/relapse & Stability & Relapse & Stability & Relapse & \\
\hline Percentage of patients(\%) & $65.52 \%$ & $34.48 \%$ & $83.33 \%$ & $16.67 \%$ & $0.143 ¥$ \\
\hline Number of patients & $n=19$ & $\mathrm{n}=10$ & $\mathrm{n}=20$ & $\mathrm{n}=4$ & \\
\hline
\end{tabular}

*Statistically significant at $P<0.05$

$¥$ chi-square 
Table VIII. Subgroup 1 (WF) to Group 2 comparability regarding age, treatment time, posttreatment time and cephalometric variables at T1 (t test).

\begin{tabular}{|c|c|c|c|c|c|}
\hline \multirow[t]{2}{*}{ Variables } & \multicolumn{2}{|c|}{$\begin{array}{c}\text { Subgroup1(WF) } \\
\text { (1-Piece) } \\
(\mathrm{N}=17)\end{array}$} & \multicolumn{2}{|c|}{$\begin{array}{c}\text { Group } 2 \\
(4-P i e c e) \\
(\mathrm{N}=24) \\
\end{array}$} & \multirow[t]{2}{*}{$\mathbf{P}$} \\
\hline & Mean & s.d & Mean & s.d & \\
\hline Age (y) & 22.50 & 3.96 & 26.30 & 9.92 & 0.142 \\
\hline Treatment time $(\mathrm{y})$ & 3.20 & 2.42 & 1.27 & 1.23 & $0.001^{*}$ \\
\hline Posttreatment time $(\mathrm{y})$ & 10.65 & 3.62 & 3.71 & 3.20 & $0.000^{*}$ \\
\hline \multicolumn{6}{|l|}{ Maxillary component } \\
\hline $\operatorname{SNA}\left({ }^{\circ}\right)$ & 82.84 & 4.95 & 78.17 & 5.63 & $0.009^{*}$ \\
\hline CoA (mm) & 80.55 & 4.31 & 77.80 & 8.61 & 0.232 \\
\hline A-Nperp (mm) & 2.52 & 4.53 & -0.58 & 4.11 & $0.027^{*}$ \\
\hline \multicolumn{6}{|l|}{ Mandibular component } \\
\hline SNB $\left(^{\circ}\right)$ & 77.71 & 5.52 & 76.52 & 7.95 & 0.595 \\
\hline $\operatorname{IMPA}\left({ }^{\circ}\right)$ & 87.97 & 9.76 & 84.09 & 7.54 & 0.159 \\
\hline Pog-NB (mm) & 1.51 & 1.68 & 1.76 & 1.89 & 0.665 \\
\hline GoGn.OP( $\left(^{\circ}\right)$ & 22.92 & 4.07 & 25.70 & 6.73 & 0.138 \\
\hline \multicolumn{6}{|c|}{ Maxillomandibular relationship } \\
\hline ANB $\left(^{\circ}\right)$ & 5.12 & 6.24 & 1.70 & 5.33 & 0.066 \\
\hline Wits appraisal $(\mathrm{mm})$ & -0.56 & 8.62 & -2.36 & 6.87 & 0.461 \\
\hline \multicolumn{6}{|l|}{ Growth pattern } \\
\hline $\mathrm{FMA}\left({ }^{\circ}\right)$ & 31.75 & 4.64 & 32.90 & 10.07 & 0.666 \\
\hline SN.GoGn $\left({ }^{\circ}\right)$ & 41.68 & 5.36 & 44.22 & 11.48 & 0.402 \\
\hline SN.PP( $\left.{ }^{\circ}\right)$ & 0.09 & 3.28 & 0.29 & 4.36 & 0.873 \\
\hline SN.OP( $\left(^{\circ}\right)$ & 18.75 & 4.70 & 18.52 & 7.67 & 0.911 \\
\hline \multicolumn{6}{|c|}{ Maxillary dentoalveolar component } \\
\hline Mx1.SN $\left(^{\circ}\right)$ & 105.40 & 6.03 & 106.66 & 9.21 & 0.624 \\
\hline Mx1-NA (mm) & 3.78 & 2.81 & 6.65 & 3.16 & $0.004^{*}$ \\
\hline Mx1Tip-VRP (mm) & 68.16 & 8.14 & 65.67 & 10.78 & 0.426 \\
\hline Mx6-PTV (mm) & 20.95 & 4.66 & 21.14 & 5.82 & 0.915 \\
\hline \multicolumn{6}{|c|}{ Mandibular dentoalveolar component } \\
\hline Md1-MP (mm) & 39.38 & 4.11 & 40.35 & 4.64 & 0.493 \\
\hline Md6-MP (mm) & 31.27 & 4.19 & 31.57 & 4.03 & 0.822 \\
\hline Md1-Apo (mm) & 4.07 & 2.59 & 4.81 & 2.41 & 0.350 \\
\hline \multicolumn{6}{|l|}{ Dental relationships } \\
\hline Overbite $(\mathrm{mm})$ & -3.28 & 1.97 & -3.68 & 2.39 & 0.580 \\
\hline Overjet $(\mathrm{mm})$ & 3.68 & 4.98 & 2.23 & 4.66 & 0.344 \\
\hline Interincisal angle $\left(^{\circ}\right)$ & 124.94 & 13.65 & 125.01 & 10.12 & 0.985 \\
\hline
\end{tabular}

*Statistically significant at $P<0.05$ 
Table IX. Treatment changes comparison between Subgroup 1 (WF) to Group 2 (4-piece) from $\mathrm{T} 2$ to $\mathrm{T} 1$ (t test).

\begin{tabular}{|c|c|c|c|c|c|}
\hline \multirow[t]{2}{*}{ Variables } & \multicolumn{2}{|c|}{$\begin{array}{c}\text { Subgroup1(WF) } \\
(1-\mathrm{Piece}) \\
(\mathrm{N}=17)\end{array}$} & \multicolumn{2}{|c|}{$\begin{array}{l}\text { Group } 2 \\
(4-P i e c e) \\
(\mathrm{N}=24)\end{array}$} & \multirow[t]{2}{*}{$\mathbf{P}$} \\
\hline & Mean & s.d & Mean & s.d & \\
\hline \multicolumn{6}{|c|}{ Maxillary component } \\
\hline SNA $\left({ }^{\circ}\right)$ & 2.15 & 4.20 & 4.75 & 3.64 & $0.041^{*}$ \\
\hline CoA (mm) & 1.71 & 4.06 & 3.85 & 8.62 & 0.347 \\
\hline A-Nperp (mm) & 1.26 & 4.29 & 3.83 & 2.96 & $0.028^{*}$ \\
\hline \multicolumn{6}{|c|}{ Mandibular component } \\
\hline SNB $\left({ }^{\circ}\right)$ & 2.00 & 3.19 & 4.29 & 4.10 & 0.062 \\
\hline $\operatorname{IMPA}\left({ }^{\circ}\right)$ & 1.18 & 8.08 & -0.05 & 5.77 & 0.572 \\
\hline Pog-NB (mm) & 2.28 & 2.52 & 2.52 & 3.02 & 0.789 \\
\hline GoGn.OP( $\left(^{\circ}\right)$ & -2.20 & 5.65 & -4.18 & 4.86 & 0.236 \\
\hline \multicolumn{6}{|c|}{ Maxillomandibular relationship } \\
\hline ANB $\left({ }^{\circ}\right)$ & 0.17 & 3.74 & 0.41 & 4.72 & 0.861 \\
\hline Wits appraisal $(\mathrm{mm})$ & -0.67 & 7.97 & -0.08 & 5.23 & 0.775 \\
\hline \multicolumn{6}{|l|}{ Growth pattern } \\
\hline FMA $\left({ }^{\circ}\right)$ & -2.22 & 5.27 & -6.81 & 5.59 & $0.011^{*}$ \\
\hline SN.GoGn( $\left(^{\circ}\right)$ & -3.05 & 5.68 & -7.42 & 5.38 & $0.015^{*}$ \\
\hline SN.PP( $\left(^{\circ}\right)$ & -1.42 & 3.73 & -2.49 & 5.21 & 0.475 \\
\hline SN.OP( $\left(^{\circ}\right)$ & -0.80 & 6.89 & -3.25 & 5.72 & 0.221 \\
\hline \multicolumn{6}{|c|}{ Maxillary dentoalveolar component } \\
\hline $\mathrm{M} \times 1 . \mathrm{SN}\left({ }^{\circ}\right)$ & -1.26 & 8.07 & -2.23 & 5.87 & 0.658 \\
\hline Mx1-NA (mm) & -0.38 & 3.47 & -1.68 & 3.42 & 0.242 \\
\hline Mx1Tip-VRP (mm) & 2.47 & 4.23 & 3.99 & 8.26 & 0.492 \\
\hline Mx6-PTV (mm) & 3.35 & 6.14 & 3.86 & 4.19 & 0.754 \\
\hline \multicolumn{6}{|c|}{ Mandibular dentoalveolar component } \\
\hline Md1-MP (mm) & 1.72 & 2.49 & -1.04 & 3.01 & $0.003^{*}$ \\
\hline Md6-MP (mm) & 0.84 & 3.01 & -1.62 & 3.29 & $0.019^{*}$ \\
\hline Md1-Apo (mm) & -0.60 & 4.19 & -2.50 & 2.51 & 0.077 \\
\hline \multicolumn{6}{|l|}{ Dental relationships } \\
\hline Overbite $(\mathrm{mm})$ & 4.17 & 1.71 & 4.84 & 2.21 & 0.306 \\
\hline Overjet (mm) & -0.12 & 5.51 & 0.62 & 4.71 & 0.643 \\
\hline Interincisal angle $\left(^{\circ}\right)$ & 3.11 & 9.88 & 9.73 & 7.04 & $0.016^{*}$ \\
\hline
\end{tabular}

*Statistically significant at $P<0.05$ 
Table X. Subgroup 1 (WF) to Group 2 (4-piece) comparison regarding cephalometric variables at $\mathrm{T} 2$ ( $\mathrm{t}$ test).

\begin{tabular}{|c|c|c|c|c|c|}
\hline \multirow[t]{2}{*}{ Variables } & \multicolumn{2}{|c|}{$\begin{array}{c}\text { Subgroup1(WF) } \\
\text { (1-Piece) } \\
(\mathrm{N}=17)\end{array}$} & \multicolumn{2}{|c|}{$\begin{array}{c}\text { Group } 2 \\
\text { (4-Piece) } \\
(\mathrm{N}=24)\end{array}$} & \multirow[t]{2}{*}{$\mathbf{P}$} \\
\hline & Mean & s.d & Mean & s.d & \\
\hline \multicolumn{6}{|l|}{ Maxillary component } \\
\hline SNA $\left({ }^{\circ}\right)$ & 84.89 & 4.43 & 83.13 & 6.22 & 0.324 \\
\hline CoA (mm) & 82.66 & 6.86 & 81.64 & 10.81 & 0.734 \\
\hline A-Nperp (mm) & 3.17 & 4.31 & 3.30 & 4.01 & 0.919 \\
\hline \multicolumn{6}{|c|}{ Mandibular component } \\
\hline SNB $\left(^{\circ}\right)$ & 79.75 & 5.96 & 80.92 & 5.52 & 0.524 \\
\hline $\operatorname{IMPA}\left({ }^{\circ}\right)$ & 88.80 & 9.08 & 83.65 & 6.55 & $0.041^{*}$ \\
\hline Pog-NB (mm) & 2.77 & 2.79 & 4.27 & 2.33 & 0.068 \\
\hline GoGn.OP( $\left(^{\circ}\right)$ & 21.12 & 3.71 & 21.80 & 5.88 & 0.676 \\
\hline \multicolumn{6}{|c|}{ Maxillomandibular relationship } \\
\hline ANB $\left(^{\circ}\right)$ & 5.13 & 4.37 & 2.21 & 2.53 & $0.010^{*}$ \\
\hline Wits appraisal (mm) & -1.95 & 4.09 & -2.88 & 3.95 & 0.469 \\
\hline \multicolumn{6}{|l|}{ Growth pattern } \\
\hline $\mathrm{FMA}\left({ }^{\circ}\right)$ & 31.15 & 4.61 & 27.05 & 6.71 & $0.035^{*}$ \\
\hline SN.GoGn( $\left(^{\circ}\right)$ & 39.73 & 6.88 & 37.61 & 8.71 & 0.409 \\
\hline SN.PP( $\left(^{\circ}\right)$ & -0.61 & 4.63 & $-1,94$ & 5.19 & 0.406 \\
\hline SN.OP( $\left(^{\circ}\right)$ & 18.60 & 6.33 & 15.80 & 6.20 & 0.166 \\
\hline \multicolumn{6}{|c|}{ Maxillary dentoalveolar component } \\
\hline Mx1.SN $\left({ }^{\circ}\right)$ & 104.82 & 8.97 & 104.10 & 7.53 & 0.783 \\
\hline Mx1-NA (mm) & 3.12 & 3.83 & 5.02 & 2.39 & 0.057 \\
\hline Mx1Tip-VRP (mm) & 70.60 & 8.41 & 70.15 & 11.64 & 0.894 \\
\hline Mx6-PTV $(\mathrm{mm})$ & 21.94 & 4.94 & 24.72 & 5.38 & 0.100 \\
\hline \multicolumn{6}{|c|}{ Mandibular dentoalveolar component } \\
\hline Md1-MP (mm) & 42.04 & 4.50 & 39.65 & 5.45 & 0.146 \\
\hline Md6-MP (mm) & 32.40 & 4.02 & 30.07 & 5.32 & 0.136 \\
\hline Md1-Apo (mm) & 4.00 & 2.72 & 2.51 & 1.96 & $0.048^{*}$ \\
\hline \multicolumn{6}{|l|}{ Dental relationships } \\
\hline Overbite (mm) & 1.04 & 0.65 & 1.08 & 0.72 & 0.836 \\
\hline Overjet $(\mathrm{mm})$ & 2.40 & 0.57 & 2.82 & 1.04 & 0.141 \\
\hline Interincisal angle $\left(^{\circ}\right)$ & 126.64 & 9.17 & 134.63 & 7.62 & $0.004^{*}$ \\
\hline
\end{tabular}

*Statistically significant at $P<0.05$ 
Table XI. Posttreatment changes comparison between Subgroup 1 (WF) and Group 2 (4piece) from T3 to T2 (t test).

\begin{tabular}{|c|c|c|c|c|c|}
\hline \multirow[t]{2}{*}{ Variables } & \multicolumn{2}{|c|}{$\begin{array}{c}\text { Subgroup1(WF) } \\
\text { (1-Piece) } \\
(\mathrm{N}=17)\end{array}$} & \multicolumn{2}{|c|}{$\begin{array}{c}\text { Group } 2 \\
(4-P i e c e) \\
(\mathrm{N}=24) \\
\end{array}$} & \multirow[t]{2}{*}{$\mathbf{P}$} \\
\hline & Mean & s.d & Mean & s.d & \\
\hline \multicolumn{6}{|l|}{ Maxillary component } \\
\hline SNA $\left(^{\circ}\right)$ & 0.09 & 3.13 & -0.19 & 3.33 & 0.779 \\
\hline $\mathrm{CoA}(\mathrm{mm})$ & 3.67 & 3.34 & 0.41 & 8.25 & 0.133 \\
\hline A-Nperp (mm) & 0.50 & 2.31 & -0.09 & 2.55 & 0.445 \\
\hline \multicolumn{6}{|c|}{ Mandibular component } \\
\hline SNB $\left({ }^{\circ}\right)$ & -0.74 & 2.19 & -0.22 & 2.79 & 0.521 \\
\hline $\operatorname{IMPA}\left({ }^{\circ}\right)$ & 0.92 & 6.01 & 0.42 & 3.62 & 0.739 \\
\hline Pog-NB (mm) & -0.28 & 0.94 & -0.07 & 1.10 & 0.532 \\
\hline GoGn.OP( $\left(^{\circ}\right)$ & 1.51 & 3.62 & 0.06 & 2.91 & 0.164 \\
\hline \multicolumn{6}{|c|}{ Maxillomandibular relationship } \\
\hline ANB $\left(^{\circ}\right)$ & 0.84 & 2.46 & 0.02 & 1.76 & 0.223 \\
\hline Wits appraisal (mm) & 1.52 & 3.28 & -0.45 & 2.95 & 0.050 \\
\hline \multicolumn{6}{|l|}{ Growth pattern } \\
\hline $\mathrm{FMA}\left({ }^{\circ}\right)$ & 1.05 & 3.34 & 0.64 & 2.27 & 0.645 \\
\hline SN.GoGn( $\left.{ }^{\circ}\right)$ & 1.45 & 3.60 & 0.59 & 3.14 & 0.418 \\
\hline SN.PP $\left({ }^{\circ}\right)$ & -0.12 & 2.09 & 0.55 & 3.72 & 0.505 \\
\hline SN.OP( $\left(^{\circ}\right)$ & -0.04 & 2.26 & 0.54 & 4.08 & 0.595 \\
\hline \multicolumn{6}{|c|}{ Maxillary dentoalveolar component } \\
\hline Mx1.SN $\left(^{\circ}\right)$ & -0.47 & 4.86 & -0.65 & 5.00 & 0.909 \\
\hline Mx1-NA (mm) & -0.87 & 2.66 & -0.32 & 2.35 & 0.484 \\
\hline Mx1Tip-VRP (mm) & 1.35 & 2.94 & 0.63 & 8.25 & 0.731 \\
\hline Mx6-PTV $(\mathrm{mm})$ & 1.08 & 3.33 & 0.04 & 3.65 & 0.358 \\
\hline \multicolumn{6}{|c|}{ Mandibular dentoalveolar component } \\
\hline Md1-MP (mm) & 0.67 & 1.37 & 0.52 & 3.48 & 0.869 \\
\hline Md6-MP (mm) & 0.56 & 1.65 & 0.66 & 3.41 & 0.909 \\
\hline Md1-Apo (mm) & -0.04 & 1.99 & 0.11 & 1.20 & 0.761 \\
\hline \multicolumn{6}{|l|}{ Dental relationships } \\
\hline Overbite $(\mathrm{mm})$ & -0.92 & 1.84 & -0.09 & 1.25 & 0.095 \\
\hline Overjet (mm) & 0.12 & 2.26 & -0.43 & 1.30 & 0.325 \\
\hline Interincisal angle $\left(^{\circ}\right)$ & -1.94 & 8.33 & -0.36 & 5.69 & 0.476 \\
\hline
\end{tabular}


Table XII. Subgroup 1 (WF) to Group 2 (4-piece) comparison of cephalometric variables at T3 ( $t$ test) and percentages of patients presenting with clinically significant open bite relapse between groups (chi-square test).

\begin{tabular}{|c|c|c|c|c|c|}
\hline \multirow[t]{2}{*}{ Variables } & \multicolumn{2}{|c|}{$\begin{array}{c}\text { Subgroup1(WF) } \\
\text { (1-Piece) } \\
(\mathrm{N}=17)\end{array}$} & \multicolumn{2}{|c|}{$\begin{array}{c}\text { Group } 2 \\
\text { (4-Piece) } \\
(\mathrm{N}=24) \\
\end{array}$} & \multirow[t]{2}{*}{$\mathbf{P}$} \\
\hline & Mean & s.d & Mean & s.d & \\
\hline \multicolumn{6}{|l|}{ Maxillary component } \\
\hline SNA $\left({ }^{\circ}\right)$ & 84.99 & 4.65 & 82.77 & 6.40 & 0.231 \\
\hline CoA (mm) & 86.14 & 6.32 & 82.73 & 9.34 & 0.199 \\
\hline A-Nperp (mm) & 3.17 & 2.95 & 3.09 & 4.80 & 0.948 \\
\hline \multicolumn{6}{|l|}{ Mandibular component } \\
\hline SNB $\left(^{\circ}\right)$ & 79.04 & 5.99 & 80.86 & 6.06 & 0.348 \\
\hline IMPA $\left({ }^{\circ}\right)$ & 89.21 & 9.91 & 84.14 & 6.16 & 0.050 \\
\hline Pog-NB (mm) & 2.71 & 2.74 & 4.24 & 2.06 & $0.048^{*}$ \\
\hline GoGn.OP( $\left(^{\circ}\right)$ & 22.42 & 4.70 & 21.84 & 5.12 & 0.713 \\
\hline \multicolumn{6}{|c|}{ Maxillomandibular relationship } \\
\hline ANB $\left(^{\circ}\right)$ & 5.94 & 4.87 & 1.90 & 2.51 & $0.001^{*}$ \\
\hline Wits appraisal (mm) & -0.60 & 5.02 & -3.09 & 3.53 & 0.069 \\
\hline \multicolumn{6}{|l|}{ Growth pattern } \\
\hline $\mathrm{FMA}\left({ }^{\circ}\right)$ & 32.34 & 5.60 & 26.80 & 7.33 & $0.012^{*}$ \\
\hline SN.GoGn( $\left(^{\circ}\right)$ & 40.85 & 7.45 & 37.32 & 8.16 & 0.165 \\
\hline SN.PP( $\left(^{\circ}\right)$ & -1.46 & 3.29 & -1.73 & 5.21 & 0.850 \\
\hline SN.OP( $\left(^{\circ}\right)$ & 18.41 & 5.32 & 15.48 & 6.24 & 0.124 \\
\hline \multicolumn{6}{|c|}{ Maxillary dentoalveolar component } \\
\hline Mx1.SN $\left(^{\circ}\right)$ & 104.79 & 8.14 & 104.40 & 9.55 & 0.892 \\
\hline Mx1-NA (mm) & 2.16 & 3.11 & 4.87 & 2.15 & $0.002^{*}$ \\
\hline Mx1Tip-VRP (mm) & 72.18 & 8.47 & 71.02 & 11.69 & 0.727 \\
\hline Mx6-PTV (mm) & 23.54 & 5.06 & 24.99 & 6.04 & 0.423 \\
\hline \multicolumn{6}{|c|}{ Mandibular dentoalveolar component } \\
\hline Md1-MP (mm) & 42.64 & 4.67 & 40.07 & 4.42 & 0.081 \\
\hline Md6-MP (mm) & 33.21 & 4.62 & 30.64 & 4.50 & 0.082 \\
\hline Md1-Apo (mm) & 3.80 & 3.14 & 2.54 & 1.83 & 0.114 \\
\hline \multicolumn{6}{|l|}{ Dental relationships } \\
\hline Overbite (mm) & -0.18 & 1.87 & 1.00 & 1.21 & $0.018^{*}$ \\
\hline Overjet $(\mathrm{mm})$ & 2.52 & 2.23 & 2.35 & 1.11 & 0.750 \\
\hline Interincisal angle $\left({ }^{\circ}\right)$ & 125.14 & 13.27 & 134.15 & 8.13 & $0.010^{*}$ \\
\hline $\begin{array}{c}\text { Clinically significant } \\
\text { relapse } \\
\text { Percentage of patients } \\
(\%) \\
\text { Number of patients }\end{array}$ & $\begin{array}{l}\text { Stability } \\
\begin{array}{c}52.94 \% \\
n=9\end{array}\end{array}$ & $\begin{array}{l}\text { Relapse } \\
\begin{array}{c}47.06 \% \\
n=8\end{array}\end{array}$ & $\begin{array}{l}\text { Stability } \\
83.33 \% \\
n=20\end{array}$ & $\begin{array}{c}\text { Relapse } \\
\begin{array}{c}16.67 \% \\
n=4\end{array}\end{array}$ & $0.035 \#^{*}$ \\
\hline $\begin{array}{l}\text { *Statistically significant a } \\
\text { ¥chi-square }\end{array}$ & & & & & \\
\hline
\end{tabular}


Table XIII. Subgroup 2 (RIF) to Group 2 comparability regarding age, treatment time, posttreatment time and cephalometric variables at T1 (t test).

\begin{tabular}{|c|c|c|c|c|c|}
\hline \multirow[t]{2}{*}{ Variables } & \multicolumn{2}{|c|}{$\begin{array}{c}\text { Subgroup } 2 \text { (RIF) } \\
\text { (1-Piece) } \\
(\mathrm{N}=12)\end{array}$} & \multicolumn{2}{|c|}{$\begin{array}{c}\text { Group } 2 \\
\text { (4-Piece) } \\
(\mathrm{N}=24)\end{array}$} & \multirow[t]{2}{*}{$\mathbf{P}$} \\
\hline & Mean & s.d & Mean & s.d & \\
\hline Age (y) & 24.25 & 7.78 & 26.30 & 9.92 & 0.534 \\
\hline Treatment time $(\mathrm{y})$ & 4.02 & 2.78 & 1.27 & 1.23 & $0.000^{*}$ \\
\hline Posttreatment time (y) & 3.61 & 1.42 & 3.71 & 3.20 & 0.918 \\
\hline \multicolumn{6}{|l|}{ Maxillary component } \\
\hline SNA $\left(^{\circ}\right)$ & 82.37 & 4.43 & 78.17 & 5.63 & $0.030^{*}$ \\
\hline $\mathrm{CoA}(\mathrm{mm})$ & 79.35 & 3.16 & 77.80 & 8.61 & 0.551 \\
\hline A-Nperp (mm) & 1.05 & 4.28 & -0.58 & 4.11 & 0.272 \\
\hline \multicolumn{6}{|l|}{ Mandibular component } \\
\hline SNB $\left(^{\circ}\right)$ & 80.65 & 5.19 & 76.52 & 7.95 & 0.112 \\
\hline IMPA $\left({ }^{\circ}\right)$ & 87.78 & 9.66 & 84.09 & 7.54 & 0.216 \\
\hline Pog-NB (mm) & 1.17 & 1.85 & 1.76 & 1.89 & 0.383 \\
\hline GoGn.OP( $\left(^{\circ}\right)$ & 24.40 & 6.03 & 25.70 & 6.73 & 0.575 \\
\hline \multicolumn{6}{|c|}{ Maxillomandibular relationship } \\
\hline ANB $\left({ }^{\circ}\right)$ & 1.70 & 5.03 & 1.70 & 5.33 & 0.998 \\
\hline Wits appraisal $(\mathrm{mm})$ & -3.05 & 7.28 & -2.36 & 6.87 & 0.784 \\
\hline \multicolumn{6}{|l|}{ Growth pattern } \\
\hline $\mathrm{FMA}\left({ }^{\circ}\right)$ & 30.77 & 5.41 & 32.90 & 10.07 & 0.501 \\
\hline SN.GoGn $\left({ }^{\circ}\right)$ & 39.60 & 6.45 & 44.22 & 11.48 & 0.206 \\
\hline SN.PP $\left({ }^{\circ}\right)$ & -1.35 & 3.72 & 0.29 & 4.36 & 0.269 \\
\hline SN.OP( $\left(^{\circ}\right)$ & 15.19 & 3.08 & 18.52 & 7.67 & 0.159 \\
\hline \multicolumn{6}{|c|}{ Maxillary dentoalveolar component } \\
\hline Mx1.SN $\left({ }^{\circ}\right)$ & 111.53 & 8.36 & 106.66 & 9.21 & 0.133 \\
\hline Mx1-NA (mm) & 6.32 & 3.62 & 6.65 & 3.16 & 0.778 \\
\hline Mx1Tip-VRP (mm) & 70.80 & 4.78 & 65.67 & 10.78 & 0.126 \\
\hline Mx6-PTV $(\mathrm{mm})$ & 22.27 & 6.18 & 21.14 & 5.82 & 0.593 \\
\hline \multicolumn{6}{|c|}{ Mandibular dentoalveolar component } \\
\hline Md1-MP (mm) & 40.31 & 3.88 & 40.35 & 4.64 & 0.980 \\
\hline Md6-MP (mm) & 31.21 & 2.54 & 31.57 & 4.03 & 0.783 \\
\hline Md1-Apo $(\mathrm{mm})$ & 4.64 & 3.14 & 4.81 & 2.41 & 0.854 \\
\hline \multicolumn{6}{|l|}{ Dental relationships } \\
\hline Overbite $(\mathrm{mm})$ & -3.26 & 1.18 & -3.68 & 2.39 & 0.575 \\
\hline Overjet $(\mathrm{mm})$ & 1.36 & 4.75 & 2.23 & 4.66 & 0.604 \\
\hline Interincisal angle $\left({ }^{\circ}\right)$ & 121.09 & 12.35 & 125.01 & 10.12 & 0.315 \\
\hline
\end{tabular}

*Statistically significant at $P<0.05$ 
Table XIV. Treatment changes comparison between Subgroup 2 (RIF) to Group 2 (4-piece) from $\mathrm{T} 2$ to $\mathrm{T} 1$ ( $\mathrm{t}$ test).

\begin{tabular}{|c|c|c|c|c|c|}
\hline \multirow[t]{2}{*}{ Variables } & \multicolumn{2}{|c|}{$\begin{array}{c}\text { Subgroup } 2 \text { (RIF) } \\
(1-\mathrm{Piece}) \\
(\mathrm{N}=12)\end{array}$} & \multicolumn{2}{|c|}{$\begin{array}{l}\text { Group } 2 \\
(4-P i e c e) \\
(\mathrm{N}=24)\end{array}$} & \multirow[t]{2}{*}{$\mathbf{P}$} \\
\hline & Mean & s.d & Mean & s.d & \\
\hline \multicolumn{6}{|l|}{ Maxillary component } \\
\hline SNA $\left({ }^{\circ}\right)$ & 2.68 & 5.78 & 4.75 & 3.64 & 0.060 \\
\hline CoA (mm) & 4.26 & 5.82 & 3.85 & 8.62 & 0.883 \\
\hline A-Nperp (mm) & 1.95 & 4.51 & 3.83 & 2.96 & 0.143 \\
\hline \multicolumn{6}{|c|}{ Mandibular component } \\
\hline SNB $\left({ }^{\circ}\right)$ & 1.40 & 4.24 & 4.29 & 4.10 & 0.057 \\
\hline $\operatorname{IMPA}\left({ }^{\circ}\right)$ & 0.73 & 6.59 & -0.05 & 5.77 & 0.716 \\
\hline Pog-NB (mm) & 1.58 & 2.19 & 2.52 & 3.02 & 0.343 \\
\hline GoGn.OP( $\left(^{\circ}\right)$ & -3.64 & 4.75 & -4.18 & 4.86 & 0.751 \\
\hline \multicolumn{6}{|c|}{ Maxillomandibular relationship } \\
\hline ANB $\left(^{\circ}\right)$ & 1.25 & 3.56 & 0.41 & 4.72 & 0.588 \\
\hline Wits appraisal (mm) & -0.67 & 5.28 & -0.08 & 5.23 & 0.751 \\
\hline \multicolumn{6}{|l|}{ Growth pattern } \\
\hline $\mathrm{FMA}\left({ }^{\circ}\right)$ & -1.99 & 3.94 & -6.81 & 5.59 & $0.011^{*}$ \\
\hline SN.GoGn( $\left(^{\circ}\right)$ & -2.51 & 4.77 & -7.42 & 5.38 & $0.011^{*}$ \\
\hline SN.PP( $\left(^{\circ}\right)$ & 2.20 & 4.15 & -2.49 & 5.21 & $0.010^{*}$ \\
\hline SN.OP( $\left(^{\circ}\right)$ & 1.12 & 4.42 & -3.25 & 5.72 & $0.026^{*}$ \\
\hline \multicolumn{6}{|c|}{ Maxillary dentoalveolar component } \\
\hline Mx1.SN $\left({ }^{\circ}\right)$ & -3.13 & 6.84 & -2.23 & 5.87 & 0.684 \\
\hline Mx1-NA (mm) & -2.22 & 4.01 & -1.68 & 3.42 & 0.677 \\
\hline Mx1Tip-VRP (mm) & 2.85 & 4.16 & 3.99 & 8.26 & 0.656 \\
\hline Mx6-PTV (mm) & 2.77 & 4.34 & 3.86 & 4.19 & 0.471 \\
\hline \multicolumn{6}{|c|}{ Mandibular dentoalveolar component } \\
\hline Md1-MP (mm) & 2.83 & 4.44 & -1.04 & 3.01 & $0.003^{*}$ \\
\hline Md6-MP (mm) & 2.01 & 4.06 & -1.62 & 3.29 & $0.006^{*}$ \\
\hline Md1-Apo (mm) & -0.51 & 3.02 & -2.50 & 2.51 & $0.043^{*}$ \\
\hline \multicolumn{6}{|l|}{ Dental relationships } \\
\hline Overbite (mm) & 4.29 & 2.22 & 4.84 & 2.21 & 0.487 \\
\hline Overjet $(\mathrm{mm})$ & -0.77 & 4.61 & 0.62 & 4.71 & 0.403 \\
\hline Interincisal angle $\left(^{\circ}\right)$ & 4.87 & 10.64 & 9.73 & 7.04 & 0.110 \\
\hline
\end{tabular}

*Statistically significant at $P<0.05$ 
Table XV. Subgroup 2 (RIF) to Group 2 (4-piece) comparison regarding cephalometric variables at $\mathrm{T} 2$ ( $\mathrm{t}$ test).

\begin{tabular}{|c|c|c|c|c|c|}
\hline \multirow[t]{2}{*}{ Variables } & \multicolumn{2}{|c|}{$\begin{array}{c}\text { Subgroup } 2 \text { (RIF) } \\
(1-\mathrm{Piece}) \\
(\mathrm{N}=12)\end{array}$} & \multicolumn{2}{|c|}{$\begin{array}{l}\text { Group } 2 \\
(4-P i e c e) \\
(\mathrm{N}=24)\end{array}$} & \multirow[t]{2}{*}{$\mathbf{P}$} \\
\hline & Mean & s.d & Mean & s.d & \\
\hline \multicolumn{6}{|c|}{ Maxillary component } \\
\hline SNA $\left({ }^{\circ}\right)$ & 85.20 & 4.17 & 83.13 & 6.22 & 0.305 \\
\hline CoA (mm) & 83.06 & 5.18 & 81.64 & 10.81 & 0.670 \\
\hline A-Nperp (mm) & 3.90 & 3.68 & 3.30 & 4.01 & 0.669 \\
\hline \multicolumn{6}{|c|}{ Mandibular component } \\
\hline SNB $\left({ }^{\circ}\right)$ & 82.01 & 4.36 & 80.92 & 5.52 & 0.553 \\
\hline $\operatorname{IMPA}\left({ }^{\circ}\right)$ & 89.01 & 9.50 & 83.65 & 6.55 & 0.055 \\
\hline Pog-NB (mm) & 4.21 & 1.51 & 4.27 & 2.33 & 0.933 \\
\hline GoGn.OP( $\left(^{\circ}\right)$ & 20.19 & 5.86 & 21.80 & 5.88 & 0.443 \\
\hline \multicolumn{6}{|c|}{ Maxillomandibular relationship } \\
\hline ANB $\left({ }^{\circ}\right)$ & 3.19 & 3.10 & 2.21 & 2.53 & 0.319 \\
\hline Wits appraisal $(\mathrm{mm})$ & -2.70 & 2.85 & -2.88 & 3.95 & 0.889 \\
\hline \multicolumn{6}{|l|}{ Growth pattern } \\
\hline FMA $\left({ }^{\circ}\right)$ & 26.48 & 4.66 & 27.05 & 6.71 & 0.795 \\
\hline SN.GoGn( $\left(^{\circ}\right)$ & 35.59 & 4.99 & 37.61 & 8.71 & 0.462 \\
\hline SN.PP( $\left(^{\circ}\right)$ & -0.16 & 3.90 & $-1,94$ & 5.19 & 0.304 \\
\hline SN.OP( $\left(^{\circ}\right)$ & 15.40 & 6.06 & 15.80 & 6.20 & 0.853 \\
\hline \multicolumn{6}{|c|}{ Maxillary dentoalveolar component } \\
\hline $\mathrm{M} \times 1 . \mathrm{SN}\left({ }^{\circ}\right)$ & 107.43 & 9.02 & 104.10 & 7.53 & 0.250 \\
\hline Mx1-NA (mm) & 4.48 & 4.12 & 5.02 & 2.39 & 0.620 \\
\hline Mx1Tip-VRP (mm) & 73.71 & 4.83 & 70.15 & 11.64 & 0.319 \\
\hline Mx6-PTV (mm) & 28.40 & 3.79 & 24.72 & 5.38 & $0.041^{*}$ \\
\hline \multicolumn{6}{|c|}{ Mandibular dentoalveolar component } \\
\hline Md1-MP (mm) & 41.82 & 4.48 & 39.65 & 5.45 & 0.242 \\
\hline Md6-MP (mm) & 32.84 & 4.89 & 30.07 & 5.32 & 0.140 \\
\hline Md1-Apo (mm) & 3.35 & 2.68 & 2.51 & 1.96 & 0.290 \\
\hline \multicolumn{6}{|l|}{ Dental relationships } \\
\hline Overbite $(\mathrm{mm})$ & 0.80 & 0.44 & 1.08 & 0.72 & 0.233 \\
\hline Overjet (mm) & 2.24 & 0.67 & 2.82 & 1.04 & 0.091 \\
\hline Interincisal angle $\left(^{\circ}\right)$ & 127.96 & 8.44 & 134.63 & 7.62 & $0.022^{*}$ \\
\hline
\end{tabular}

*Statistically significant at $P<0.05$ 
Table XVI. Posttreatment changes comparison between Subgroup 2 (RIF) and Group 2 (4piece) from T3 to T2 ( $t$ test).

\begin{tabular}{|c|c|c|c|c|c|}
\hline \multirow[t]{2}{*}{ Variables } & \multicolumn{2}{|c|}{$\begin{array}{c}\text { Subgroup } 2 \text { (RIF) } \\
\text { (1-Piece) } \\
(\mathrm{N}=12)\end{array}$} & \multicolumn{2}{|c|}{$\begin{array}{c}\text { Group } 2 \\
(4-P i e c e) \\
(\mathrm{N}=24) \\
\end{array}$} & \multirow[t]{2}{*}{$\mathbf{P}$} \\
\hline & Mean & s.d & Mean & s.d & \\
\hline \multicolumn{6}{|l|}{ Maxillary component } \\
\hline SNA $\left({ }^{\circ}\right)$ & 0.29 & 2.88 & -0.19 & 3.33 & 0.668 \\
\hline CoA (mm) & 1.30 & 2.77 & 0.41 & 8.25 & 0.722 \\
\hline A-Nperp (mm) & -1.08 & 2.82 & -0.09 & 2.55 & 0.298 \\
\hline \multicolumn{6}{|c|}{ Mandibular component } \\
\hline SNB $\left({ }^{\circ}\right)$ & -0.06 & 2.38 & -0.22 & 2.79 & 0.871 \\
\hline $\operatorname{IMPA}\left({ }^{\circ}\right)$ & 0.70 & 2.83 & 0.42 & 3.62 & 0.814 \\
\hline Pog-NB (mm) & 0.21 & 0.41 & -0.07 & 1.10 & 0.386 \\
\hline GoGn.OP( $\left(^{\circ}\right)$ & -0.20 & 2.25 & 0.06 & 2.91 & 0.780 \\
\hline \multicolumn{6}{|c|}{ Maxillomandibular relationship } \\
\hline ANB $\left(^{\circ}\right)$ & 0.35 & 1.82 & 0.02 & 1.76 & 0.609 \\
\hline Wits appraisal (mm) & 0.30 & 2.57 & -0.45 & 2.95 & 0.458 \\
\hline \multicolumn{6}{|l|}{ Growth pattern } \\
\hline $\operatorname{FMA}\left({ }^{\circ}\right)$ & 1.05 & 3.34 & 0.64 & 2.27 & 0.645 \\
\hline SN.GoGn( $\left(^{\circ}\right)$ & 1.45 & 3.60 & 0.59 & 3.14 & 0.418 \\
\hline SN.PP( $\left(^{\circ}\right)$ & -0.12 & 2.09 & 0.55 & 3.72 & 0.505 \\
\hline SN.OP( $\left(^{\circ}\right)$ & -0.04 & 2.26 & 0.54 & 4.08 & 0.595 \\
\hline \multicolumn{6}{|c|}{ Maxillary dentoalveolar component } \\
\hline Mx1.SN $\left({ }^{\circ}\right)$ & 1.03 & 4.69 & -0.65 & 5.00 & 0.338 \\
\hline Mx1-NA (mm) & -0.05 & 2.39 & -0.32 & 2.35 & 0.755 \\
\hline Mx1Tip-VRP (mm) & 1.35 & 2.55 & 0.63 & 8.25 & 0.769 \\
\hline Mx6-PTV (mm) & 0.90 & 2.34 & 0.04 & 3.65 & 0.465 \\
\hline \multicolumn{6}{|c|}{ Mandibular dentoalveolar component } \\
\hline Md1-MP (mm) & 0.12 & 1.18 & 0.52 & 3.48 & 0.700 \\
\hline Md6-MP (mm) & 0.86 & 1.10 & 0.66 & 3.41 & 0.845 \\
\hline Md1-Apo (mm) & -0.15 & 1.22 & 0.11 & 1.20 & 0.545 \\
\hline \multicolumn{6}{|l|}{ Dental relationships } \\
\hline Overbite $(\mathrm{mm})$ & -0.82 & 1.44 & -0.09 & 1.25 & 0.127 \\
\hline Overjet $(\mathrm{mm})$ & 0.36 & 1.42 & -0.43 & 1.30 & 0.102 \\
\hline Interincisal angle $\left(^{\circ}\right)$ & -1.20 & 3.50 & -0.36 & 5.69 & 0.643 \\
\hline
\end{tabular}


Table XVII. Subgroup 2 (RIF) to Group 2 (4-piece) comparison of cephalometric variables at T3 ( $t$ test) and percentages of patients presenting with clinically significant open bite relapse between groups (chi-square test).

\begin{tabular}{|c|c|c|c|c|c|}
\hline \multirow[t]{2}{*}{ Variables } & \multicolumn{2}{|c|}{$\begin{array}{c}\text { Subgroup } 2 \text { (RIF) } \\
(1-\mathrm{Piece}) \\
(\mathrm{N}=12)\end{array}$} & \multicolumn{2}{|c|}{$\begin{array}{c}\text { Group } 2 \\
(4-P i e c e) \\
(\mathrm{N}=24)\end{array}$} & \multirow[t]{2}{*}{$\mathbf{P}$} \\
\hline & Mean & s.d & Mean & s.d & \\
\hline \multicolumn{6}{|l|}{ Maxillary component } \\
\hline SNA $\left(^{\circ}\right)$ & 85.51 & 4.06 & 82.77 & 6.40 & 0.186 \\
\hline $\mathrm{CoA}(\mathrm{mm})$ & 84.54 & 5.26 & 82.73 & 9.34 & 0.540 \\
\hline A-Nperp (mm) & 3.46 & 3.35 & 3.09 & 4.80 & 0.810 \\
\hline \multicolumn{6}{|l|}{ Mandibular component } \\
\hline SNB $\left({ }^{\circ}\right)$ & 82.09 & 5.13 & 80.86 & 6.06 & 0.551 \\
\hline $\operatorname{IMPA}\left({ }^{\circ}\right)$ & 90.39 & 7.66 & 84.14 & 6.16 & $0.012^{*}$ \\
\hline Pog-NB (mm) & 4.13 & 1.85 & 4.24 & 2.06 & 0.874 \\
\hline GoGn.OP( $\left.{ }^{\circ}\right)$ & 19.95 & 5.99 & 21.84 & 5.12 & 0.330 \\
\hline \multicolumn{6}{|c|}{ Maxillomandibular relationship } \\
\hline ANB $\left(^{\circ}\right)$ & 3.42 & 3.54 & 1.90 & 2.51 & 0.147 \\
\hline Wits appraisal $(\mathrm{mm})$ & -2.53 & 3.43 & -3.09 & 3.53 & 0.650 \\
\hline \multicolumn{6}{|l|}{ Growth pattern } \\
\hline $\mathrm{FMA}\left({ }^{\circ}\right)$ & 27.01 & 5.33 & 26.80 & 7.33 & 0.928 \\
\hline SN.GoGn( $\left.{ }^{\circ}\right)$ & 35.32 & 6.43 & 37.32 & 8.16 & 0.464 \\
\hline SN.PP $\left({ }^{\circ}\right)$ & -0.51 & 3.52 & -1.73 & 5.21 & 0.470 \\
\hline SN.OP( $\left(^{\circ}\right)$ & 15.38 & 6.48 & 15.48 & 6.24 & 0.963 \\
\hline \multicolumn{6}{|c|}{ Maxillary dentoalveolar component } \\
\hline $\mathrm{M} \times 1 . \mathrm{SN}\left({ }^{\circ}\right)$ & 108.10 & 9.25 & 104.40 & 9.55 & 0.275 \\
\hline Mx1-NA (mm) & 4.62 & 2.46 & 4.87 & 2.15 & 0.756 \\
\hline Mx1Tip-VRP (mm) & 74.68 & 5.43 & 71.02 & 11.69 & 0.312 \\
\hline Mx6-PTV (mm) & 28.66 & 4.16 & 24.99 & 6.04 & 0.067 \\
\hline \multicolumn{6}{|c|}{ Mandibular dentoalveolar component } \\
\hline Md1-MP (mm) & 42.04 & 5.14 & 40.07 & 4.42 & 0.242 \\
\hline Md6-MP (mm) & 33.27 & 5.28 & 30.64 & 4.50 & 0.128 \\
\hline Md1-Apo (mm) & 3.45 & 1.98 & 2.54 & 1.83 & 0.177 \\
\hline \multicolumn{6}{|l|}{ Dental relationships } \\
\hline Overbite $(\mathrm{mm})$ & 0.60 & 1.07 & 1.00 & 1.21 & 0.339 \\
\hline Overjet $(\mathrm{mm})$ & 2.54 & 1.09 & 2.35 & 1.11 & 0.635 \\
\hline Interincisal angle $\left({ }^{\circ}\right)$ & 126.15 & 8.83 & 134.15 & 8.13 & $0.010^{*}$ \\
\hline $\begin{array}{c}\text { Clinically significant } \\
\text { relapse } \\
\text { Percentage of patients } \\
(\%) \\
\text { Number of patients }\end{array}$ & $\begin{array}{c}\text { Stability } \\
83.33 \% \\
n=10\end{array}$ & $\begin{array}{l}\text { Relapse } \\
\begin{array}{c}16.67 \% \\
n=2\end{array}\end{array}$ & $\begin{array}{c}\text { Stability } \\
83.33 \% \\
n=20\end{array}$ & $\begin{array}{l}\text { Relapse } \\
\begin{array}{c}16.67 \% \\
n=4\end{array}\end{array}$ & $0.999 ¥$ \\
\hline $\begin{array}{l}\text { *Statistically significant } \\
¥ \text { chi-square }\end{array}$ & & & & & \\
\hline
\end{tabular}


Table XVIII. Posttreatment time of clinically stable/relapsed subjects' comparison in Group 1 (1-piece) and in Group 2 (4-piece) (t-tests).

\begin{tabular}{|c|c|c|c|c|c|}
\hline Overbite status at T3 & \multicolumn{2}{|c|}{ Clinically stable } & \multicolumn{2}{|c|}{ Clinically relapsed } & P \\
\cline { 2 - 5 } & mean & s.d & mean & sd & \multirow{2}{*}{0.282} \\
\hline $\begin{array}{c}\text { Group 1 (1-piece) } \\
\text { Posttreatment time } \\
\text { (y) }\end{array}$ & 7.07 & 4.58 & 9.01 & 4.45 & \\
\hline $\begin{array}{c}\text { Group 2 (4-piece) } \\
\text { Posttreatment time } \\
(\mathrm{y})\end{array}$ & 4.87 & 2.77 & 2.64 & 0.62 & 0.382 \\
\hline
\end{tabular}




\title{
3.2 ARTICLE 2
}

\section{LONG-TERM STABILITY OF SURGICAL-ORTHODONTIC OPEN BITE} TREATMENT: CLASS II VERSUS CLASS III

\begin{abstract}
Introduction: Few specific studies are comparing Class II to Class III malocclusions on the long-term stability of anterior open bite surgical-orthodontic treatment. Materials and Methods: Lateral cephs obtained from 46 treated patients were divided into two groups: Class II Group (21 patients) and Class III Group (25 patients). Overbite changes were compared at 3-time points: T1 (pretreatment), T2 (posttreatment) and T3 (follow-up) by using t-test. Results: Follow up time mean was 5.75 years ( 2 to 18.83 ys). Both Groups presented a positive overbite mean at T3, however, Class II Group presented with a smaller statistically significant clinical stability $(57.14 \%)$ than Class III Group (88\%). Conclusion: The null hypothesis was rejected, because, the clinical stability of Class II open bite surgical-orthodontic treatment was smaller than in Class III malocclusions.
\end{abstract}




\section{INTRODUCTION}

Open bite treatment demands titanic efforts from the clinicians, firstly because treatment goals are both static and functional, and secondly because results should be maintained in the long-term. When treatment plan involves an surgical-orthodontic approach, patients' expectations usually increase as well as our responsibilities on delivering a stable result.

Long-term stability of skeletal open bites treated using surgical-orthodontic approach has been a controversial issue, for both orthodontists and oral surgeons. Challenges are even greater when anteroposterior discrepancies (Class II and Class III) are present on a vertical dysplasia such as skeletal open bites.

Class II malocclusion can, theoretically, be surgical-orthodontic ly corrected by maxillary and or maxillomandibular advancements, depending on its etiology, severity, and the impact it causes on patients' esthetics and functions ${ }^{1}$. Conversely, Class III malocclusions can be corrected surgical-orthodontic ly by maxillary advancement and or mandible set-back. Correction of high mandibular plane angle malocclusions can be achieved by counter-clockwise or clockwise rotation of the maxillomandibular complex with similar stable results. ${ }^{2-4}$

Open bite stability of Class II malocclusions corrections is referred to be smaller than those of Class III. However just a few specific and, well-designed studies investigated this speculation. ${ }^{5-8}$ The major problems with the current studies are related to sample heterogeneity and short posttreatment follow-up. As a common finding, those studies used to consider 1 or 2-year post-surgery follow-up, which is not an adequate follow-up length. ${ }^{9,10}$ Overbite behavior, which can be influenced by skeletal and dentoalveolar components, should be evaluated at least 2 or 3 years (ideally five years at least) after fixed appliances have been removed. Proffit ${ }^{11}$ established a hierarchy of surgical procedures considering a short-term stability period, based on postsurgical follow-up of 1 year, however, recently an adequation to this parameter was taken into account. ${ }^{12}$

This study aims to test the null hypothesis that there is no difference in the long-term clinical stability of open bite surgical-orthodontic treatment when Class II subjects are compared to Class III. 


\section{MATERIAL AND METHODS}

This study is the second part of research submitted and approved by the institutional review board of Bauru Dental School, University of Sao Paulo, Brazil, under the protocol number: CAAE 71636716.5.0000.5417.

The sample size was calculated based on $80 \%$ test power with a significance level of $5 \%$; a minimum mean difference of $1.43 \mathrm{~mm}$ and a mean standard deviation of $1.6 \mathrm{~mm}$. Hence, 21 subjects in each group were necessary to generate reliable results. Overbite relapse was used according to a previous study. ${ }^{13}$

Inclusion criteria comprised: initial anterior open-bite malocclusion of at least $1 \mathrm{~mm}$, treated orthodontically and surgically, Class II and Class III malocclusions, no previous orthodontic treatment; positive overbite after treatment; comparable retention protocols; facial trauma absence; buccofacial pathology absence, genetic syndromes/ congenital deformities absence.

The sample was divided into two groups:

Group 1: Class II comprised 21 patients (8 male, 13 female) with a mean age of 25.42 years (ranging from 15.08 years to 41.50 years). In only three patients orthognathic surgery was restricted to the maxilla, while the remaining 18 patients were treated by combining 1-piece Le Fort I to BSSO (Bilateral Sagittal Split Osteotomy). Surgical fixation with intraosseous wires was performed in 8 patients (38.09\%), and $13(61.91 \%)$ received rigid internal fixation. Regarding the type of Le Fort I osteotomy, 12 (57.14\%) patients were treated with 1-piece Le Fort I and 9 $(42.86 \%)$ patients with 4-piece Le Fort I osteotomy. Patients in this group were treated by different professional teams, including 6 surgeons, as described: surgeon \# 1 (12 patients), surgeon \# 2 (1 patient), surgeon \# 3 (2 patients), surgeon \# 4 (2 patients), surgeon \# 5 (3 patients) and surgeon \# 6 (1 patient)). The posttreatment follow-up interval began after orthodontic treatment and extended to the last posttreatment patient recall, which had a mean time of 6.34 years (ranging from 2 years to 18.83 years).

Group 2: Class III group comprised 25 patients (15 male, 10 female) with a mean age of 23.08 years (ranging from 14.83 years to 45.25 years). In only five patients orthognathic surgery was restricted to the maxilla, while the remaining 20 patients were treated by combining 1-piece Le Fort I to BSSO (Bilateral Sagittal Split 
Osteotomy). Surgical fixation with intraosseous wires was performed in 6 patients (24\%), and $19(76 \%)$ received rigid internal fixation. Regarding the type of Le Fort I osteotomy, $13(52 \%)$ patients were treated with 1-piece Le Fort I and 12 (48\%) patients with 4-piece Le Fort I osteotomy. Patients in this group were treated by different professional teams, including 6 surgeons, as described: surgeon \# 1 (13 patients), surgeon \# 2 (1 patient), surgeon \# 3 (4 patients), surgeon \# 4 (3 patients), surgeon \# 5 (1 patient) and surgeon \# 6 (3 patients)). The posttreatment follow-up interval began after orthodontic treatment and extended to the last posttreatment patient recall, which had a mean time of 5.26 years (ranging from 2 years to 13.91 years).

\section{Methods}

Lateral cephs were obtained at three time points (T1-pretreatment, T2posttreatment, and T3-long-term follow-up). Several x-ray machines were used and so different magnification factors were present, varying from $7 \%$ to $12.6 \%$. All cephalometric measurements are described in Table I. Cephalometric tracings and magnification corrections were performed with Dolphin $®$ Imaging 11.0 (Dolphin Imaging and Management Solutions, Chatsworth, Calif., EUA).

\section{Error study}

Error study assessment comprised 32 of the 138 lateral cephs which were randomly selected, retraced, and redigitized by the same operator (VLBS) after a 30day interval. Random errors were calculated according to Dahlberg's formula (Se2 = $\left.\sum \mathrm{d} 2 / 2 \mathrm{n}\right),{ }^{14}$ where $\mathrm{Se} 2$ is the error variance and $\mathrm{d}$ is the difference between two determinations of the same variable, and the systematic errors with dependent $t$ tests, at $\mathrm{P}<0.05$. $^{15-17}$

\section{Statistical analyses}

Normal distribution of data in the sample was assessed by applying Kolmogorov-Smirnov tests. All variables showed a normal distribution in the two groups. 
T tests were used to evaluate groups ' comparability regarding age and chisquare test was used to compare the groups regarding sex, and type of fixation distributions.

Intergroup comparison of all variables at T1, T2-T1, T2, T3-T2 and T3 was performed by using $t$ tests.

The Probability of clinical open bite stability was calculated dividing the number of events by the total sample amount and within the subgroups. The percentages of patients with clinically significant relapses in each group were compared with the chi-square test. Results were considered significant at $P<0.05$. All statistical analyses were performed with Statistica software (Statistica for Windows 10.0; StatSoft, Tulsa, Okla.).

\section{RESULTS}

Three variables presented with significant systematic error (Mx1.SN; Mx1-NA; Interincisal angle), requiring caution when taking these measurements into account. Random errors varied within acceptable limits from $0.20 \mathrm{~mm}$ (overbite) to $3.28 \mathrm{~mm}$ (CoA). ${ }^{16}$

Both groups were comparable regarding initial age, treatment time, posttreatment time and sex after t-test and chi-square assessments, respectively (Tables II and III). Groups were comparable regarding the type of fixation distribution (WF and RIF) (Table VIII).

Group 1 (Class II) patients presented with a more retruded mandible, greater mandibular incisors buccal inclination, greater mandibular plane inclination, positive ANB angle and greater overjet than those of Group 2 (Class III) at T1 (Table III).

Regarding treatment changes, both groups presented with SNA angle and ANperp measurement increases (Table IV), however, Group 1 (Class II) showed a smaller increase than Group II (Class III). Groups presented a reduction in the inclination of the mandibular plane and more palatally inclined maxillary incisors. Considering ANB angle, Wits, and overjet measurements, Group 1 (Class II) had a decrease, while Group II (Class III) presented with an increase. Regarding occlusal plane inclination, mandibular molars, and incisors height, Group 1 (Class II) had an 
increase in those measurements, while Group II (Class III) presented with a decrease (Table IV).

At the end of treatment (T2), despite treatment changes, groups tended to maintain their initial cephalometric differences except for overjet measurement which presented very similar values (Table V).

Posttreatment phase changes (T3-T2) were comparable among groups (Table $\mathrm{VI})$.

Groups tended to maintain their initial cephalometric characteristics at T3.

\section{Overbite}

Initial overbite severity was comparable (T1), proportionally corrected with treatment (T2-T1), comparable at the end of treatment (T2), proportionally reduced in the posttreatment phase (T3-T2), and the long-term control (T3) remained positive. However, Group 1 (Class II) presented with smaller clinical overbite stability $(57.14 \%)$ than Class III group (88\%) (Table VII).

\section{DISCUSSION}

The Long-term posttreatment time of 5.75 years after fixed appliance removal present in this sample is an important factor which differentiates, positively, the conclusions of our study, since most of the previous studies considered only 1 or 2 years of postsurgical follow-up time ${ }^{4,8}$. Initial open bite severity was similar among groups allowing a comparable scenario for the open bite stability assessment.

As already expected, groups presented initial cephalometric differences, mainly related to the mandibular component, exposing the antagonistic nature of this two malocclusions. ${ }^{18-20}$ Skeletal Class II characteristics in Group 1 showed that the anteroposterior discrepancy was due to a mandibular retrusion, whereas Group 2 revealed a maxillary retrusion and well-positioned mandible, which, in fact, may be the consequence of the clock-wise mandible rotation direction in the high-angle patients. Because only non-growing patients were selected, the greater vertical growth pattern found in Group 1 than in Group 2 must not have influenced the results. The significant greater ANB angle severity and Wits appraisal means in Class II group than in Class III group, demonstrated the skeletal and dental discrepancy of the sample. Dental compensations, which must be eliminated by presurgical orthodontic preparation, where also demonstrated the greater buccal inclination of mandibular incisors in Class II group and the lingually tipped mandibular incisors in 
Class III group. Moreover, interincisal angle presented with a smaller mean in Class II group than in Class III group (Table III).

Regarding treatment changes, SNA angle and A-Nperp measurements presented a smaller increase in Group 1 than in Group 2, reflecting a logical tendency of promoting a minor maxillary advancement in Class II patients, because the mandible must be advanced to improve the patient profile. And the contrary is, also, true because Class III often requires great maxillary advancements and or mandible set-back. ${ }^{21}$ Maxillary impaction which is usually necessary to correct the divergence between palatal and mandibular planes results in a counter-clockwise rotation of the mandible as our results demonstrate with negative GoGn.OP values for both groups. ${ }^{3}$ The magnitude of overbite correction was almost the same in both groups

At the end of surgical-orthodontic treatment, initial cephalometric characteristics tended to remain among groups. The results of anteroposterior correction in both groups can also be explained by the interaction between ANB, overjet, and maxillary/mandibular incisor inclination at T2. In Class II group, the final ANB angle mean of $5.45^{\circ}$ and the overjet mean of $2.5 \mathrm{~mm}$, by itself, cannot explain sagittal correction. However, the mandibular incisor buccal inclination and the maxillary incisor lingual inclination have compensated for the skeletal discrepancy (Table IV). Conversely, in Class III group, the final ANB mean of $1.63^{\circ}$ with an overjet mean of $2.48 \mathrm{~mm}$ is compensated by mandibular incisor lingual inclination and maxillary incisor buccal inclination. Comparable final overbite means were found between groups, allowing an equal comparison, since initial severity was also comparable. One factor that is, commonly, associated to relapse in surgicalorthodontic treatment of Class II and III open bite patients is the mandibular plane correction. ${ }^{3,4}$ Skeletal changes tend to be counteracted by dentoalveolar alterations, which is known as the compensatory mechanism. ${ }^{22}$ In other words, despite the relapse occurred in the counterclockwise correction of mandibular plane in Class II group, mandibular molars and incisors had an increase in the vertical plane, compensating for the tendency of opening the bite.

Posttreatment changes were not statistically significant among groups but demonstrated that the general relapse tendency follows to the reversion of the 
treatment changes. Interestingly is that, although not statistically significant, Class II overbite mean $(-0.75 \mathrm{~mm})$ decreased three-times more than Class III $(-0.20 \mathrm{~mm})$.

After follow-up period, final overbite means were not statistically significant different among groups, however, Class II group mean $(0.17 \mathrm{~mm})$ was much closer to an open bite (overbite<0 $<\mathrm{mm}$ ) than Class III mean $(0.87 \mathrm{~mm})$.

The evaluation of clinically significant stability, which is the main objective of this study, showed that overall sample presented with a clinically stability rate of $73.91 \%$, corroborating other studies. ${ }^{23,24}$ It is hard to weight if patients went for speech therapist evaluation after treatment since it was not considered for this study.

At the end of posttreatment phase, Class II group (57.14\%) presented with smaller clinically significant stability of overbite than Class III group (88\%). Nine out of 21 patients in Class II group presented a clinical relapse and in Class III group, only 3 out of 25 ended up follow-up period with an overbite smaller than $0 \mathrm{~mm}$. These results are in agreement of several other studies, ${ }^{13,25-27}$ however most of them considered a short-term follow-up period and small sample size, hence our results tends to confirm that Class II surgical-orthodontic treatment is less stable than Class III in open bite patients with stronger evidence. 


\section{CONCLUSIONS}

The null hypothesis was rejected because:

- Clinical stability of Class II malocclusion open bite surgical-orthodontic treatment was significantly smaller than in Class III malocclusions. 


\section{REFERENCES}

1-Fontes AM, Joondeph DR, Bloomquist DS, Greenlee GM, Wallen TR, Huang GJ. Long-term stability of anterior open-bite closure with bilateral sagittal split osteotomy. Am J Orthod Dentofacial Orthop 2012;142:792-800.

2-Goncalves JR, Cassano DS, Wolford LM, Santos-Pinto A, Marquez IM. Postsurgical stability of counterclockwise maxillomandibular advancement surgery: affect of articular disc repositioning. J Oral Maxillofac Surg 2008;66:724-738.

3-Kor HS, Yang HJ, Hwang SJ. Relapse of skeletal class III with anterior open bite after bimaxillary orthognathic surgery depending on maxillary posterior impaction and mandibular counterclockwise rotation. J Craniomaxillofac Surg 2014;42:e230238.

4-Reyneke JP, Bryant RS, Suuronen R, Becker PJ. Postoperative skeletal stability following clockwise and counter-clockwise rotation of the maxillomandibular complex compared to conventional orthognathic treatment. $\mathrm{Br} \mathrm{J}$ Oral Maxillofac Surg 2007;45:56-64.

5-Maia FA, Janson G, Barros SE, Maia NG, Chiqueto K, Nakamura AY. Longterm stability of surgical-orthodontic open-bite correction. Am J Orthod Dentofacial Orthop 2010;138:254 e251-254 e210; discussion 254-256.

6-Bailey LJ, Duong HL, Proffit WR. Surgical Class III treatment: long-term stability and patient perceptions of treatment outcome. Int $\mathrm{J}$ Adult Orthodon Orthognath Surg 1998;13:35-44.

7-Costa F, Robiony M, Sembronio S, Polini F, Politi M. Stability of skeletal Class III malocclusion after combined maxillary and mandibular procedures. Int J Adult Orthodon Orthognath Surg 2001;16:179-192.

8-lannetti G, Fadda MT, Marianetti TM, Terenzi V, Cassoni A. Long-term skeletal stability after surgical correction in Class III open-bite patients: a retrospective study on 40 patients treated with mono-or bimaxillary surgery. J Craniofac Surg 2007;18:350-354.

9-Bailey L, Phillips C, Proffit W, Turvey T. Stability following superior repositioning of the maxilla by Le Fort I osteotomy: five-year follow-up. Int J Adult Orthod Orthognath Surg 1994;9:163-173.

10-Maia FA, Janson G, Barros SE, Maia NG, Chiqueto K, Nakamura AY. Editor's Comment and Q\&A: Long-term stability of surgical-orthodontic open-bite correction. Am J Orthod Dentofac Orthop 2010;138:254-256.

11-Proffit WR, Turvey TA, Phillips C. Orthognathic surgery: a hierarchy of stability. Int J Orthod Orthog Surg 1996;11:191-204. 
12-Proffit WR, White J. Combined surgical-orthodontic treatment: How did it evolve and what are the best practices now? Am J Orthod Dentofacial Orthop 2015;147:205-215.

13- Fischer K, von Konow L, Brattstrom V. Open bite: stability after bimaxillary surgery--2-year treatment outcomes in 58 patients. Eur J Orthod. 2000 Dec;22(6):711-8.

14-Dahlberg G. Statistical methods for medical and biological students. New York: Interscience; 1940.

15 -Houston W. The analysis of errors in orthodontic measurements. Am J Orthod 1983;83.

16-Baumrind S, Frantz RC. The reliability of head film measurements: 1. Landmark identification. Am J Orthod 1971;60.

17-Richardson A. A classification of open bites. Eur J Orthod 1981;3.

18-Ellis E, 3rd, McNamara JA, Jr., Lawrence TM. Components of adult Class II open-bite malocclusion. J Oral Maxillofac Surg 1985;43:92-105.

19-Ellis E, McNamara Jr JA. Components of adult Class III maloclusion. J Oral Maxillofac Surg 1984;42:295-305.

20-Janson G, Laranjeira V, Rizzo M, Garib D. Posterior tooth angulations in patients with anterior open bite and normal occlusion. Am J Orthod Dentofacial Orthop 2016;150:71-77.

21-Epker BN, Fish LC. Surgical-orthodontic correction of open-bite deformity. Am J Orthod 1977;71:278-299.

22-Solow B. The dentoalveolar compensatory mechanism: background and clinical implications. J Orthod 1980;7:145-161.

23-Greenlee GM, Huang GJ, Chen SS, Chen J, Koepsell T, Hujoel P. Stability of treatment for anterior open-bite malocclusion: a meta-analysis. Am J Orthod Dentofacial Orthop 2011;139:154-169.

24-Huang GJ. Long-term stability of anterior open-bitetherapy: A review Semin Orthod: Elsevier; 2002: p. 162-172.

25-Jakobsone G, Stenvik A, Sandvik L, Espeland L. Three-year follow-up of bimaxillary surgery to correct skeletal Class III malocclusion: stability and risk factors for relapse. Am J Orthod Dentofacial Orthop 2011;139:80-89.

26-lannetti G, Fadda MT, Marianetti TM, Terenzi V, Cassoni A. Long-term skeletal stability after surgical correction in Class III open-bite patients: a 
retrospective study on 40 patients treated with mono- or bimaxillary surgery. J Craniofac Surg 2007;18:350-354.

27- Swinnen K, Politis C, Willems G, De Bruyne I, Fieuws S, Heidbuchel K et al. Skeletal and dento-alveolar stability after surgical-orthodontic treatment of anterior open bite: a retrospective study. Eur J Orthod 2001;23:547-557. 
Table I. Cephalometric variables description.

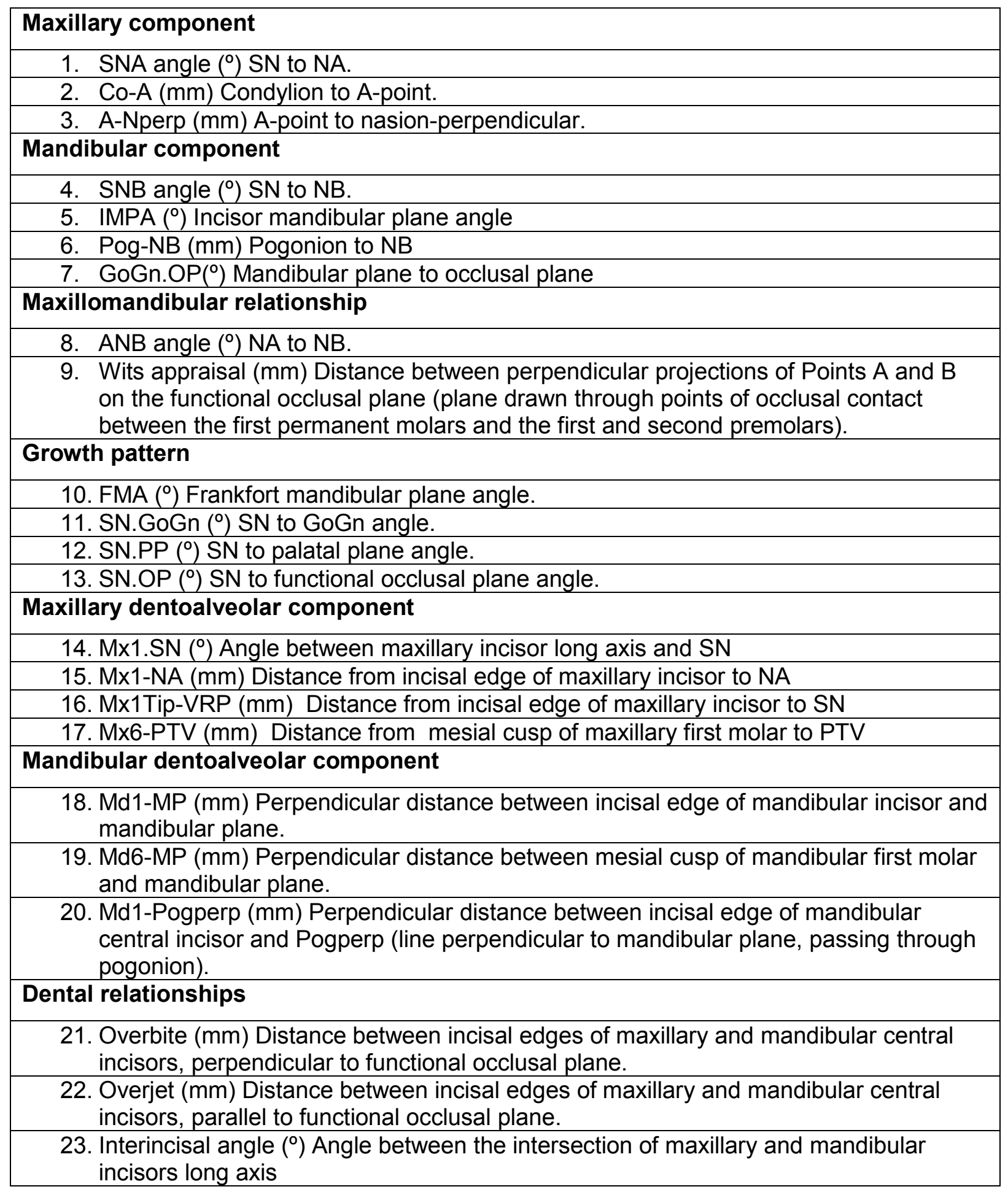


Table II. Intergroup comparability regarding sex (chi-square).

\begin{tabular}{|c|c|c|c|}
\hline Group & Male & Female & Total \\
\hline G1(Class II) & 8 & 13 & 21 \\
\hline G2(Class III) & 15 & 10 & 25 \\
\hline Total & 23 & 23 & 46 \\
\hline \multicolumn{2}{|c|}{$\mathbf{P = 0 . 1 3 8}$} \\
\hline
\end{tabular}


Table III. Group 1 (Class II) to Group 2 (Class III) comparability regarding age, treatment time, posttreatment time and cephalometric variables at $\mathrm{T1}$ (t test).

\begin{tabular}{|c|c|c|c|c|c|}
\hline \multirow[t]{2}{*}{ Variables } & \multicolumn{2}{|c|}{$\begin{array}{c}\text { Group } 1 \text { (Class II) } \\
(\mathrm{N}=21)\end{array}$} & \multicolumn{2}{|c|}{$\begin{array}{c}\text { Group } 2 \text { (Class III) } \\
(\mathrm{N}=25)\end{array}$} & \multirow[t]{2}{*}{$\mathbf{P}$} \\
\hline & Mean & s.d & Mean & s.d & \\
\hline Age (y) & 25.42 & 8.31 & 23.12 & 6.79 & 0.306 \\
\hline Treatment time $(\mathrm{y})$ & 2.74 & 2.79 & 2.19 & 1.86 & 0.424 \\
\hline Posttreatment time $(\mathrm{y})$ & 6.34 & 5.07 & 5.25 & 3.76 & 0.409 \\
\hline \multicolumn{6}{|l|}{ Maxillary component } \\
\hline SNA $\left({ }^{\circ}\right)$ & 80.50 & 4.96 & 79.95 & 5.24 & 0.721 \\
\hline CoA (mm) & 78.09 & 6.71 & 79.07 & 5.93 & 0.600 \\
\hline A-Nperp (mm) & 1.79 & 3.12 & -0.03 & 4.61 & 0.130 \\
\hline \multicolumn{6}{|l|}{ Mandibular component } \\
\hline SNB $\left(^{\circ}\right)$ & 73.78 & 5.74 & 81.05 & 5.89 & $0.000^{*}$ \\
\hline $\operatorname{IMPA}\left({ }^{\circ}\right)$ & 89.84 & 7.47 & 82.15 & 8.88 & $0.002^{*}$ \\
\hline Pog-NB (mm) & 1.06 & 1.49 & 2.14 & 2.08 & 0.053 \\
\hline GoGn.OP( $\left(^{\circ}\right)$ & 26.27 & 6.02 & 22.71 & 5.53 & $0.042^{*}$ \\
\hline \multicolumn{6}{|c|}{ Maxillomandibular relationship } \\
\hline ANB $\left(^{\circ}\right)$ & 6.72 & 4.65 & -1.05 & 4.45 & $0.000^{*}$ \\
\hline Wits appraisal (mm) & 3.74 & 5.72 & -7.64 & 5.14 & $0.000 *$ \\
\hline \multicolumn{6}{|l|}{ Growth pattern } \\
\hline $\mathrm{FMA}\left({ }^{\circ}\right)$ & 34.11 & 6.18 & 30.02 & 8.70 & 0.078 \\
\hline SN.GoGn $\left({ }^{\circ}\right)$ & 45.58 & 8.09 & 40.10 & 9.65 & $0.045^{*}$ \\
\hline SN.PP $\left({ }^{\circ}\right)$ & 0.76 & 3.05 & 0.15 & 4.05 & 0.573 \\
\hline SN.OP( $\left(^{\circ}\right)$ & 19.33 & 5.33 & 17.38 & 6.59 & 0.281 \\
\hline \multicolumn{6}{|c|}{ Maxillary dentoalveolar component } \\
\hline Mx1.SN $\left({ }^{\circ}\right)$ & 105.54 & 6.29 & 108.67 & 9.36 & 0.198 \\
\hline Mx1-NA (mm) & 5.15 & 3.89 & 6.15 & 3.20 & 0.344 \\
\hline Mx1Tip-VRP (mm) & 66.43 & 7.72 & 67.20 & 8.75 & 0.756 \\
\hline Mx6-PTV (mm) & 19.89 & 3.95 & 22.16 & 5.30 & 0.112 \\
\hline \multicolumn{6}{|c|}{ Mandibular dentoalveolar component } \\
\hline Md1-MP (mm) & 40.66 & 4.07 & 38.95 & 4.03 & 0.161 \\
\hline Md6-MP (mm) & 31.54 & 3.21 & 30.45 & 3.75 & 0.298 \\
\hline Md1-Apo (mm) & 4.06 & 2.06 & 4.89 & 3.06 & 0.297 \\
\hline \multicolumn{6}{|l|}{ Dental relationships } \\
\hline Overbite $(\mathrm{mm})$ & -3.28 & 1.85 & -3.15 & 1.82 & 0.812 \\
\hline Overjet $(\mathrm{mm})$ & 6.48 & 4.40 & -1.17 & 2.04 & $0.000^{*}$ \\
\hline Interincisal angle $\left(^{\circ}\right)$ & 119.01 & 9.58 & 129.08 & 12.11 & $0.003^{*}$ \\
\hline
\end{tabular}

${ }^{*}$ Statistically significant at $P<0.05$ 
Table IV. Treatment changes comparison between Group 1 (Class II) and Group 2 (Class III) from T2 to T1 (t test).

\begin{tabular}{|c|c|c|c|c|c|}
\hline \multirow[t]{2}{*}{ Variables } & \multicolumn{2}{|c|}{$\begin{array}{c}\text { Group } 1 \text { (Class II) } \\
(\mathrm{N}=21)\end{array}$} & \multicolumn{2}{|c|}{$\begin{array}{c}\text { Group } 2 \text { (Class III) } \\
(\mathrm{N}=25)\end{array}$} & \multirow[t]{2}{*}{$\mathbf{P}$} \\
\hline & Mean & s.d & Mean & s.d & \\
\hline \multicolumn{6}{|l|}{ Maxillary component } \\
\hline SNA $\left({ }^{\circ}\right)$ & 2.52 & 4.44 & 5.17 & 4.27 & $0.045^{*}$ \\
\hline $\mathrm{CoA}(\mathrm{mm})$ & 3.70 & 5.06 & 1.70 & 7.42 & 0.300 \\
\hline A-Nperp (mm) & 1.51 & 3.47 & 4.15 & 3.49 & $0.013^{*}$ \\
\hline \multicolumn{6}{|c|}{ Mandibular component } \\
\hline SNB $\left(^{\circ}\right)$ & 3.70 & 4.25 & 2.44 & 3.88 & 0.300 \\
\hline $\operatorname{IMPA}\left({ }^{\circ}\right)$ & 1.20 & 8.84 & -0.14 & 6.27 & 0.550 \\
\hline Pog-NB (mm) & 2.01 & 2.72 & 1.89 & 2.52 & 0.874 \\
\hline GoGn.OP( $\left(^{\circ}\right)$ & -5.11 & 6.58 & -1.58 & 3.98 & $0.030^{*}$ \\
\hline \multicolumn{6}{|c|}{ Maxillomandibular relationship } \\
\hline ANB $\left(^{\circ}\right)$ & -1.17 & 4.12 & 2.69 & 3.36 & $0.001^{*}$ \\
\hline Wits appraisal (mm) & -5.17 & 5.34 & 3.65 & 4.72 & $0.000^{*}$ \\
\hline \multicolumn{6}{|l|}{ Growth pattern } \\
\hline $\mathrm{FMA}\left({ }^{\circ}\right)$ & -3.07 & 7.26 & -4.22 & 5.40 & 0.541 \\
\hline SN.GoGn( $\left.{ }^{\circ}\right)$ & -3.82 & 7.67 & -4.80 & 5.87 & 0.624 \\
\hline SN.PP( $\left(^{\circ}\right)$ & -0.13 & 4.76 & -2.14 & 4.22 & 0.136 \\
\hline $\mathrm{SN} . \mathrm{OP}\left({ }^{\circ}\right)$ & 1.26 & 5.83 & -3.21 & 6.01 & $0.014^{*}$ \\
\hline \multicolumn{6}{|c|}{ Maxillary dentoalveolar component } \\
\hline $\mathrm{M} \times 1 . \mathrm{SN}\left({ }^{\circ}\right)$ & -4.98 & 6.14 & -0.65 & 6.53 & $0.026^{*}$ \\
\hline Mx1-NA (mm) & -1.75 & 4.11 & -1.50 & 2.78 & 0.809 \\
\hline Mx1Tip-VRP (mm) & 2.92 & 4.99 & 3.68 & 6.63 & 0.667 \\
\hline Mx6-PTV (mm) & 2.74 & 4.35 & 4.23 & 5.60 & 0.325 \\
\hline \multicolumn{6}{|c|}{ Mandibular dentoalveolar component } \\
\hline Md1-MP (mm) & 1.74 & 3.85 & -0.31 & 3.24 & 0.054 \\
\hline Md6-MP (mm) & 1.34 & 3.84 & -1.04 & 3.43 & $0.031^{*}$ \\
\hline Md1-Apo (mm) & 0.25 & 2.83 & -2.80 & 3.06 & $0.001^{*}$ \\
\hline \multicolumn{6}{|l|}{ Dental relationships } \\
\hline Overbite (mm) & 4.20 & 1.86 & 4.23 & 1.92 & 0.968 \\
\hline Overjet $(\mathrm{mm})$ & -3.94 & 4.73 & 3.66 & 2.22 & $0.000^{*}$ \\
\hline Interincisal angle $\left(^{\circ}\right)$ & 7.61 & 8.31 & 5.59 & 10.25 & 0.471 \\
\hline
\end{tabular}

*Statistically significant at $P<0.05$ 
Table V. Group 1 (Class II) to Group 2 (Class III) comparison regarding cephalometric variables at $\mathrm{T} 2$ (t test).

\begin{tabular}{|c|c|c|c|c|c|}
\hline \multirow{2}{*}{ Variables } & \multicolumn{2}{|c|}{$\begin{array}{c}\text { Group } 1 \text { (Class II) } \\
(\mathrm{N}=21)\end{array}$} & \multicolumn{2}{|c|}{$\begin{array}{c}\text { Group } 2 \text { (Class III) } \\
(\mathrm{N}=25)\end{array}$} & \multirow{2}{*}{$\mathbf{P}$} \\
\hline & Mean & s.d & Mean & s.d & \\
\hline \multicolumn{6}{|l|}{ Maxillary component } \\
\hline SNA $\left({ }^{\circ}\right)$ & 83.03 & 5.27 & 85.13 & 4.22 & 0.141 \\
\hline CoA (mm) & 81.74 & 6.12 & 80.78 & 7.17 & 0.630 \\
\hline A-Nperp (mm) & 3.27 & 3.60 & 4.12 & 3.57 & 0.426 \\
\hline \multicolumn{6}{|c|}{ Mandibular component } \\
\hline SNB $\left(^{\circ}\right)$ & 77.59 & 4.68 & 83.49 & 4.57 & $0.000^{*}$ \\
\hline IMPA $\left({ }^{\circ}\right)$ & 91.01 & 8.06 & 82.01 & 7.24 & $0.000^{*}$ \\
\hline Pog-NB (mm) & 3.09 & 2.27 & 4.04 & 2.32 & 0.170 \\
\hline GoGn.OP( $\left.{ }^{\circ}\right)$ & 20.96 & 5.87 & 21.12 & 4.97 & 0.917 \\
\hline \multicolumn{6}{|c|}{ Maxillomandibular relationship } \\
\hline ANB $\left({ }^{\circ}\right)$ & 5.45 & 3.69 & 1.63 & 2.39 & $0.000^{*}$ \\
\hline Wits appraisal (mm) & $\begin{array}{ll}-1.64 \\
\end{array}$ & 4.07 & -3.99 & 3.12 & $0.032^{*}$ \\
\hline \multicolumn{6}{|l|}{ Growth pattern } \\
\hline $\mathrm{FMA}\left({ }^{\circ}\right)$ & 30.96 & 5.77 & 25.79 & 5.68 & $0.003^{*}$ \\
\hline SN.GoGn( $\left.{ }^{\circ}\right)$ & 41.63 & 7.96 & 35.29 & 6.56 & $0.004^{*}$ \\
\hline SN.PP( $\left(^{\circ}\right)$ & 0.48 & 3.84 & -1.99 & 5.02 & 0.071 \\
\hline $\mathrm{SN} . \mathrm{OP}\left({ }^{\circ}\right)$ & 20.65 & 5.28 & 14.16 & 5.59 & $0.000^{*}$ \\
\hline \multicolumn{6}{|c|}{ Maxillary dentoalveolar component } \\
\hline Mx1.SN $\left(^{\circ}\right)$ & 100.70 & 7.14 & 108.02 & 8.05 & $0.002^{*}$ \\
\hline $\mathrm{M} \times 1-\mathrm{NA}(\mathrm{mm})$ & 3.45 & 4.10 & 4.65 & 2.64 & 0.237 \\
\hline Mx1Tip-VRP (mm) & 69.31 & 6.17 & 70.88 & 8.19 & 0.475 \\
\hline Mx6-PTV (mm) & 22.68 & 4.25 & 26.40 & 4.64 & $0.007^{*}$ \\
\hline \multicolumn{6}{|c|}{ Mandibular dentoalveolar component } \\
\hline Md1-MP (mm) & 42.40 & 4.21 & 38.64 & 4.71 & $0.006^{*}$ \\
\hline Md6-MP (mm) & 32.85 & 4.80 & 29.40 & 4.36 & $0.014^{*}$ \\
\hline Md1-Apo (mm) & 4.32 & 2.20 & 2.08 & 2.01 & $0.000^{*}$ \\
\hline \multicolumn{6}{|l|}{ Dental relationships } \\
\hline Overbite $(\mathrm{mm})$ & 0.84 & 0.56 & 1.07 & 0.66 & 0.212 \\
\hline Overjet $(\mathrm{mm})$ & 2.50 & 0.79 & 2.48 & 0.77 & 0.958 \\
\hline Interincisal angle $\left(^{\circ}\right)$ & 126.65 & 9.29 & 134.67 & 7.27 & $0.002^{*}$ \\
\hline
\end{tabular}


Table VI. Posttreatment changes comparison between Group 1 (Class II) to Group 2 (Class III) from T3 to T2 (t test).

\begin{tabular}{|c|c|c|c|c|c|}
\hline \multirow{2}{*}{ Variables } & \multicolumn{2}{|c|}{$\begin{array}{c}\text { Group } 1 \text { (Class II) } \\
(\mathrm{N}=21)\end{array}$} & \multicolumn{2}{|c|}{$\begin{array}{c}\text { Group } 2 \text { (Class III) } \\
(\mathrm{N}=25)\end{array}$} & \multirow{2}{*}{$\mathbf{P}$} \\
\hline & Mean & s.d & Mean & s.d & \\
\hline \multicolumn{6}{|l|}{ Maxillary component } \\
\hline SNA $\left({ }^{\circ}\right)$ & 0.65 & 4.65 & -0.68 & 3.45 & 0.270 \\
\hline CoA (mm) & 1.74 & 5.58 & 2.83 & 7.78 & 0.595 \\
\hline A-Nperp (mm) & -0.11 & 4.84 & -0.45 & 2.55 & 0.758 \\
\hline \multicolumn{6}{|c|}{ Mandibular component } \\
\hline SNB $\left(^{\circ}\right)$ & 0.46 & 4.64 & -0.79 & 3.80 & 0.316 \\
\hline $\operatorname{IMPA}\left({ }^{\circ}\right)$ & -0.48 & 5.96 & 1.13 & 6.69 & 0.396 \\
\hline Pog-NB (mm) & 0.34 & 2.34 & -0.25 & 1.84 & 0.337 \\
\hline GoGn.OP( $\left(^{\circ}\right)$ & 0.96 & 5.08 & 0.44 & 4.45 & 0.709 \\
\hline \multicolumn{6}{|c|}{ Maxillomandibular relationship } \\
\hline ANB $\left({ }^{\circ}\right)$ & 0.17 & 2.81 & 0.11 & 3.33 & 0.955 \\
\hline Wits appraisal (mm) & 0.96 & 3.97 & 0.33 & 4.50 & 0.617 \\
\hline \multicolumn{6}{|l|}{ Growth pattern } \\
\hline $\mathrm{FMA}\left({ }^{\circ}\right)$ & 0.30 & 5.28 & 0.73 & 6.05 & 0.802 \\
\hline SN.GoGn $\left({ }^{\circ}\right)$ & -0.68 & 5.83 & 0.85 & 6.35 & 0.401 \\
\hline SN.PP( $\left.{ }^{\circ}\right)$ & -1.45 & 4.28 & 0.67 & 3.37 & 0.065 \\
\hline $\mathrm{SN} . \mathrm{OP}\left(^{\circ}\right)$ & -1.63 & 4.61 & 0.41 & 3.03 & 0.076 \\
\hline \multicolumn{6}{|c|}{ Maxillary dentoalveolar component } \\
\hline Mx1.SN $\left(^{\circ}\right)$ & 1.66 & 7.67 & -0.21 & 6.23 & 0.365 \\
\hline Mx1-NA (mm) & -0.42 & 2.85 & -0.22 & 3.39 & 0.835 \\
\hline Mx1Tip-VRP (mm) & 1.96 & 6.55 & 0.92 & 7.23 & 0.612 \\
\hline Mx6-PTV (mm) & 0.87 & 4.42 & 0.52 & 4.44 & 0.789 \\
\hline \multicolumn{6}{|c|}{ Mandibular dentoalveolar component } \\
\hline Md1-MP (mm) & 0.49 & 3.62 & 0.77 & 3.89 & 0.802 \\
\hline Md6-MP (mm) & 0.64 & 2.12 & 0.66 & 3.12 & 0.979 \\
\hline Md1-Apo (mm) & -0.35 & 2.13 & 0.15 & 1.75 & 0.383 \\
\hline \multicolumn{6}{|l|}{ Dental relationships } \\
\hline Overbite (mm) & -0.75 & 1.28 & -0.20 & 1.81 & 0.247 \\
\hline Overjet $(\mathrm{mm})$ & 0.01 & 2.04 & -0.08 & 1.80 & 0.869 \\
\hline Interincisal angle $\left(^{\circ}\right)$ & -0.48 & 7.47 & -1.77 & 7.65 & 0.569 \\
\hline
\end{tabular}


Table VII. Group 1 (Class II) to Group 2 (Class III) comparison of cephalometric variables at T3 (t test) and percentages of patients presenting with clinically significant open bite relapse between groups (chi-square test).

\begin{tabular}{|c|c|c|c|c|c|}
\hline \multirow[t]{2}{*}{ Variables } & \multicolumn{2}{|c|}{$\begin{array}{c}\text { Group } 1 \text { (Class II) } \\
(\mathrm{N}=21)\end{array}$} & \multicolumn{2}{|c|}{$\begin{array}{c}\text { Group } 2 \text { (Class III) } \\
(\mathrm{N}=25)\end{array}$} & \multirow[t]{2}{*}{$\mathbf{P}$} \\
\hline & Mean & s.d & Mean & s.d & \\
\hline \multicolumn{6}{|l|}{ Maxillary component } \\
\hline SNA $\left({ }^{\circ}\right)$ & 83.68 & 5.13 & 84.45 & 4.92 & 0.606 \\
\hline CoA (mm) & 83.54 & 7.22 & 83.61 & 6.69 & 0.971 \\
\hline A-Nperp (mm) & 3.19 & 3.40 & 3.66 & 3.78 & 0.660 \\
\hline \multicolumn{6}{|l|}{ Mandibular component } \\
\hline SNB $\left(^{\circ}\right)$ & 77.94 & 6.43 & 82.70 & 4.71 & $0.006^{*}$ \\
\hline IMPA $\left({ }^{\circ}\right)$ & 90.57 & 5.68 & 83.14 & 8.61 & $0.001^{*}$ \\
\hline Pog-NB (mm) & 3.42 & 2.25 & 3.78 & 2.42 & 0.607 \\
\hline GoGn.OP( $\left(^{\circ}\right)$ & 22.11 & 5.15 & 21.56 & 5.54 & 0.730 \\
\hline \multicolumn{6}{|l|}{ Maxillomandibular relationship } \\
\hline ANB $\left(^{\circ}\right)$ & 5.71 & 3.63 & 1.75 & 3.51 & $0.000^{*}$ \\
\hline Wits appraisal $(\mathrm{mm})$ & -0.46 & 4.61 & -3.66 & 3.62 & $0.011^{*}$ \\
\hline \multicolumn{6}{|l|}{ Growth pattern } \\
\hline $\mathrm{FMA}\left({ }^{\circ}\right)$ & 31.34 & 7.66 & 26.53 & 5.78 & $0.019^{*}$ \\
\hline SN.GoGn $\left({ }^{\circ}\right)$ & 41.08 & 9.91 & 36.15 & 5.50 & $0.038^{*}$ \\
\hline SN.PP( $\left.{ }^{\circ}\right)$ & -0.82 & 5.01 & -1.31 & 3.60 & 0.703 \\
\hline SN.OP( $\left(^{\circ}\right)$ & 18.96 & 7.04 & 14.58 & 4.87 & $0.016^{*}$ \\
\hline \multicolumn{6}{|l|}{ Maxillary dentoalveolar component } \\
\hline Mx1.SN $\left({ }^{\circ}\right)$ & 102.21 & 10.14 & 107.81 & 8.26 & $0.045^{*}$ \\
\hline $\mathrm{M} \times 1-\mathrm{NA}(\mathrm{mm})$ & 2.98 & 2.60 & 4.42 & 2.95 & 0.088 \\
\hline Mx1Tip-VRP (mm) & 71.32 & 9.82 & 71.80 & 7.53 & 0.852 \\
\hline Mx6-PTV $(\mathrm{mm})$ & 23.51 & 4.14 & 26.92 & 5.40 & $0.022^{*}$ \\
\hline \multicolumn{6}{|l|}{ Mandibular dentoalveolar component } \\
\hline Md1-MP (mm) & 42.90 & 4.18 & 39.41 & 4.75 & $0.012^{*}$ \\
\hline Md6-MP (mm) & 33.53 & 4.84 & 30.07 & 4.12 & $0.011^{*}$ \\
\hline Md1-Apo (mm) & 3.96 & 2.04 & 2.24 & 2.32 & $0.011^{*}$ \\
\hline \multicolumn{6}{|l|}{ Dental relationships } \\
\hline Overbite $(\mathrm{mm})$ & 0.17 & 1.06 & 0.87 & 1.77 & 0.118 \\
\hline Overjet (mm) & 2.54 & 1.74 & 2.40 & 1.49 & 0.765 \\
\hline Interincisal angle $\left({ }^{\circ}\right)$ & 126.15 & 8.02 & 132.90 & 12.93 & $0.043^{*}$ \\
\hline $\begin{array}{c}\text { Clinically significant stability/relapse } \\
\text { Percentage of patients } \\
(\%) \\
\text { Number of patients }\end{array}$ & $\begin{array}{l}\text { Stability } \\
\begin{array}{c}57.14 \% \\
\mathrm{n}=12\end{array}\end{array}$ & $\begin{array}{c}\text { Relapse } \\
\begin{array}{c}42.86 \% \\
n=9\end{array}\end{array}$ & $\begin{array}{l}\text { Stability } \\
\begin{array}{l}88.00 \% \\
\mathrm{n}=22\end{array}\end{array}$ & $\begin{array}{l}\text { Relapse } \\
\begin{array}{l}12 \% \\
\mathrm{n}=3\end{array}\end{array}$ & $0.017 ¥$ \\
\hline
\end{tabular}

*Statistically significant at $P<0.05$

$¥$ chi-square 
Table VIII. Intergroup comparability regarding the type of fixation (chi-square).

\begin{tabular}{|c|c|c|c|}
\hline $\begin{array}{c}\text { Type of Fixation } \\
\text { Group }\end{array}$ & Wire (WF) & Rigid (RIF) & Total \\
\hline G1(Class II) & 8 & 13 & 21 \\
\hline G2(Class III) & 6 & 19 & 25 \\
\hline Total & 14 & 32 & 46 \\
\hline
\end{tabular}



4 Discussion 



\section{DISCUSSION}

Long-term stability of surgical-orthodontic open bite treatment is a controversial issue which has brought interest not only to orthodontists but also to oral surgeons over the last decades. However, the inner difficulties in obtaining a proper prospective study design, such as randomized clinical trials with standardized orthodontic and surgical treatment, adequate sample size, isolating the possible influencing factors, with reliable posttreatment follow-up (at least 5 years), ended up limiting the available evidence.(HUANG, 2002; GREENLEE et al., 2011; FONTES et al., 2012) Retrospective studies, despite its limitations, can also bring important information to clinicians in order to answer clinical focal questions.

Firstly, is important to differentiate the results considering 4 correlated situations one can find: 1- Influence of orthodontic pretreatment (dentoalveolar) on the final overbite; 2- Skeletal stability/instability of the surgical procedure on the vertical plane; 3- Overbite dentoalveolar compensation maintaining the overbite; 4Posttreatment factors (tongue thrust, condiles resorption). The interaction between these 4 processes lead to the long-term stability/relapse of open bite and in our study, the main objective is to assess the final result of overbite measurement. Some authors concluded that no difference was found on the vertical plane between 1piece Le Fort I and 3-piece Le Fort I osteotomies (BAILEY et al., 1994a; HOPPENREIJS et al., 1997; PEREZ; SAMESHIMA; SINCLAIR, 1997). While others, considering the final overbite result regardless of what led to the overbite stability/instability, found that 1-piece was more stable(FISCHER; VON KONOW; BRATTSTROM, 2000; KRETSCHMER et al., 2010; ISMAIL; LEUNG, 2017) and some others considered 1-piece less stable than segmental ones such as 3piece(TURVEY et al., 1988; ARPORNMAEKLONG; HEGGIE; SHAND, 2003). In this study, the percentages of stable subjects showed that, despite the higher stability percentage in Group $2(83.33 \%)$ than in Group 1(65.52\%), results were not statistically significant (Table VII). In Group 1 (1-piece), majority of subjects (19 out of 29 patients) remained with an overbite greater than $0 \mathrm{~mm}$. in Group 2 (4-piece) 20 out of 24 patients remained with a positive overbite after the long-term observation 
period. Subgroup 1 (WF) contributed with 8 subjects presenting with clinical overbite relapses and a statistically significant negative overbite mean of $-0.18 \mathrm{~mm}$. In Subgroup 2 (RIF), only 2, out of 10 subjects, presented a negative overbite at T3 (follow-up). In other words, fixation type seemed to influence overbite stability in the long-term more than surgery type.

Regarding the anteroposterior comparison on the long-term stability between Class II and Class III open bite subjects, the mandibular retrusion in Class II group and the maxillary retrusion with well positioned mandible in Class III group showed that anteroposterior discrepancy, in fact, the clock-wise mandible rotation direction is one of the in the high-angle patients (JANSON et al., 2016). However, Class II group presented with a stronger vertical growth tendency, but due to the fact that patients were not in active growing, this difference must have not influenced the results. The relapse of mandibular plane correction is, usually, associated to overbite relapse in surgical-orthodontic treatment of Class II and III open bite patients (REYNEKE et al., 2007; KOR; YANG; HWANG, 2014), and skeletal changes tends to be counteracted by dentoalveolar alterations (SOLOW, 1980). In the end of the posttreatment phase, despite, the final overbite means has not showed difference among groups, Class II group tended to an open bite (overbite $<0 \mathrm{~mm}$ ), whereas, Class III mean was $0.87 \mathrm{~mm}$. As the main objective of this study, overbite stability at the end of posttreatment phase showed that 9 out of 21 patients in Class II group presented a clinical relapse. Class III group presented only 3 out of 25 subjects with an overbite smaller than Omm, corroborating several other studies,(FISCHER; VON KONOW; BRATTSTROM, 2000; SWINNEN et al., 2001; IANNETTI et al., 2007; ESPELAND et al., 2008; JAKOBSONE et al., 2011). So, with stronger evidence, because most of those studies were based on short-term follow-up and small sample size, our results confirm that Class II surgical-orthodontic treatment of open bite patients is less stable than Class III treatment. 
5 CONCLUSIONS 



\section{CONCLUSIONS}

The first null hypothesis was accepted because:

- There was no significant intergroup difference regarding the percentage of clinically stable patients;

- The type of fixation seems to influence the long-term stability of open bite surgical-orthodontic correction more than maxillary segmentation.

The second null hypothesis was rejected because:

- Clinical stability of Class II malocclusion open bite surgical-orthodontic treatment was significantly smaller than in Class III malocclusions. 



\section{REFERENCES}





\section{REFERENCES}

Arpornmaeklong $\mathrm{P}$, Heggie AA, Shand JM. A comparison of the stability of singlepiece and segmental Le Fort I maxillary advancements. J Craniofac Surg. 2003 Jan;14(1):3-9.

Bailey L, Phillips C, Proffit W, Turvey T. Stability following superior repositioning of the maxilla by Le Fort I osteotomy: five-year follow-up. Int J Adult Orthod Orthognath Surg. 1994a 9(3):163-73.

Bailey LJ, Phillips C, Proffit WR, Turvey TA. Stability following superior repositioning of the maxilla by Le Fort I osteotomy: five-year follow-up. Int J Adult Orthodon Orthognath Surg. 1994b 9(3):163-73.

Epker BN, Fish LC. Surgical-orthodontic correction of open-bite deformity. Am J Orthod. 1977 71(3):278-99.

Espeland L, Dowling PA, Mobarak KA, Stenvik A. Three-year stability of open-bite correction by 1-piece maxillary osteotomy. Am J Orthod Dentofacial Orthop. 2008 Jul;134(1):60-6.

Fischer K, von Konow L, Brattstrom V. Open bite: stability after bimaxillary surgery-2-year treatment outcomes in 58 patients. Eur J Orthod. 2000 Dec;22(6):711-8.

Fischer K, von Konow L, Brattström V. Open-bite: stability after bimaxillary surgery-2year treatment outcomes in 58 patients. Eur J Orthod. 2000 22(6):711-8.

Fontes AM, Joondeph DR, Bloomquist DS, Greenlee GM, Wallen TR, Huang GJ. Long-term stability of anterior open-bite closure with bilateral sagittal split osteotomy. Am J Orthod Dentofacial Orthop. 2012 Dec;142(6):792-800.

Greenlee GM, Huang GJ, Chen SS, Chen J, Koepsell T, Hujoel P. Stability of treatment for anterior open-bite malocclusion: a meta-analysis. Am J Orthod Dentofacial Orthop. 2011 Feb;139(2):154-69.

Hoppenreijs TJM, Freihofer HPM, Stoelinga PJW, Tuinzig DB, van't Hof MA, Nottet SJAM. Skeletal and dentoalveolar stability of Le Fort I intrusion osteotomies and bimaxillary osteotomies in anterior open bite deformities. A retrospective three-centre study. Int J Oral Maxillofac Surg. 1997 26(161-75. 
Huang GJ, editor Long-term stability of anterior open-bitetherapy: A review. Semin Orthod; 2002: Elsevier.

lannetti G, Fadda MT, Marianetti TM, Terenzi V, Cassoni A. Long-term skeletal stability after surgical correction in Class III open-bite patients: a retrospective study on 40 patients treated with mono- or bimaxillary surgery. The Journal of craniofacial surgery. 2007 Mar;18(2):350-4.

Ismail I, Leung Y. Anterior open bite correction by Le Fort I osteotomies with or without anterior segmentation: which is more stable? Int J Oral Maxillofac Surg. 2017 46(159.

Jakobsone G, Stenvik A, Sandvik L, Espeland L. Three-year follow-up of bimaxillary surgery to correct skeletal Class III malocclusion: stability and risk factors for relapse. Am J Orthod Dentofacial Orthop. 2011 Jan;139(1):80-9.

Janson G, Laranjeira V, Rizzo M, Garib D. Posterior tooth angulations in patients with anterior open bite and normal occlusion. Am J Orthod Dentofacial Orthop. 2016 150(1):71-7.

Kahnberg KE. Transverse expansion of the maxilla using a multisegmentation technique. Scand J Plast Reconstr Surg Hand Surg. 2007 41(3):103-8.

Kahnberg KE, Vannas-Lofqvist L, Zellin G. Complications associated with segmentation of the maxilla: a retrospective radiographic follow up of 82 patients. Int J Oral Maxillofac Surg. 2005 Dec;34(8):840-5.

Kor HS, Yang HJ, Hwang SJ. Relapse of skeletal class III with anterior open bite after bimaxillary orthognathic surgery depending on maxillary posterior impaction and mandibular counterclockwise rotation. J Craniomaxillofac Surg. 2014 Jul;42(5):e2308.

Kretschmer WB, Baciut G, Baciut M, Zoder W, Wangerin K. Stability of Le Fort I osteotomy in bimaxillary osteotomies: single-piece versus 3-piece maxilla. J Oral Maxillofac Surg. 2010 Feb;68(2):372-80.

Maia FA, Janson G, Barros SE, Maia NG, Chiqueto K, Nakamura AY. Long-term stability of surgical-orthodontic open-bite correction. Am J Orthod Dentofacial Orthop. 2010 Sep;138(3):254 e1- e10; discussion -6.

Medeiros RB, de Araújo LFC, Mucha JN, Motta AT. Stability of open-bite treatment in adult patients: A systematic review. World J Orthod. 2012 1(3):e97-e101. 
Obwegeser $\mathrm{H}$. [The one time forward movement of the maxilla and backward movement of the mandible for the correction of extreme prognathism]. SSO Schweiz Monatsschr Zahnheilkd. 1970 May;80(5):547-56.

Perez MMC, Sameshima GT, Sinclair PM. The long-term stability of LeFort I maxillary downgrafts with rigid fixation to correct vertical maxillary deficiency. Am J Orthod Dentofacial Orthop. 1997 112(1):104-8.

Posnick JC, Adachie A, Choi E. Segmental Maxillary Osteotomies in Conjunction With Bimaxillary Orthognathic Surgery: Indications-Safety-Outcome. J Oral Maxillofac Surg. 2016 74(7):1422-40.

Proffit WR, White J. Combined surgical-orthodontic treatment: How did it evolve and what are the best practices now? Am J Orthod Dentofacial Orthop. 2015 147(S):20515.

Reyneke JP. Vertical variation in skeletal open bite: a classification for surgical planning. J Dent Ass S Africa. 1988 43(465-72.

Reyneke JP, Bryant RS, Suuronen R, Becker PJ. Postoperative skeletal stability following clockwise and counter-clockwise rotation of the maxillomandibular complex compared to conventional orthognathic treatment. Br J Oral Maxillofac Surg. 2007 Jan;45(1):56-64.

Reyneke JP, Ferretti C. Anterior open bite correction by Le Fort I or bilateral sagittal split osteotomy. Oral Maxillofacial Surg Clin N Am. 2007 19(3):321-38.

Solano-Hernandez B, Antonarakis GS, Scolozzi P, Kiliaridis S. Combined orthodontic and orthognathic surgical treatment for the correction of skeletal anterior open-bite malocclusion: a systematic review on vertical stability. J Oral Maxillofac Surg. 2013 Jan;71(1):98-109.

Solow B. The dentoalveolar compensatory mechanism: background and clinical implications. J Orthod. 1980 July 1, 1980;7(3):145-61.

Subtelny JD, Sakuda M. Open-bite: diagnosis and treatment. Am J Orthod. 1964 50(5):337-58.

Swinnen K, Politis C, Willems G, De Bruyne I, Fieuws S, Heidbuchel K, et al. Skeletal and dento-alveolar stability after surgical-orthodontic treatment of anterior open bite: a retrospective study. Eur J Orthod. 2001 23(5):547-57. 
Turvey TA, Philips C, Zaytoun HS, Proffit WR. Simultaneous superior repositioning of the maxilla and mandibular advancement. Am J Orthod. 1988 94(372-83. 
APPENDIXES 



\section{APPENDIX A - Declaration of exclusive use of the article 1 in thesis}

\section{DECLARATION OF EXCLUSIVE USE OF ARTICLE IN THESIS}

We hereby, declare we are aware that the article "Long-term stability of surgicalorthodontic open bite treatment: Le Fort I versus 4-piece segmental Le Fort I osteotomy" will be included in the thesis of the student Vinicius Laranjeira Barbosa da Silva and may not be used in any other scientific production of the Graduate Program at Bauru Dental School, University of São Paulo.

Vinicius Laranjeira Barbosa da Silva

Bauru, January 18, 2018.

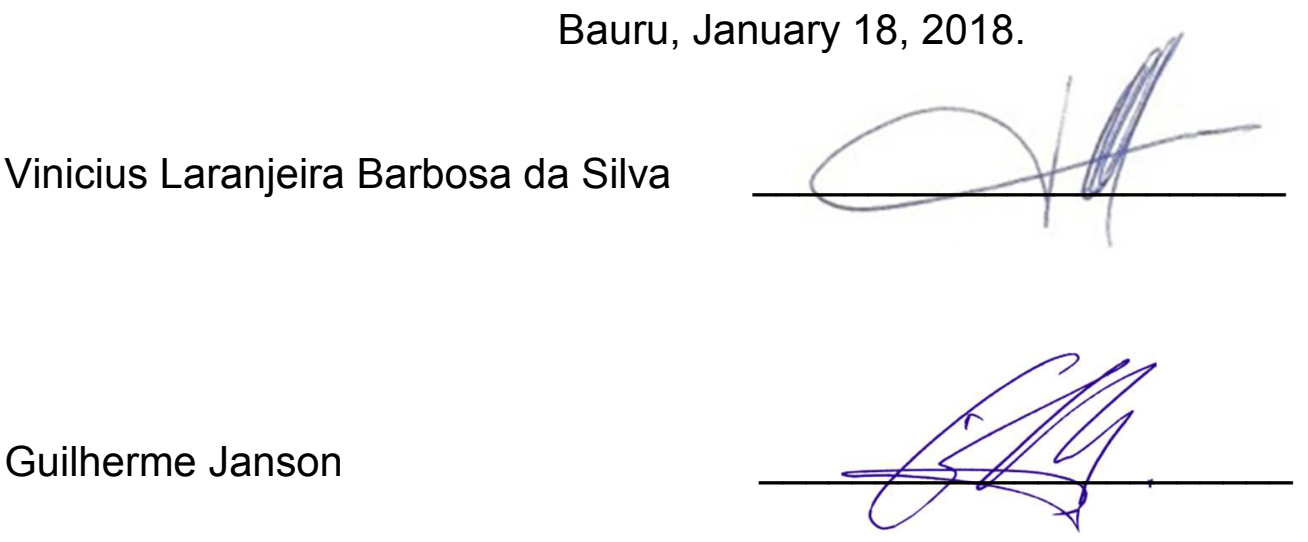


APPENDIX B - Declaration of exclusive use of the article 2 in thesis

\section{DECLARATION OF EXCLUSIVE USE OF ARTICLE IN THESIS}

We hereby, declare we are aware that the article "Long-term stability of surgicalorthodontic open bite treatment: Class II versus Class III" will be included in the thesis of the student Vinicius Laranjeira Barbosa da Silva and may not be used in any other scientific production of the Graduate Program at Bauru Dental School, University of São Paulo.

Bauru, January 18, 2018.

Vinicius Laranjeira Barbosa da Silva

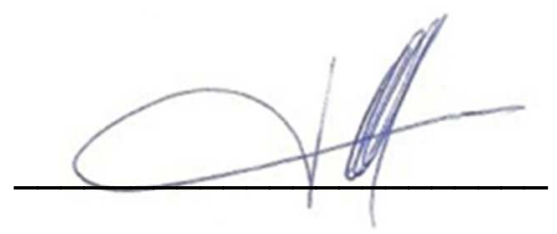

Guilherme Janson

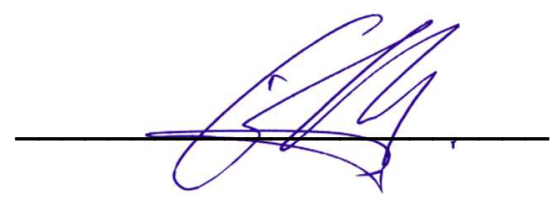


ANNEX 



\section{ANNEX A - Institutional Review Board approval, protocol number 71636716.5.0000.5417 (front page)}

\section{USP - FACULDADE DE ODONTOLOGIA DE BAURU DA USP}

\section{PARECER CONSUBSTANCIADO DO CEP}

\section{DADOS DO PROJETO DE PESOUISA}

Titulo da Pesquisa: Estabilidade em lonoo prazo do tratamento cirvirgico da mordida aberta anterior por osteo lomia tipo Le For I com e sem segmentacja maxilat

Pesquisador: Vinicus Laranjeira Barbosa da Silva

Area Temática:

Versāo: 2

CAAE: $71636716,5,0000.5417$.

Instituáçāo Proponente: Universidade de Sao Paulo

Patrocinador Principal: Financiamerio Próorio

DADOS DO PARECER

Nimero do Parecer. 2.332 .849

Apresentaçào do Proje to:

As dificuldaches de tratamento e a ba ixa esfabilikade ros casos de Mordicta Aberla Anterior, me mo em casos fratados cirurgicamente, sajo motivos de frusbraçäo para pacientes e profissionsis: $O$ avastco e surgimento de novas têtröcas cirurgicas podert, em looria, Irazor maicr estabilidade em longo prazo no tratamento dessa desafiante ma-oclusâ. A técnica de osteotomia tipo Le Fort I segmentada da maxila é uma promissora abordayom que aponta para uma maior estabilidade, no entanto, existem poucos estudos consistentes e especificos que avaliaram seus resallados em longo prazo na correcaio da mordida aber ta anterion, Portanto, o objetive desse trabaltho ê lestar a hipólese nula de que nảo hâ diferença quanto a

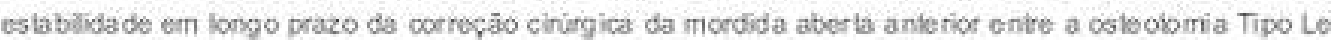
Fort I com e som segmentac; alo maxilar. A atmostra serd composta por 162 Tele rtadiografias previamontie obtidas de 54 pacierdes tra tados em consultórios privados de Bauru, Araraquara e Sajo Paulo. Seräo divididos em dois grupos: Grupo Sem Segmentaçäo (29 pacientes) e Grupo Com Segmentaçäo (25 pacientes). As alkera T2 (imediatarnen te apds a remoyajo do apare ho) e T3 (no minimo 2 ancos após a re moça do apareliho).

Objetivo da Pesquisa:

Comparar a estabilidado em longo prazo da corre çäo cinurgica da mordida aberta anterior entre as

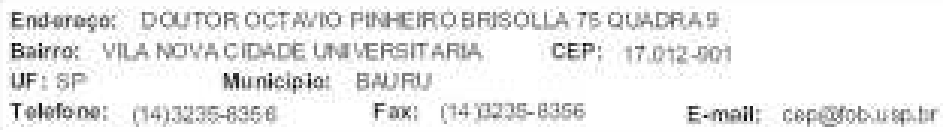




\section{ANNEX A - Institutional Review Board approval letter, protocol number 71636716.5.0000.5417 (back page)}

USP - FACULDADE DE
ODONTOLOGIA DE BAURU DA S plateforma
USP

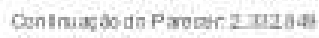

dues técricas empregadas.

Avaliaçào dos Riscos e Beneficios:

Riscos: Nấo há riscos para os sujedos da pesquisa, pois trata-se de um estudo teirospoctivo no cual as radiografias utilizadas considen nos prontudios dos mosnos. Haverd atertyijo paira manter sigilo dos nomes dos sujeritos.

Beneficios: Os beneficios impaclam diretamente sa forma de tralamerto do uma complexa má ochusảo, influonciando na decisajo dos ortodontistas e cinurgiôes quanto á claboracajo do plano do tra tamento de pacientes aduliog com mordidas aberlas severas.

Comentàrios e Consideraçōes sobre a Pesquísa:

Todas as inconsistènclas motodologicas foram esclarccidas tanto no projoto corro na. PB, porem 0 cronograma esta incorreto, pois as atividades se iniciaram antes da aprovaça do CEP,

Em relacäo ao presnchimento do PB, algumas sugostóos:

1) Deserho: substituir "54 documentaç̄es" por "54 sujpitos da pesquisa", Evitar o uso do termo "paciente":

2) Desfucho primário: Inserir os paratmetros utilizados para at nakisar estabīidade.

3) Conrigir cronograma,

Consideraçōes sobre os Termos de apresentaçào obriga tória:

Os termos estāo apropriados.

Recomendaçōes:

Pos favos, corrigit o cronograma.

Conclusōes ou Pendèncias e Lista de lnadecuaçōes:

Nenthumb

Consideraçōes Finais a critério do CEP:

Esso projeto foi consicterado APROVADO na reunijo ordinjitia do CEP de 11/10/2017, com baso nas

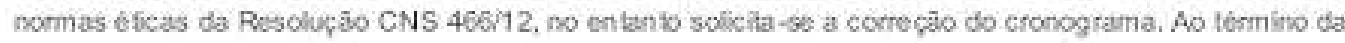
pesquisa o CEP.FOBVUSP exige a apresersiaça de relatóno final. Os relatónics parciais deveráo estar de acordo com o cron ograma e,lou parecer emifido pelo CEP. Alloraç̄os na metodologia, tîǘ, inclusão ou exclusẫo de autores, cronograma e qualsquer outras mudanças quo scjam sïnn ificativas deverảo sar previamente comunicadas a este CEP 506 risco de näo aprovacto do relatório final, Quando da apresentacäo deste, deveräo ser incluidos todos os TCLEs eiou inrmos de doaçäo assirados e nubricados, so per finenices,

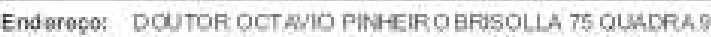

Bairro: VLA NOVACIOADE UNVEFSITAFIA CEP: 17,012-001

UF: SP Municipio; BAuRU

Telefone: (14)3235-8356 Fax: (14) $2835-8356$ E-mail: cepergobumber 


\section{ANNEX A - Institutional Review Board approval, protocol number 71636716.5.0000.5417 (front page)}

\section{USP - FACULDADE DE ODONTOLOGIA DE BAURU DA USP}

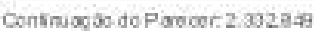

Este parecer foi elaborado baseado nos documentos abaixo rolacionados:

\begin{tabular}{|c|c|c|c|c|}
\hline Tpo Documento & Anquivo & Postalgom & Autor & Stus pajo \\
\hline $\begin{array}{l}\text { Informacōos Basices } \\
\text { do Provito }\end{array}$ & $\begin{array}{l}\text { PB_INFORAACCOES_BASICAS_DO_P } \\
\text { ROJETO } 664401 \text { pdf }\end{array}$ & $\begin{array}{l}14: 09: 2017 \\
21: 43: 47\end{array}$ & & Acceib \\
\hline Outros & QuestionarioTecniko_Revisado.pdf & $\begin{array}{c}14009 / 2017 \\
21: 05: 52\end{array}$ & $\begin{array}{l}\text { Vinicius L aranjeira } \\
\text { Barbossa da Stva }\end{array}$ & Aceito \\
\hline $\begin{array}{l}\text { TCLE / Termos do } \\
\text { Assontimento / } \\
\text { Jus lificativa do } \\
\text { Ausulncia. }\end{array}$ & JusfifcafivadeD isperisadoTCLE.pdf & $\begin{array}{l}14,09 r 2017 \\
21: 04: 44\end{array}$ & $\begin{array}{l}\text { Vinicius L aranjuira } \\
\text { Barbosa da Siva }\end{array}$ & Aceilb \\
\hline $\begin{array}{l}\text { Declaracaio de } \\
\text { Pesquisadores }\end{array}$ & OficioPesquisadorsdf & $\begin{array}{c}14 / 09 / 2017 \\
21: 04: 01\end{array}$ & $\begin{array}{l}\text { Viniclus L aranyeira } \\
\text { Barbosa da Siva }\end{array}$ & Aceito \\
\hline $\begin{array}{l}\text { Projelo De lathado f } \\
\text { Brochura. } \\
\text { Investinador }\end{array}$ & Projoto_Rievisado docx & $\begin{array}{c}14: 09 / 2017 \\
21: 03: 21\end{array}$ & $\begin{array}{l}\text { Vinicius Laranjeira } \\
\text { Barbosa da Sivar }\end{array}$ & Aceito \\
\hline $\begin{array}{l}\text { Declaracejo do } \\
\text { Pesquisadores }\end{array}$ & Termo_de_col & $\begin{array}{c}30 / 05 / 2017 \\
11: 07: 04\end{array}$ & $\begin{array}{l}\text { Vinious L aranjeira } \\
\text { Barbosa da Stiva }\end{array}$ & Accilo \\
\hline $\begin{array}{l}\text { Declaraçjo do } \\
\text { Pescuisadores }\end{array}$ & Termo_autoriza_Sao_Paulo,pdi & $\begin{array}{l}30 / 05 / 2017 \\
11: 04: 06\end{array}$ & $\begin{array}{l}\text { V/nichus L aranjeira } \\
\text { Barboso da Siva }\end{array}$ & Aceib \\
\hline $\begin{array}{l}\text { Doclaracjio do } \\
\text { Pespuisadores }\end{array}$ & Termo_autoriza_Bauru.pdf & $\begin{array}{c}30 / 05 / 2017 \\
11: 02: 50\end{array}$ & $\begin{array}{l}\text { Vincius Laranjeira } \\
\text { Barbosa da Stiva }\end{array}$ & Accilo \\
\hline $\begin{array}{l}\text { Declararajo do } \\
\text { Pesyuigadores }\end{array}$ & Termo_autonza_Anaraquara.pdf & $\begin{array}{c}30 / 05: 2017 \\
11: 02: 30\end{array}$ & $\begin{array}{l}\text { Vinicius L aranjeira } \\
\text { Barbosa da Stusa }\end{array}$ & Acceito \\
\hline $\begin{array}{l}\text { Declarapdo do } \\
\text { Pespuisadones }\end{array}$ & DeclaracacodeConfiden cialidade.pdf & $\begin{array}{c}30 / 05 / 2017 \\
10: 58: 12\end{array}$ & $\begin{array}{l}\text { Vinicius Laranjeira } \\
\text { Barbosa da Silva }\end{array}$ & Aceilis \\
\hline 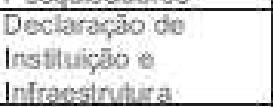 & Termo_do_Aquicscencia, pdl & $\begin{array}{l}30 / 05 / 2017 \\
10: 57: 03\end{array}$ & $\begin{array}{l}\text { Vinicus Laranjeita } \\
\text { Barbosa da Siva }\end{array}$ & Acceibs \\
\hline Folha do Rosio & Foin adeRosio Finali,poll & $\begin{array}{l}30 / 05 / 2017 \\
10: 55: 43\end{array}$ & $\begin{array}{l}\text { Vinious L aranieira } \\
\text { Barbosg da Sivgs. }\end{array}$ & Acceilo \\
\hline
\end{tabular}

Situaçăo do Parecer:

Aprovado

Necesstha Apreckaç ão da CONEP:

Nảㅇำ

BAURU, 17 de Qutubro de 2017

Assinado por:

Ana Lúcia Pompó ia Fraga de Alme ida

(Coordenador)

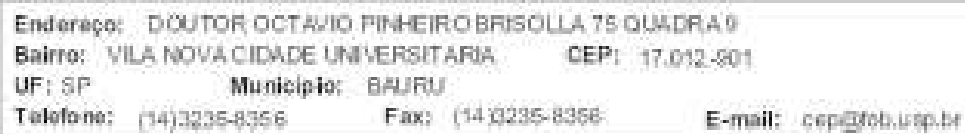




\title{
ANNEX B - Letter justifying Informed consent dismissal
}

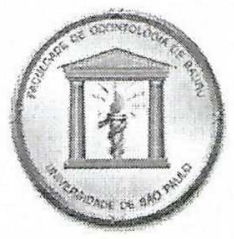

\author{
Universidade de São Paulo \\ Faculdade de Odontologia de Bauru
}

Departamento de Odontopediatria,

Ortodontia e Saúde Coletiva

\section{Justificativa de Dispensa ao TCLE}

Este documento tem por finalidade a solicitação da dispensa do Termo de Consentimento Livre e Esclarecido junto aos pacientes da amostra da pesquisa de Doutorado intitulada "Estabilidade em longo prazo do tratamento cirúrgico da mordida aberta anterior por osteotomia tipo Le Fort I com e sem segmentação maxilar", sob responsabilidade do pesquisador e aluno do Programa de Ciências Odontológicas Aplicadas da Faculdade de Odontologia de Bauru, Vinicius Laranjeira Barbosa da Silva N/USP 4790135 e de seu orientador Prof. Dr. Guilherme Janson. Para tal, seguem as justificativas abaixo:

- Trata-se de um estudo retrospectivo, o qual se utilizará de telerradiografias previamente obtidas em consultórios privados de Bauru, Araraquara e São Paulo.

- Essas radiografias já fazem parte do protocolo de diagnóstico, tratamento e proservação dos profissionais envolvidos e portanto não foram obtidas especificamente para o estudo.

- A especificidade do assunto abordado, o que não diminui sua importância clínico/científica, pelo contrário, valoriza o esforço em busca da resposta à pergunta focal do estudo, corroborado à escassez de trabalhos na literatura, ratificam a grande dificuldade em coletar essa amostra.

- Muitos pacientes se mudaram, não temos contato ou até foram à óbito. As radiografias foram obtidas com uma variabilidade grande tempo, abrangendo o período de 1988 à 2010, tornando quase impossível a obtenção do TCLE dos participantes.

- Para assegurar o anonimato dos pacientes, os consultórios privados, na pessoa de seu respectivo responsável técnico, apresentam um termo de autorização assinado para análise dos prontuários (ANEXOST, II e III).

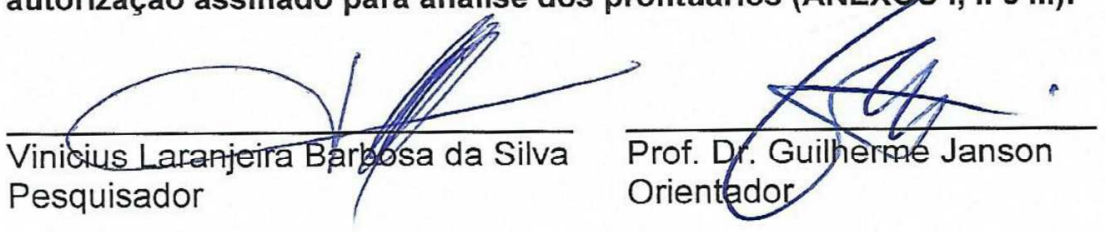

Bauru, 05 de abril de 2017.

Al. Dr. Octávio Pinheiro Brisolla, 9-75 - Bauru-SP - CEP 17012-901 - C.P. 73

e-mail: veragato@fob.usp.br - Fone/FAX (0xx14) 32358217

http://www.fob.usp.br 


\section{ANNEX C - AJO-DO manuscript submission guidelines}

$2201 / 2018$

American Joumal of Orthodontcs and Dentofaclas Orthopedce

aneral intoemation

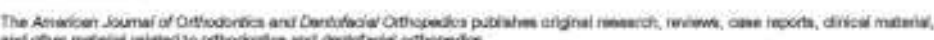

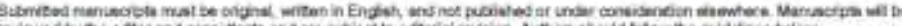

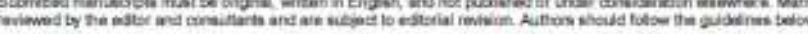

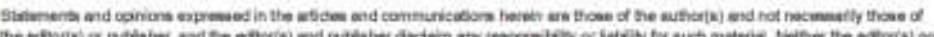

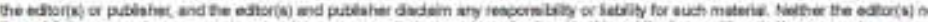

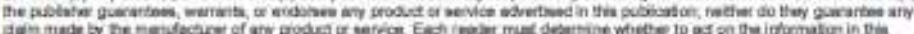

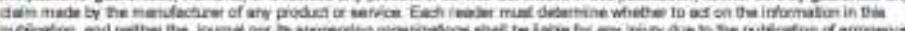

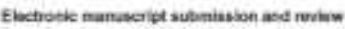

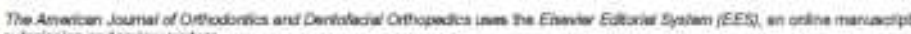

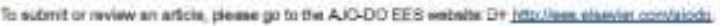

Roulc Batrmb Ealsitioned

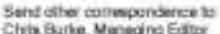

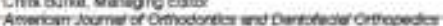

Untrensisy of Wienirum

Hsc enix 3574 es

Sinan Whestos.7400

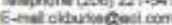

Q Beforo You Begin

Ettica in poblisting

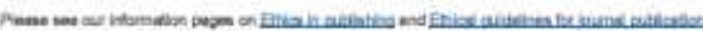

Humas and animat rights

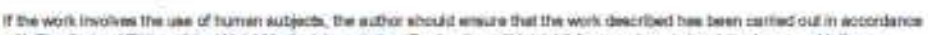

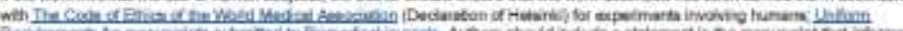

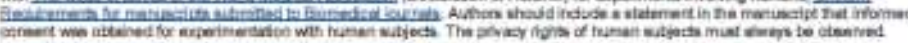

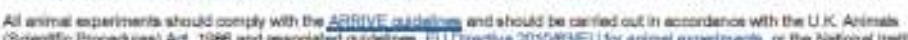

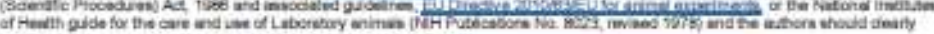

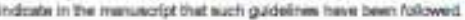

Contiat of interes

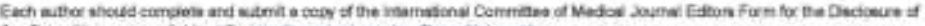

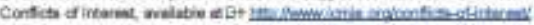

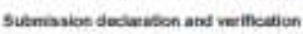

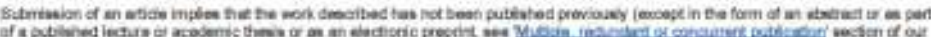

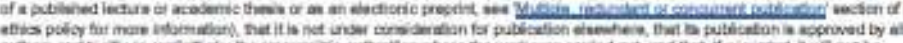

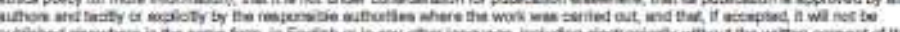

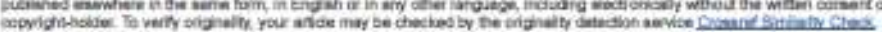

Cantibuton

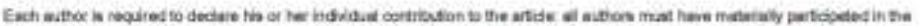

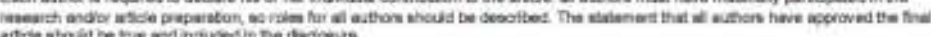
end injusedin ne dediowit:

Chenpes to authoratip

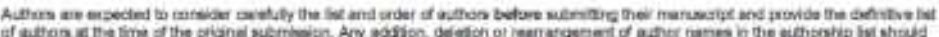

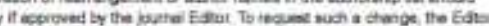
作

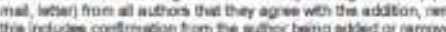

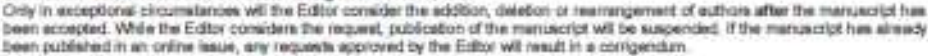

Copgright

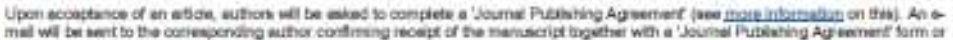

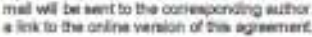

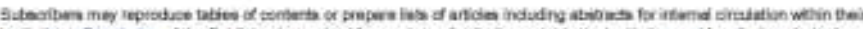

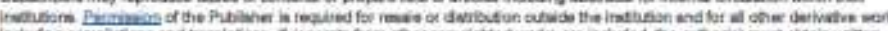

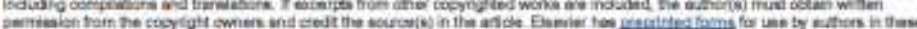




\section{ANNEX C - AJO-DO manuscript submission guidelines}

$2201 / 2018$

American Joumal of Orthodonscs and Dentofacas Orthopedce

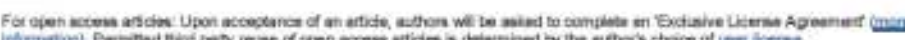

Auter nohtz

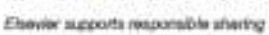

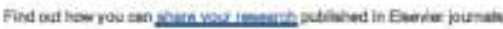

Mroke of the fursting sourcos

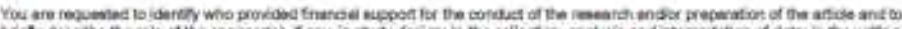

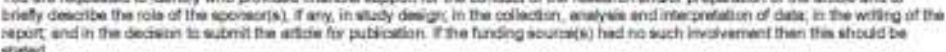

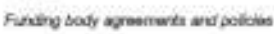

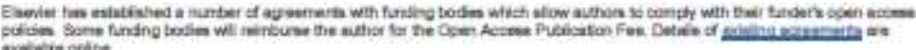

open moses

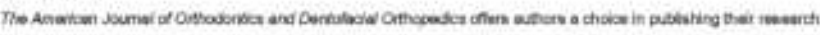

Dodecond Rocan:

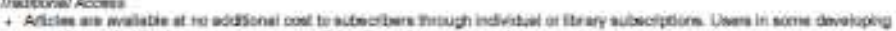

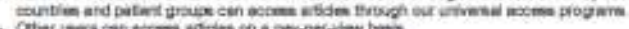

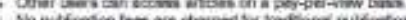

Qsen ascous

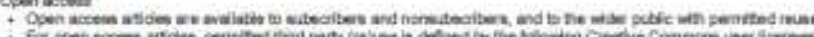

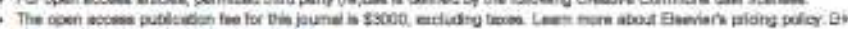

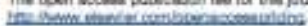

Cromen opto acras:

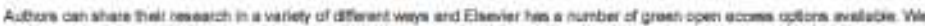

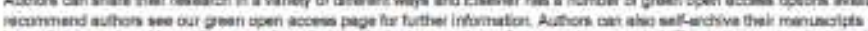

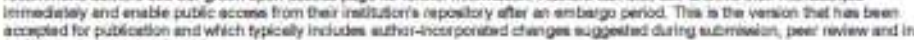

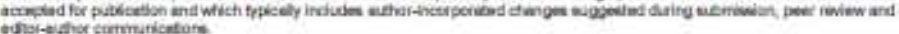

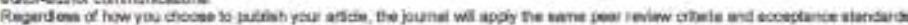

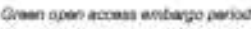

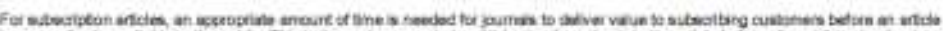

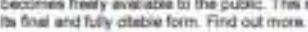

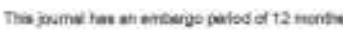

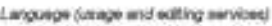

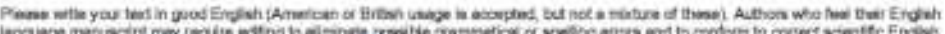
fre wal to

Informed corsent and petiet detalt

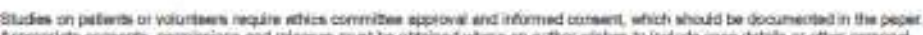

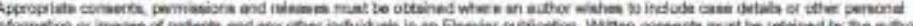

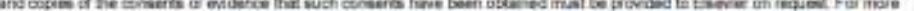

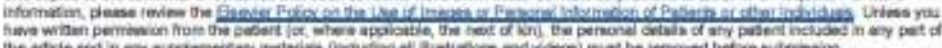

Sultaisation

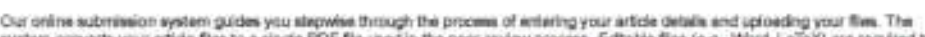

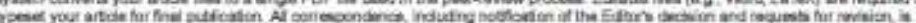
wirets ond

stinding

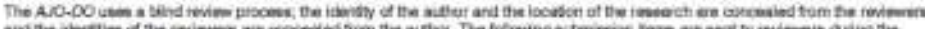

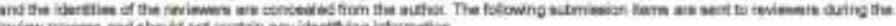

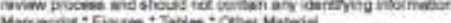

Marse

.

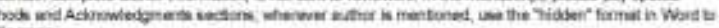

Ocidatine sor Croinal Aeticies

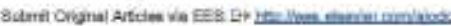

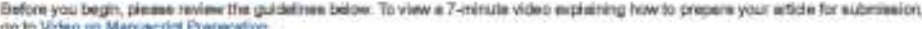

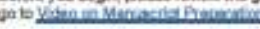




\title{
ANNEX C - AJO-DO manuscript submission guidelines
}

\author{
$22 / 01 / 2018$
}

American Joumal of Orthodontcs and Dentofacla: Orthopedce

Cluodiat be Aufsers

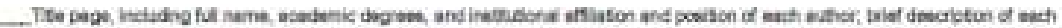

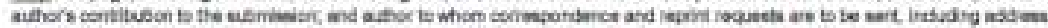

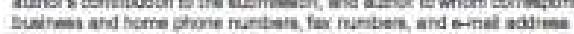

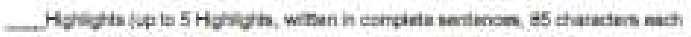

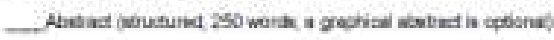

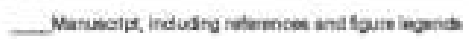

Fogra in TIF of ERS format

- Taidion

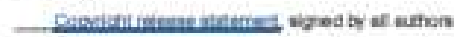

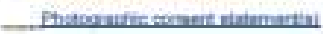

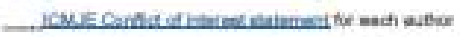

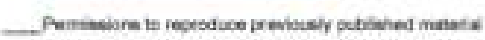

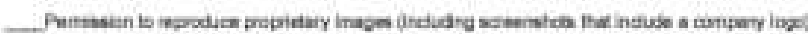

Q. Preparation

Doute-bind reakew

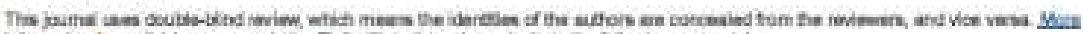

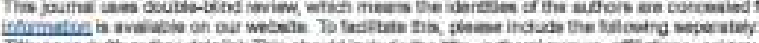

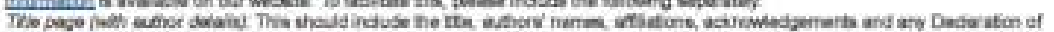

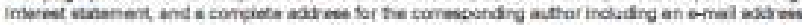

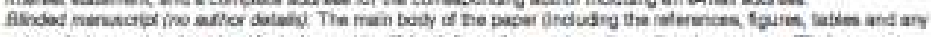

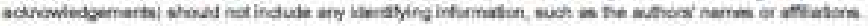

Artice structure

mbidetion

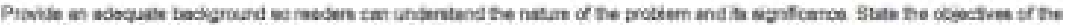

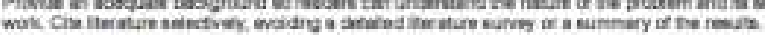

Mutonal and Mancets

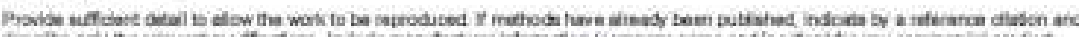

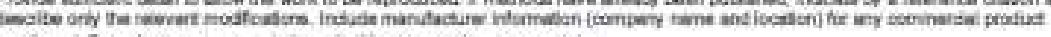

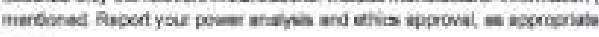

frash:

Aim its stould be dest and unean

Davasaion

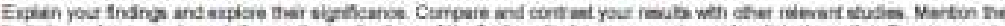

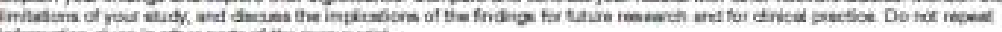
vetumition gran in other pass of lle maruactpe

Cansuetinn

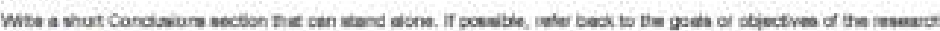

Easental utis paos information

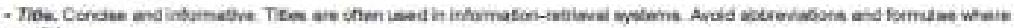
vovitis

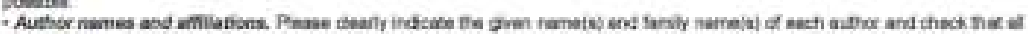

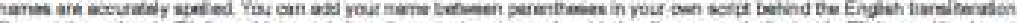

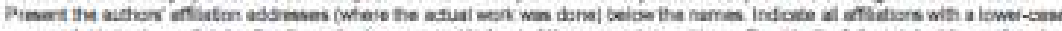

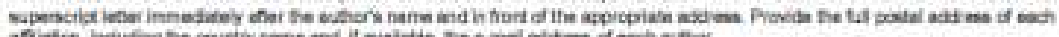

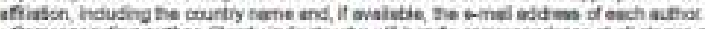

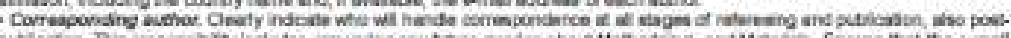

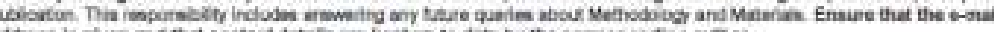

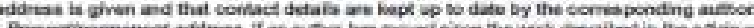

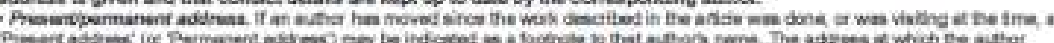

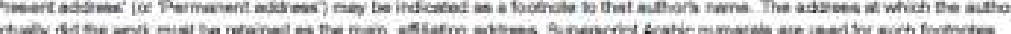

Abatract

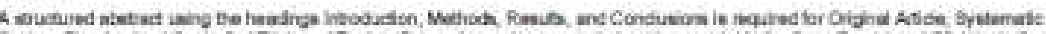

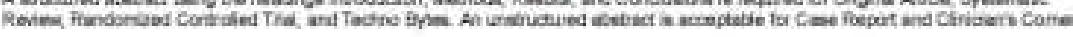

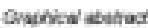

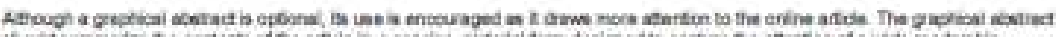

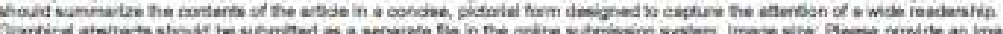

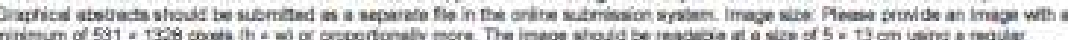

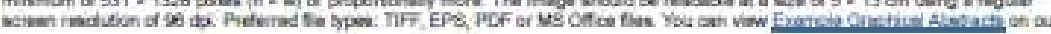




\section{ANNEX C - AJO-DO manuscript submission guidelines}

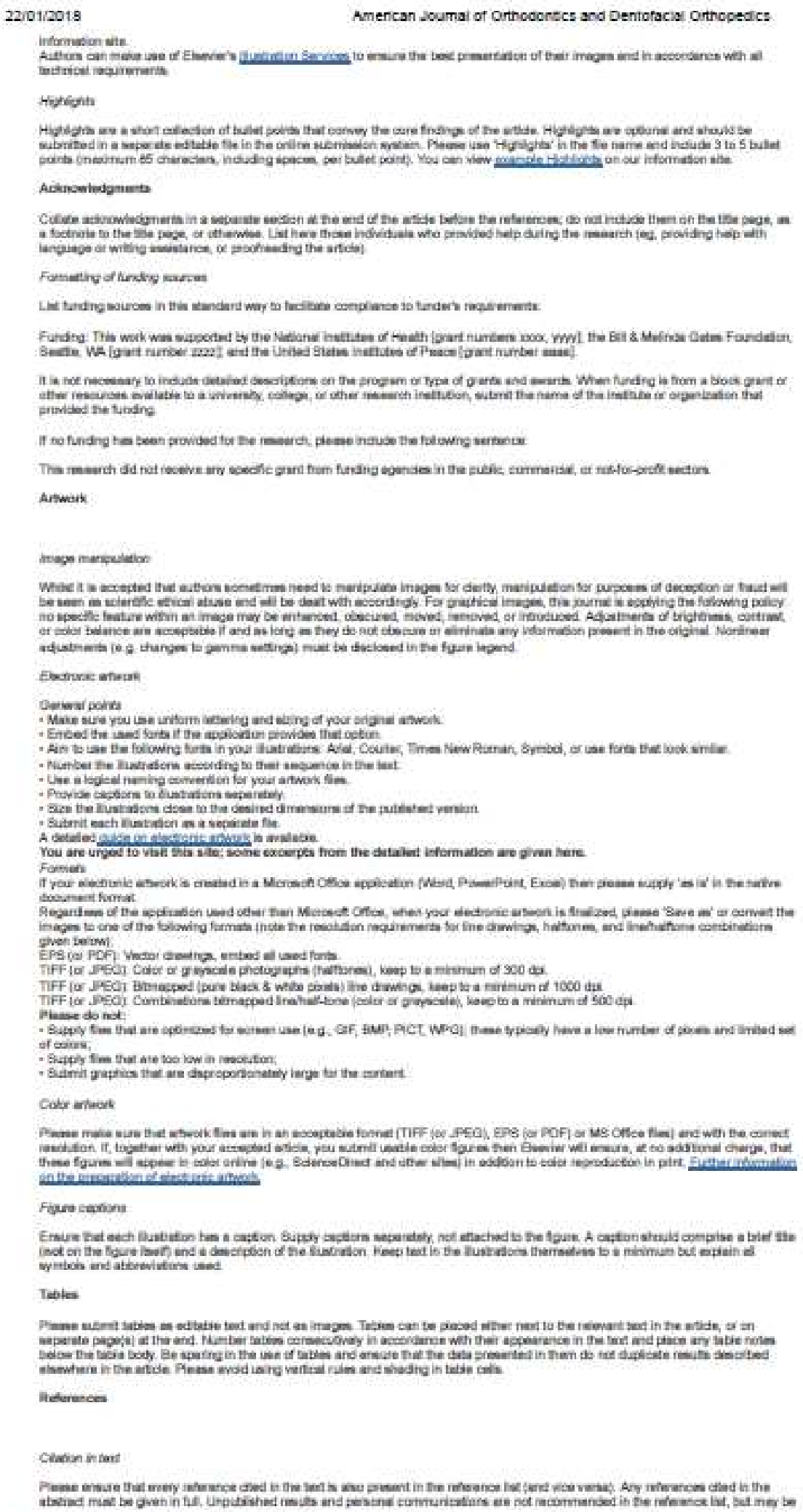

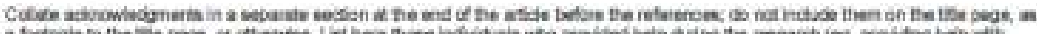

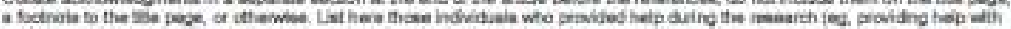

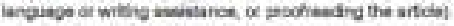

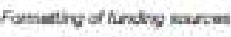

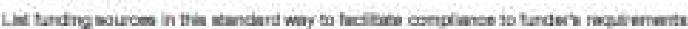

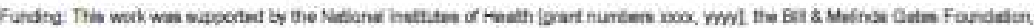

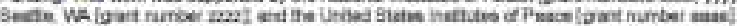

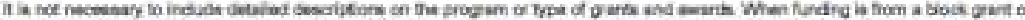

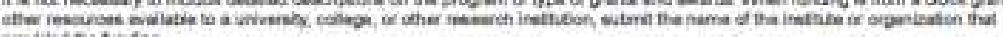
arimided the luibanc

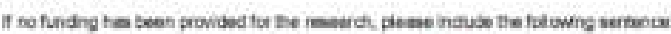

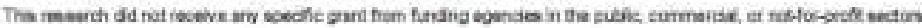

Answas

itroge maracielion

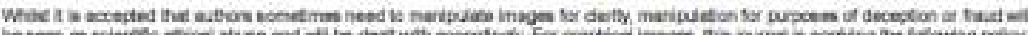

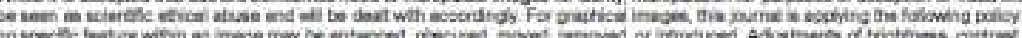

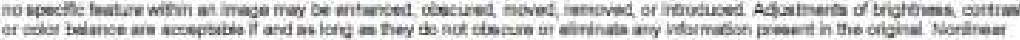

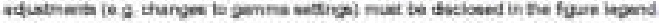

Eactroxe aherak

Oenwar poins:

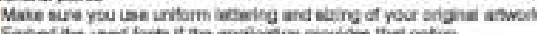

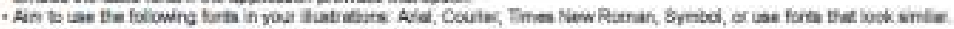

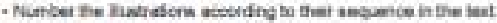

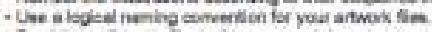

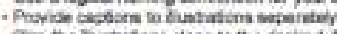

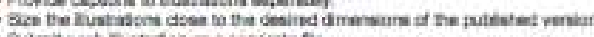

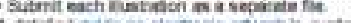

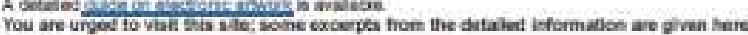

Fomen

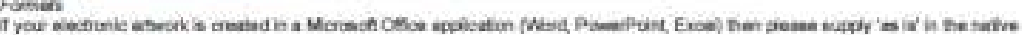
cosenent fomat

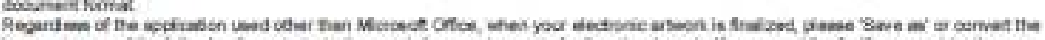

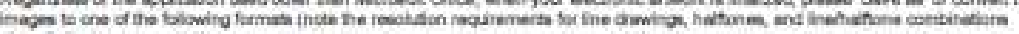

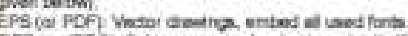

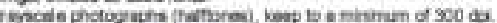

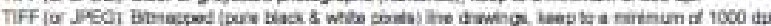

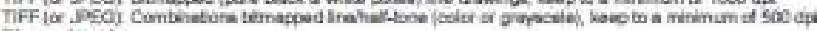

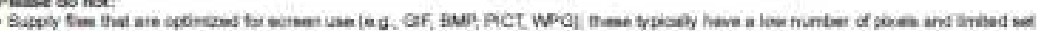

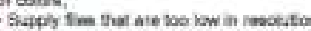

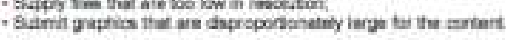

Coks mothock

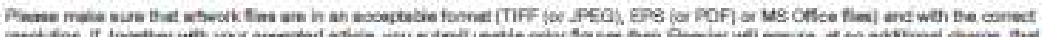

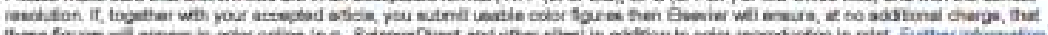

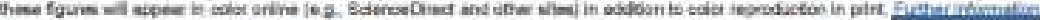

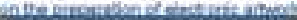

Figuresanons

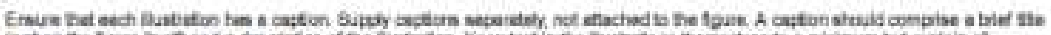

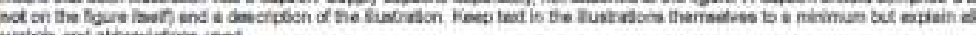

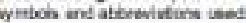

Tables

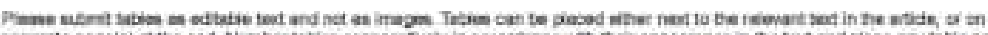

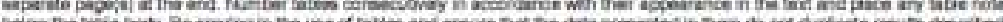

Heteresen

Ceasion iniant

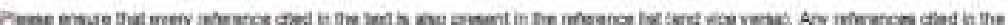

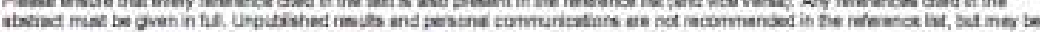




\section{ANNEX C - AJO-DO manuscript submission guidelines}

$22 / 01 / 2018$

American Joumal of Orthodontcs and Dentofacias Orthopedce

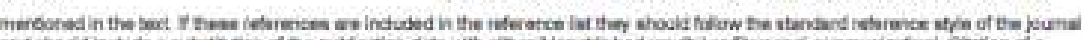

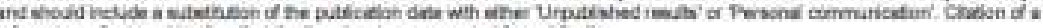

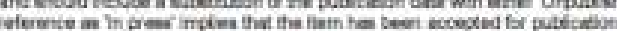

Frotorenca inks

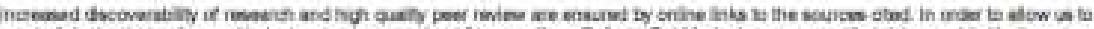

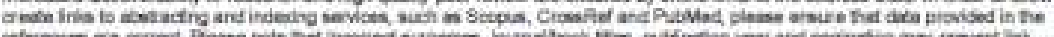

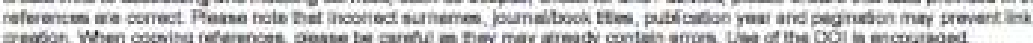

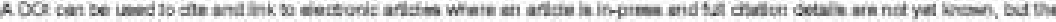

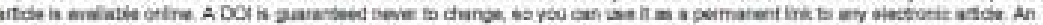

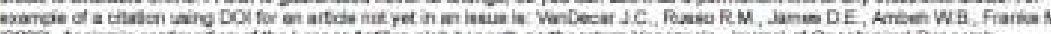

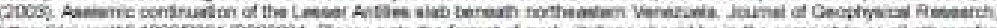

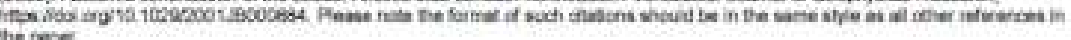
he pese

was netarenes

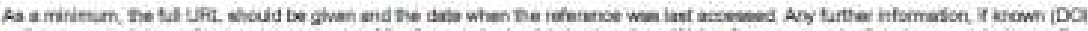

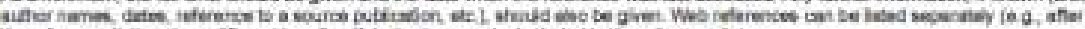

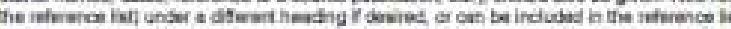

Dra intormos

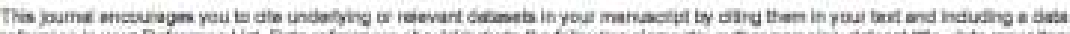

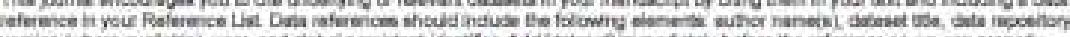

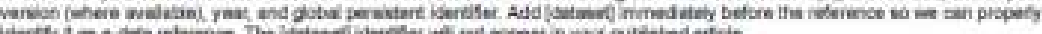

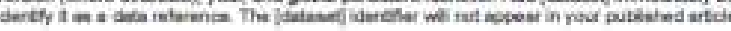

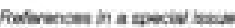

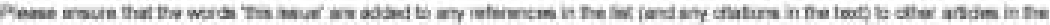
satre Soesia lowin

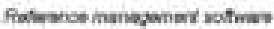

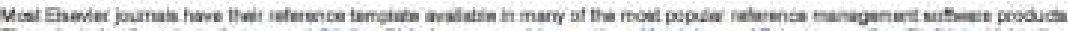

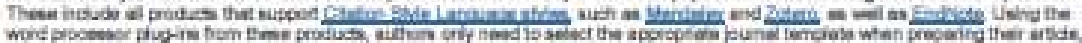

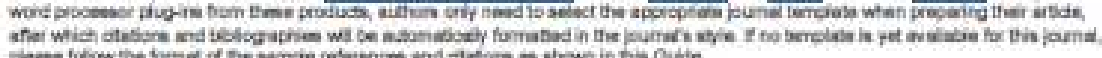

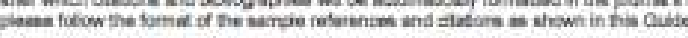

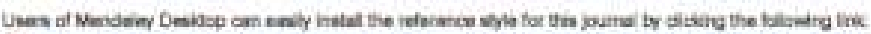

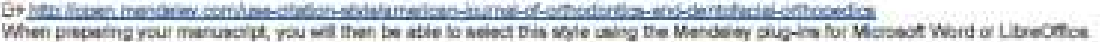
Then piopating

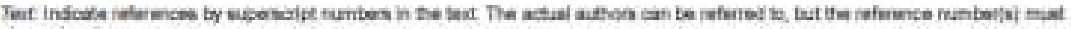
alwers be gives

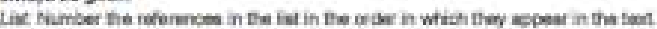

Eserpos:

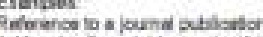

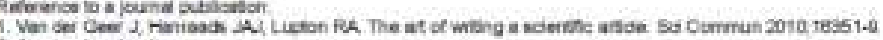

tilliratica to it boek

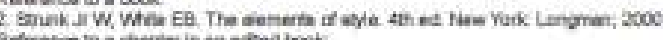

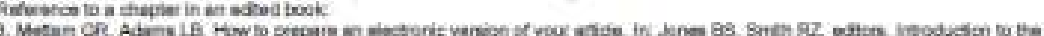

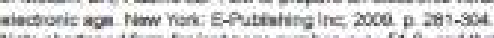

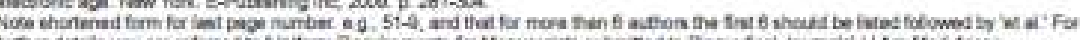

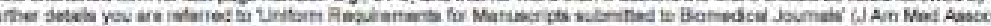

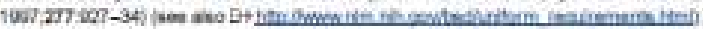

Video

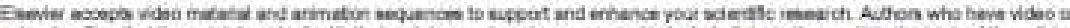

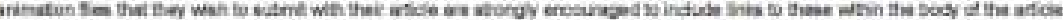

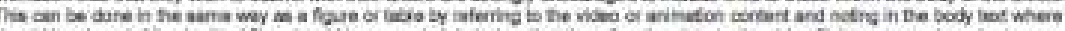

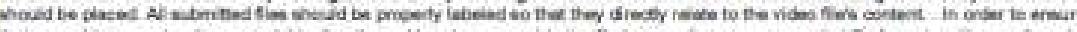

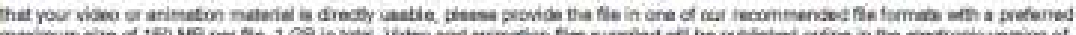

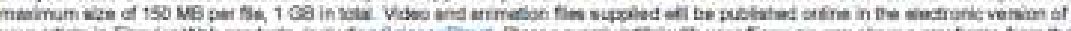

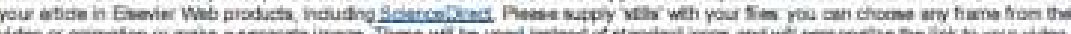

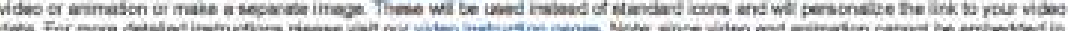

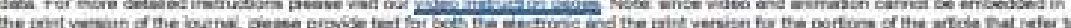
thesemars

MESEATOCHDATA

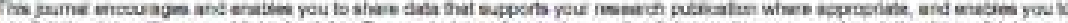

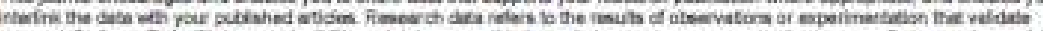

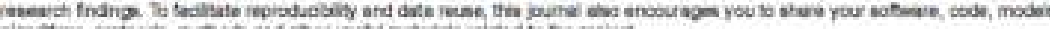

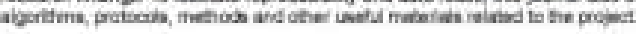

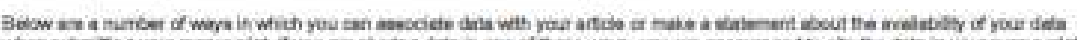

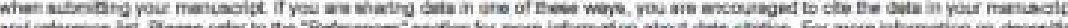

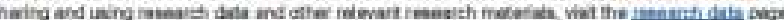

Deabuen

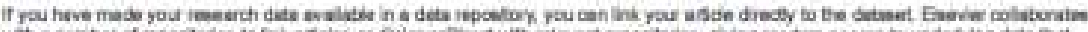

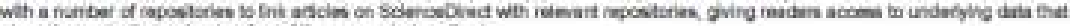

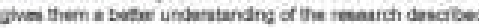

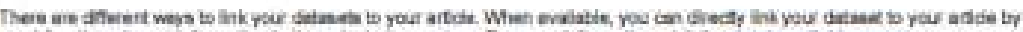

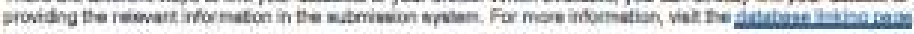




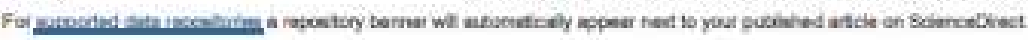

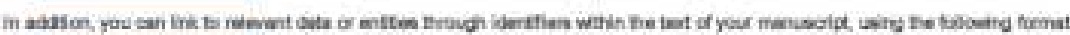

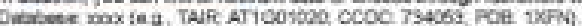

Mendisy Des:

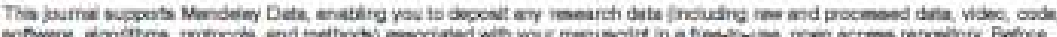

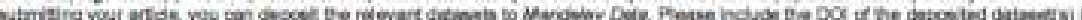

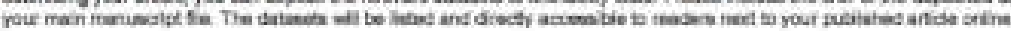

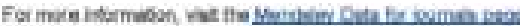

Davathomese

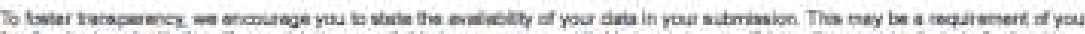

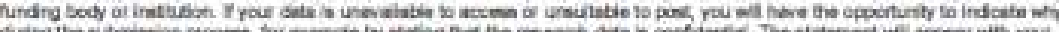

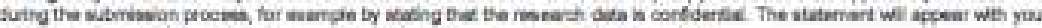

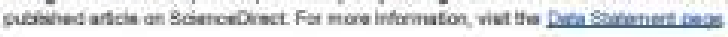

ARTCLE ENRECINETS

Autrostise

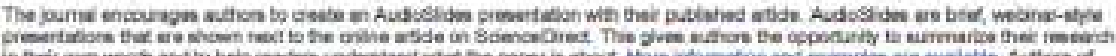

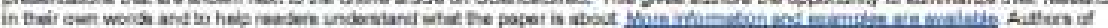

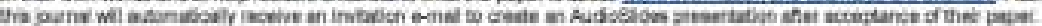

aD mode

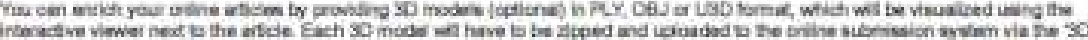

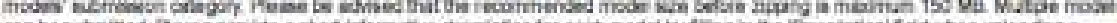

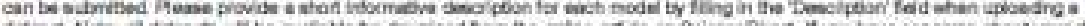

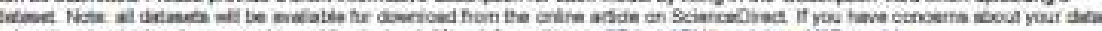

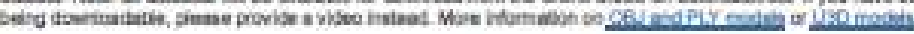

\section{Sulminsion Ctectitat}

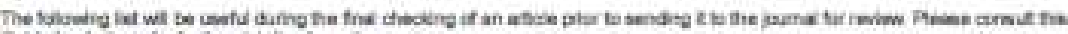

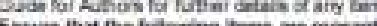

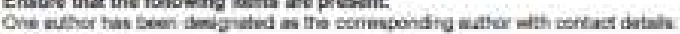

- E-Tial adians

- fil posis matron

- Postis rimters

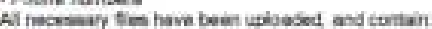

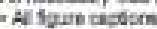

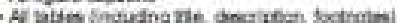

Fumer insterathis

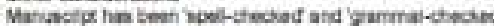

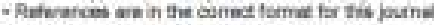

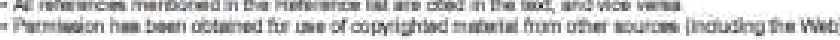

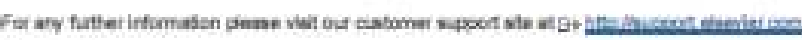

Perniabiona

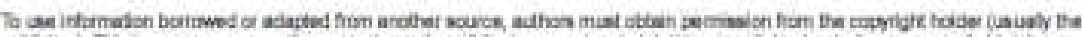

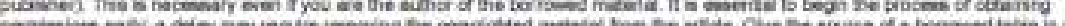

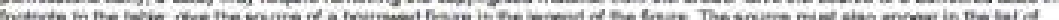

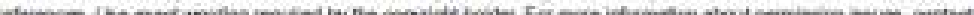

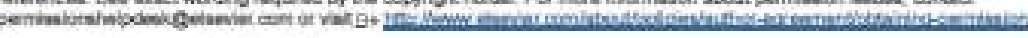

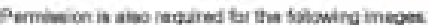

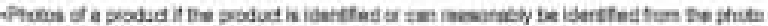

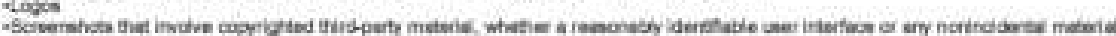

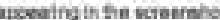

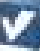

After Acceptante

Proch

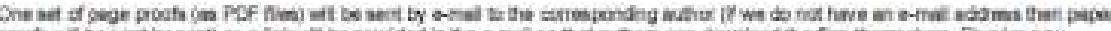

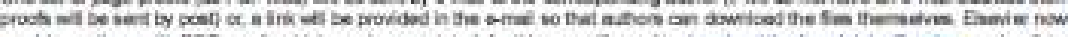

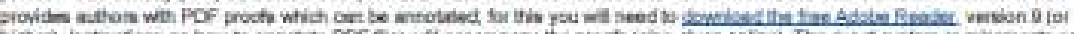

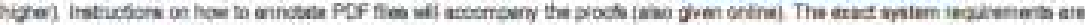

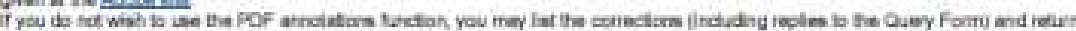

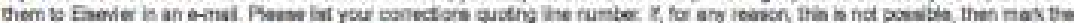

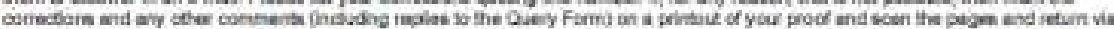

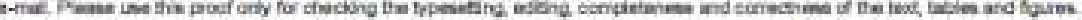

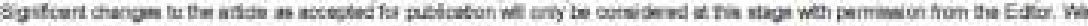

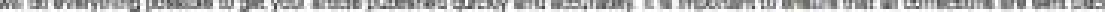

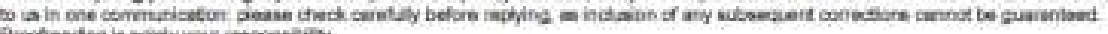

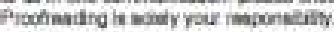

ceprints 


\section{ANNEX C - AJO-DO manuscript submission guidelines}

$$
22 / 01 / 2018
$$$$
\text { American Joumai of Orthodontcs and Dentofaclas Orthopedce }
$$

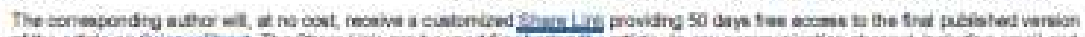

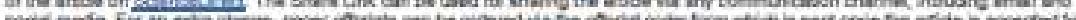

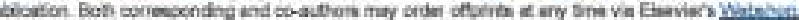

\section{Author inquiries}

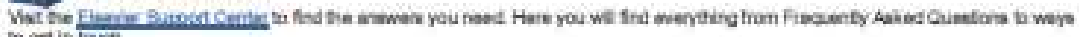

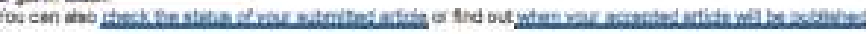

